\title{
STRUCTURAL DESIGN OF A HIGH ENERGY PARTICLE DETECTOR USING LIQUID SCINTILLATOR
}

\author{
A THESIS \\ SUBMITTED TO THE FACULTY OF THE GRADUATE SCHOOL \\ OF THE UNIVERSITY OF MINNESOTA \\ BY
}

TIMOTHY JOHN BERG

IN PARTIAL FULFILLMENT OF THE REQUIREMENTS

FOR THE DEGREE OF

MASTER OF SCIENCE

FEB, 1997 


\section{ACKNOWLEDGMENTS}

The author would like to thank all of his colleagues in the MINOS collaboration for their help in preparing this thesis. The institutes involved in the MINOS collaboration include Argonne National Laboratory, Boston College, Caltech, Columbia University, Dubna (Russia), Fermilab, IHEP-Beijing, Indiana University, ITEP-Moscow, Lebedev, Lawrence Livermore National Laboratory, University of Minnesota, PNPI-St. Petersburg (Russia), Oak Ridge National Laboratory, Oxford University (England), Rutherford Laboratory (England), Stanford University, Sussex (England), Texas A\&M, University of TexasAustin, Tuffs University, and Western Washington.

A special thank you to the physicists and staff in the University of Minnesota Physics Department involved with the MINOS project for all their help and expertise with the ongoing design of the liquid scintillator detector. Those involved in the project include P. Border, H. Courant, P. Cushman, K. Heller, D. Maxam, W. Miller, J. Nelson, E. Peterson, K. Ruddick, R. Rusack, and M. Schub.

In addition, the author would like to thank Professor Thomas Chase for his guidance and all the help he has given during the preparation of this thesis. His input was greatly appreciated.

Last, but not least, the author would like to thank his wife, Jean Giebenhain, for being very supportive during the preparation of this thesis. Without her help this thesis might never have been finished. 


\section{ABSTRACT}

This thesis presents a design for a 10,000 ton liquid scintillator neutrino detector being considered for the MINOS project at Fermilab. Details of designing, manufacturing, and assembling the active detector components are presented. The detector consists of 1080 magnetized steel absorber planes alternating with 1080 active detector planes. Each active plane is made up of plastic extrusions divided into nearly 400 cells for positional resolution. Life tests on the plastic extrusions determine their feasibility for containing the scintillator. The extrusions are sealed at the bottom, filled with liquid scintillator, and have an optical fiber running the entire length of each cell. The fibers terminate at the top of each extrusion in a manifold. An optical-fiber-light-guide connects the fibers in each manifold to a photo-detector. The photo-detector converts the light signals from the scintillator and optical fibers into electrical impulses for computer analysis. 


\section{TABLE OF CONTENTS}

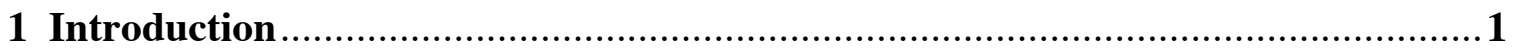

1.1 Background Of High Energy Physics Particle Detectors ...................................... 1

1.1.1 Standard Model Of Physics ....................................................................... 1

1.1.2 Motivation For Neutrino Research ……................................................... 3

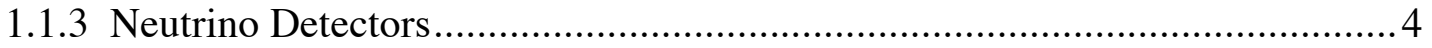

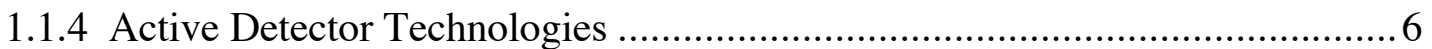

1.2 Literature Review Of Particle Detectors ............................................................ 10

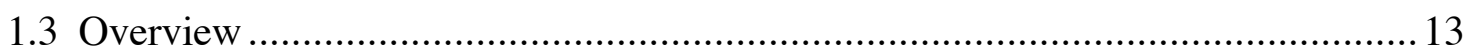

2 Requirements For A Liquid Scintillator Detector .........................................15

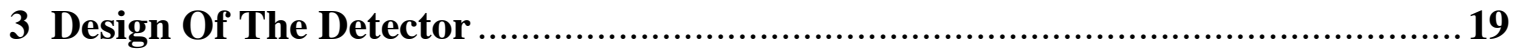

3.1 Design Of A Liquid Scintillator Detector System................................................ 19

3.2 Layout Of The Active Detector Plane …………..........................................25

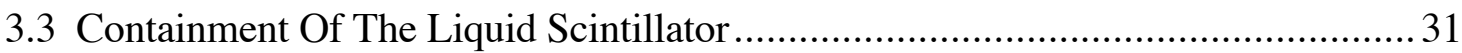

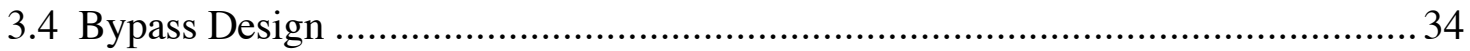

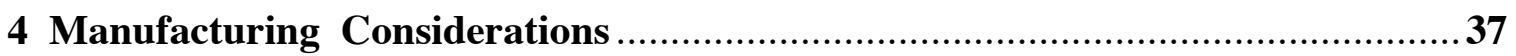

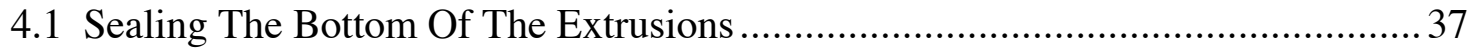

4.1.1 Endcap And Mold Design............................................................................... 37

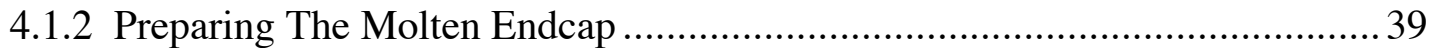

4.1.3 Applying The Endcap To The Extrusion..................................................... 41

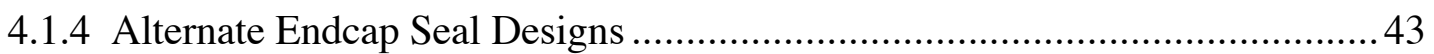

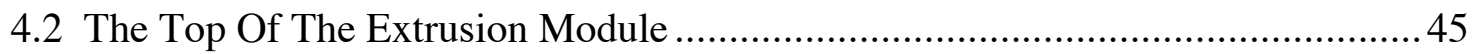

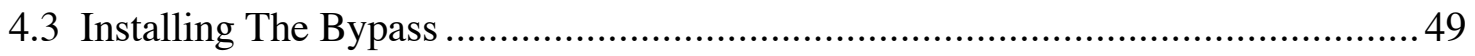

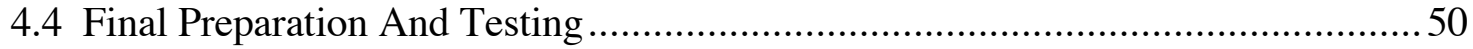




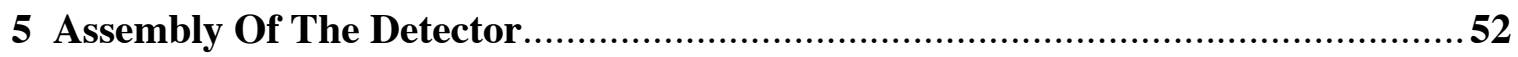

5.1 Transporting To The Detector Site And Storage ..............................................52

5.2 Assembly And Support Of The Active Detector Plane.........................................54

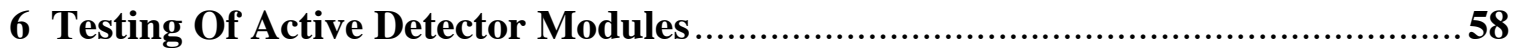

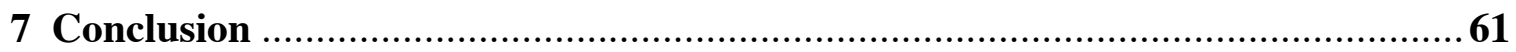

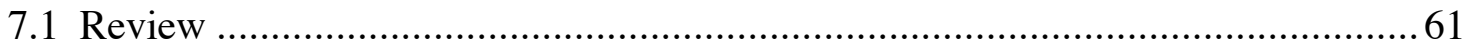

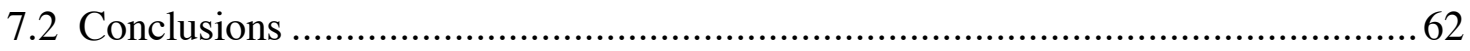

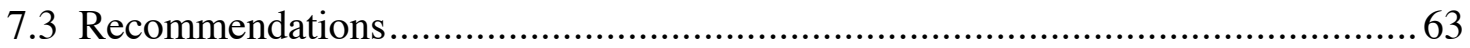

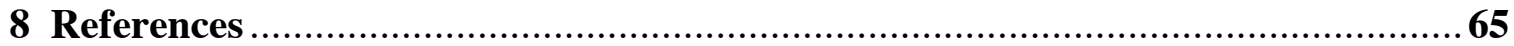

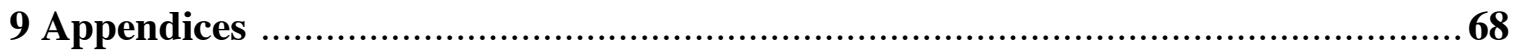

9.1 Appendix A Calculations For Components And Layout.....................................68

9.1.1 Calculate Number Of Cells .................................................................. 70

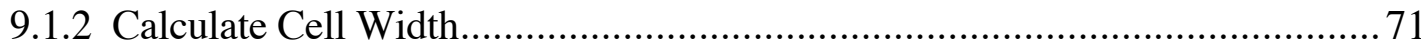

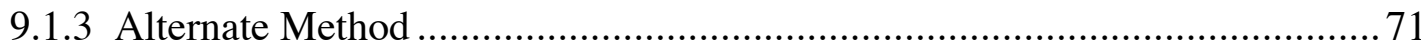

9.2 Appendix B Calculation -- Dimensions, Area, Volume, and Mass ..................... 72

9.2.1 Steel Octagon Area, Volume, And Mass........................................................ 73

9.2.2 Extrusion Area, Volume, And Mass ............................................................ 74

9.2.3 Optical Fiber--Volume And Mass (Within Octagon Area)........................... 74

9.2.4 Scintillator Volume And Mass ..................................................................... 75

9.2.5 Calculations For Total Detector.............................................................. 76

9.3 Appendix C Calculations Of Loads And Pressures.............................................. 77

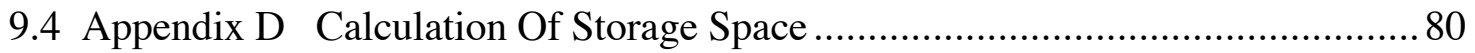

9.5 Appendix E Costing Of Scintillator Detector .................................................. 82 


\section{LIST OF FIGURES}

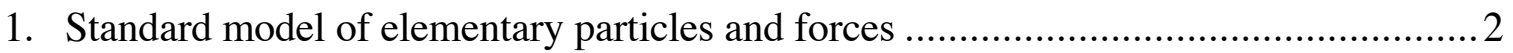

2. Illustration of how a neutrino detector is placed underground to trace the path of the particles created from neutrino interactions ..........................................................

3. Simplified version of a particle detector. A particle passing through two active detector planes has its location marked by the signals from two photo-detectors from

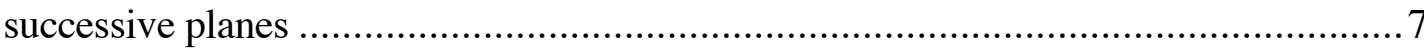

4. Perspective view of a 10,000 metric ton detector hanging on a steel support structure. Only about $15 \%$ of the steel support structure is shown ..........................................20

5. Layout of Extrusion Modules in Active Detector Plane ...........................................22

6. One corner of an octagon detector showing extrusion modules, a light guide harness attached to six alternating planes, and one photo-detector attached to a bracket on

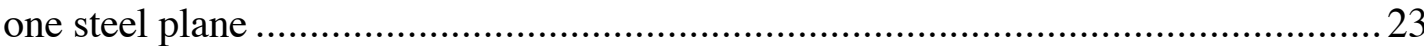

7. Simplified block diagram showing identification of particle in detector .....................24

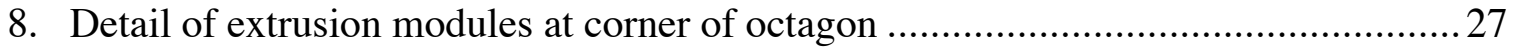

9. Layout of one active plane using 14 extrusions versus one using 12 extrusions. Note that two extrusions bypass the center hole........................................................29

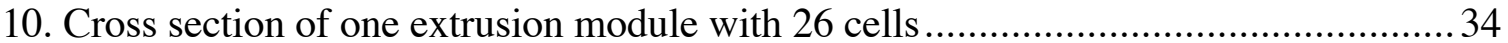

11. View of how the bypass fits into the extrusion and routes the fibers around the center

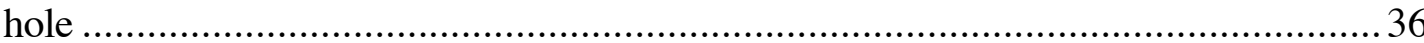

12. Close-up view of one area of the bypass showing how the fibers transition from the

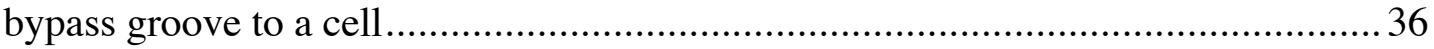

13. The endcap mold and endcap with the aluminum foil release material. ......................39

14. View of endcap applier jig and the extrusion on a table ........................................42

15. Conical seal on the bottom of the module to seal around fibers................................45

16. Motorized fiber inserter pushing fibers into an extrusion module ..............................46

17. Manifold attached to the end of the extrusion showing the fibers and cover ..............48

18. The bypass is shown below the extrusion module and fibers in preparation for the fibers to be inserted into the bypass. The bypass is then inserted into the extrusion and welded around the perimeter

19. Floorplan of mine with 3 assembly stations. A two weeks supply of steel and modules are shown but only with a one week supply of scintillator in barrels. The steel octagon planes are assembled on steel I-beams called strongbacks.... 
20. Top view of steel plane on strongback showing the support shelf and layout of extrusion modules. The shortest module is installed first against the shelf followed successively by the remainder.

21. Close up view of fill ports and vent ports on two types of manifolds

22. Graph of expansion of polypropylene extrusion from 40 psi internal pressure over a seven week period.

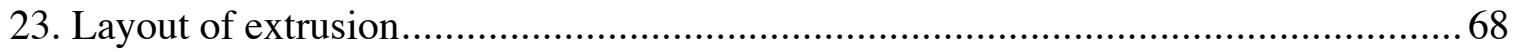

24. Corner of octagon with module and support shelf ................................................. 70

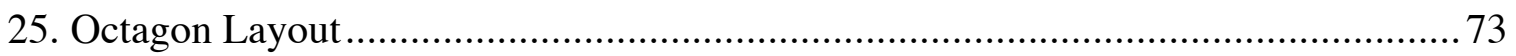

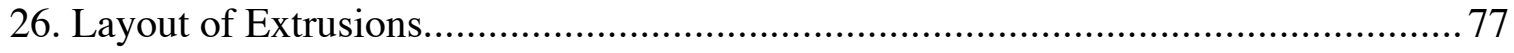

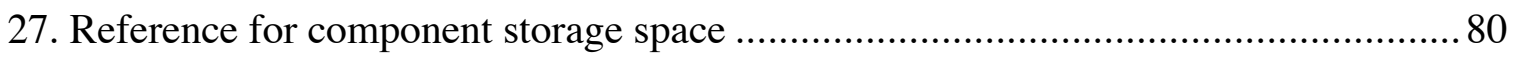

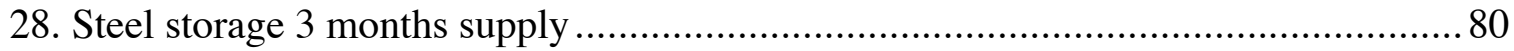

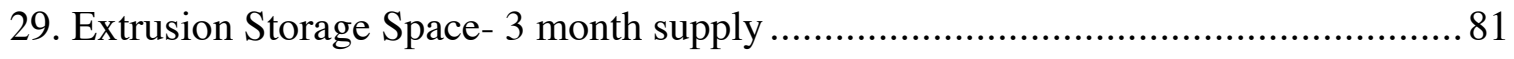

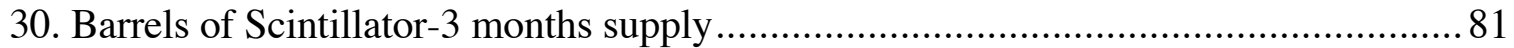




\section{LIST OF TABLES}

Table 1. List of requirements for a typical liquid scintillator detector......

Table 2. Results from internal pressure tests of a polypropylene extrusion from 40 psi pressure over a seven week period.

Table 3. Ratios of the number of extrusions across ' $L$ ' and total number of extrusions across ' $\mathrm{W}$ '. The best choices have the smallest deltas and 'dLs'.

Table 4. Costing summary of a liquid scintillator detector. 82

Table 5. Costing summary of a detector based on Iarocci tubes or RPCs..... 83 


\section{INTRODUCTION}

The first section of the introduction presents background of high energy particle detectors as a foundation for the design of new particle detectors. This includes a brief explanation of the underlying physics. A literature search discusses additional sources of information about different types of particle detectors. The final section of the introduction presents an overview of a new detector design.

\subsection{BACKGROUND OF HIGH ENERGY PHYSICS PARTICLE DETECTORS}

The background for high energy particle detectors is broken down into four parts. The first section briefly explains the Standard Model of Elementary Particles. The Standard Model relates the smallest subatomic particles and the forces that bind these particles together. The second section focuses on neutrino research. Neutrinos are one class of subatomic particles defined in the Standard Model. The third section looks at the two basic components that compose neutrino detectors. Most of the detector is made of a dense material such as lead or steel. The other component is the active detector. The final part of this section discusses different active detector technologies.

\subsubsection{STANDARD MODEL OF ELEMENTARY PARTICLES AND FORCES}

High Energy Physics is the science of the study of subatomic particles. We are all familiar with particles like protons, electrons, and neutrons. However, protons and neutrons are made of still smaller particles.

The Standard Model of Elementary Particles and Forces in Fig. 1 (Perkins, 1987) describes elementary particles of matter called quarks and leptons as well as force carriers 
called bosons. Quarks come in six types. The first generation of quarks, the up and down, make up protons and neutrons. Four other types were present at the beginning of the universe and can now be created in particle collisions using high energy accelerators.

The six leptons include the familiar electron and two other particles called the muon and the tau. Each of these three particles is associated with one-and only one-of three neutral leptons called neutrinos. The electron pairs with the electron neutrino, the muon with the muon neutrino, and the tau with the tau neutrino.

Force carriers called bosons round out the Standard Model. The four bosons consist of the photon which transmits electromagnetic force, the gluon that transmits the strong force that hold the quarks together in the nucleus, and the $\mathrm{W}$ and $\mathrm{Z}$ bosons that transmit the weak force associated with radioactive decay.

A detailed review of the Standard Model is given by Barnett, et al., (1996).

\section{ELEMENTARY PARTICLES}
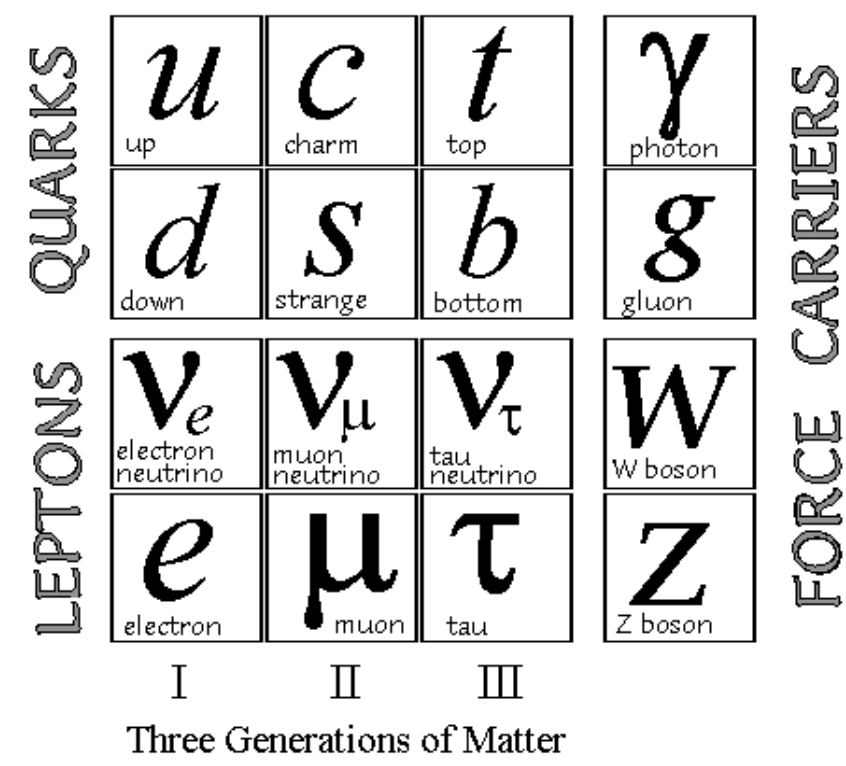

Figure 1. Standard model of elementary particles and forces 


\subsubsection{MOTIVATION FOR NEUTRINO RESEARCH}

The existence of neutrinos was first postulated by physicist Wolfgang Pauli in a 1930 unpublished letter (Pontecorvo, 1982) to explain the apparent loss of energy during radioactive decay. If such a particle did exist, Pauli theorized, it would have almost no mass, no charge and would virtually have no interaction with other particles. Such a particle would be nearly impossible to detect.

If neutrinos did exist, nuclear reactors, developed in the 1950's, should produce a large number of neutrinos. Frederick Reines and Clyde Cowan (Reines, 1994) became the first physicists to detect neutrinos from a nuclear reactor in South Carolina, proving their existence.

As methods of detecting neutrinos improved, experimenters could count the number of neutrinos from different types of experiments. Experimenters in a mine in South Dakota in the 1960's (Davis, 1994) were able to count the number of electron neutrinos that arrived from the sun. However, the number counted was only half of the number of electron neutrinos expected. Other experiments counting muon neutrinos (Cardall and Fuller, 1996) also showed a deficit including the experiment at Soudan 2 (Gallagher, 1996). What happened to the rest? Could a mystery that $90 \%$ of the universe appears to be missing or unseen, so-called dark matter (Reines, 1994), also figure into the puzzle?

The Standard Model suggests that neutrinos are massless particles that rarely interact with other particles. What if this were not so? What if neutrinos were not massless (Shrock, 1996) but instead, have some minute mass? Then, neutrinos would be able to oscillate. Oscillation is defined as changing from one state or type to another and back again (Reines, 1994). Only particles that have mass can oscillate from one type to another. Some of the past detectors could only count one type of neutrino and therefore, missed 
the neutrinos that may have oscillated to another type. This could account for the missing neutrinos from past experiments. In addition, the question of dark matter in the universe could be partially resolved if neutrinos have mass.

\subsubsection{NEUTRINO DETECTORS}

This section describes the basic design and location of a current generation of neutrino detectors.

Abandoned mines provide an excellent environment for the location of neutrino detectors. The earth acts as a shield, blocking out most of the cosmic radiation which creates noise in neutrino detectors. Neutrinos rarely interact with matter so these particles have no trouble passing right through the earth and out the other side.

Currently, neutrino detectors are being designed to look for neutrinos that oscillate from one type to another. For example, a high energy beam of protons can be aimed at a far away detector (Fermilab, 1996). A target is placed in the path of the beam near the beam's origin. Protons hit the target and fragment into subatomic particles. In turn, some of these subatomic particles will decay into muons and muon neutrinos. All the particles will gradually be absorbed or stopped by the earth until only muon neutrinos remain to continue the journey. Finding evidence of other types of neutrinos in the detector besides muon neutrinos will prove that neutrinos can oscillate and therefore have mass - however minute.

Interaction of neutrinos with other particles is a very rare event and interaction of oscillating neutrinos with other particles would be extremely rare. New detectors are being designed to search for these extremely rare interactions (Gomez-Cadenas and Gonzalez-Garcia, 1996). The chances of detecting such an interaction improve with large 
massive detectors - the more mass the better. Neutrinos can interact with any type of mass so massive detectors use dense materials to keep its size as small as possible.

The principal material in a detector is called the absorber. Some detectors use lead for the absorber due to its high density. However, the absorber in many detectors is made of steel because steel is cheaper than lead and it can also be used for structural support.

If the detector were only made up of the absorber, neutrino particle interactions, also called hits, could not be detected. Active detector layers or planes placed perpendicular to the path of moving particles must alternate with absorber layers to trace a particle's path resulting from a neutrino interaction. Each type of particle associated with each type of neutrino has its own signature path. Figure 2 shows the alternating steel and active detector layers.

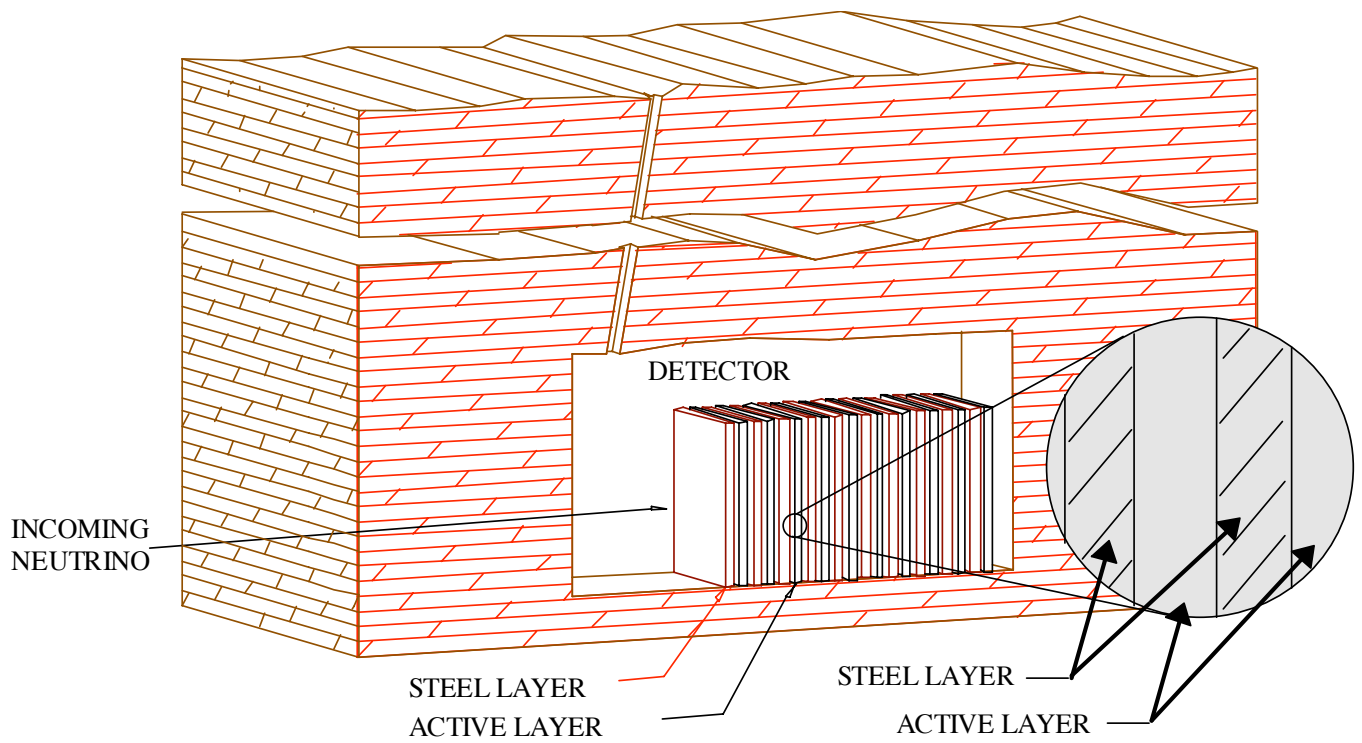

Figure 2. Illustration of how a neutrino detector is placed underground to trace the path of the particles created from neutrino interactions.

For proper particle identification, the position of the particle must be traced from plane to plane to determine its path in the z-direction. Depending on the type of active detector, 
each plane may give the position of the passing particle for either one or two axes. If only one axis is determined per active plane, then two active detector planes oriented perpendicular to each other are required to locate the particle on an $x-y$ coordinate system.

\subsubsection{ACTIVE DETECTOR TECHNOLOGIES}

The active detector elements come from a variety of technologies. These currently include Iarocci Tubes ${ }^{1}$, resistive plate capacitors (RPCs), and Scintillator technologies using solid scintillator or liquid scintillator.

The theory of Iarocci Tubes and RPCs will be briefly explained before concentrating on Scintillator technology for the rest of this section. The theory of using scintillator for positional resolution is discussed. The advantages and disadvantages of scintillator are compared to the other technologies. Then, the focus is turned to what can be done to reduce or eliminate the disadvantages of liquid scintillator while enhancing the advantages.

An active detector plane made of Iarocci Tubes has an electrically grounded plane on one side of the active plane. A cross sectional view of the active plane shows the plane divided into a row of rectangular tubes or cells. Running down the middle of each cell is a charged wire. Each cell acts as a capacitor that, when a particle passes through, discharges. The discharge creates an electrical signal which is read out by a computer that tracks the particle through the detector.

RPCs are similar to Iarocci Tubes in that they use a discharge to signal a particle's passage. RPCs do not have a wire in the middle of each tube. Rather, both sides of the active detector layer of the RPCs act as the capacitor.

\footnotetext{
${ }^{1}$ Iarocci Tubes are also known as limited streamer tubes.
} 
Scintillator technology relies on a transparent scintillating material that emits photons when energized by particles passing through the material. The photons are sensed by a photodiode or another type of photo-detector such as a photomultiplier tube (PMT) (Reines, 1994). The photo-detector converts light energy to an electrical signal.

Scintillators have been utilized since the 1910's in a variety of experiments including the discovery of the nucleus in 1911. Scintillator was also used in the discovery of the neutrino (Reines, 1994). Recently, scintillator with optical fibers have been proposed to provide better position resolution in detectors at lower cost.

The theory of scintillator design for position detection is simple to understand. An active detector layer consists of several rectangular tubes or cells arranged in a row that cover one entire plane. Each cell runs the entire width or height of the plane. Arranging the cells to be perpendicular in adjacent planes provides both $\mathrm{x}$-axis and $\mathrm{y}$-axis position information. Figure 3 shows one corner of a octagon shaped particle detector and how the position of a particle is determined by two planes of rectangular cells.

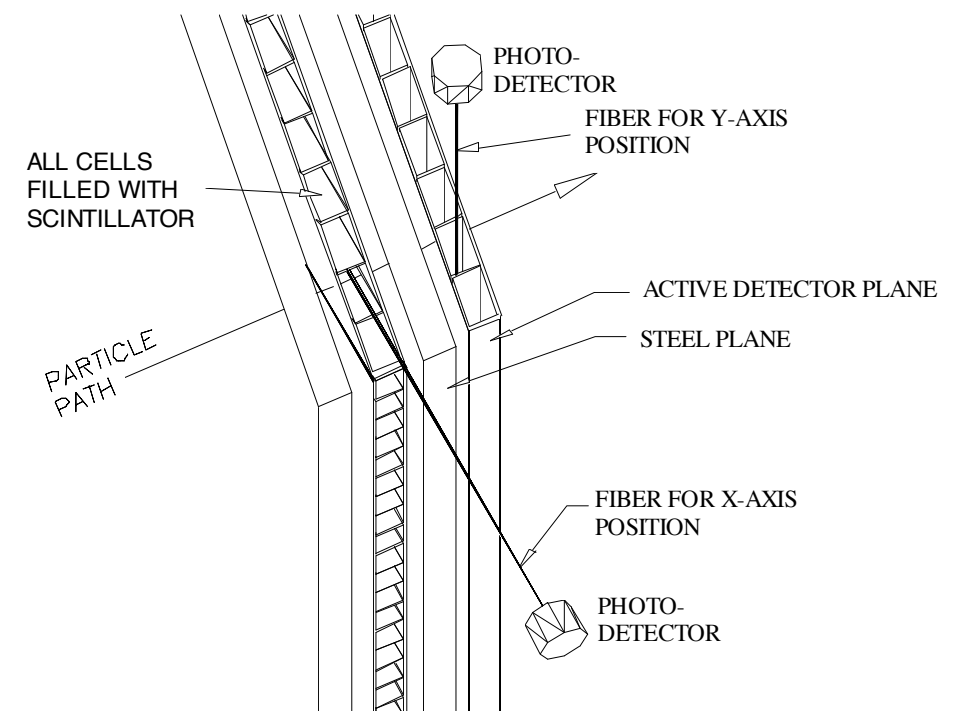

Figure 3. Simplified version of a particle detector. A particle passing through two active detector planes has its location marked by the signals from two photodetectors from successive planes. 
Filling each cell is a scintillating material or scintillator. The scintillator may be either liquid or solid. Solid scintillator is integrated with a clear solid plastic binder. Liquid scintillator is mixed with a liquid such as mineral oil. The scintillator contains chemicals that transforms the energy from a particle passing through the scintillator into photons. The amount of photons produced is proportional to the number of passing particles. Two particles produce twice the number of photons.

The photons produced by the scintillator bounce off the reflective cell walls until the light is captured inside an optical fiber. The optical fiber runs the entire length of the cell. The light is transported by the fiber to the outside of the detector.

The light signal from the fiber is picked up by one channel of a photo-detector that transforms the photons into an electrical signal. The strength of the electrical signal is related to number of particles passing through the cell. Therefore, the number of passing particles can be counted by the strength of the electrical signal.

Scintillator systems have many advantages over Iarocci Tubes and RPCs. Scintillator systems can distinguish between two particle interactions that are very close together, also called nearby hits, making it easier to count the number of interactions. Iarocci tubes and RPCs cannot distinguish between nearby hits. Scintillator systems also have faster timing than the alternatives making them able to accommodate faster processing of information.

One disadvantage of scintillator systems is cost. While the design of a liquid scintillator detector and a solid plastic scintillator detector is similar, liquid scintillator is half as costly as solid scintillator of similar resolution. Even so, the liquid scintillator design is still more expensive than competing technologies. 
Another major disadvantage of liquid scintillator systems is their tendency to leak. Many current liquid scintillator detectors have metal tubes with photo-detectors at both the top and bottom of the tubes. However, the hydrostatic pressure from the liquid scintillator sometimes causes the scintillator to leak from the tube's bottom fittings.

Leaks from the detector can be a concern. If the scintillator is toxic, leakage may have health consequences. The scintillator may give off fumes requiring greater ventilation. Even if the scintillator is benign, replacing and cleaning up lost scintillator is problematic.

The focus of this thesis is to take advantage of the strengths of the previous liquid scintillator detectors and to minimize or eliminate the disadvantages while reducing the cost of the scintillator system. The challenge is to reduce the costs of this design while maintaining the integrity of the structure and improving the response of the system.

Solving the leakage problem of liquid scintillator systems would eliminate most disadvantages of the system. Recent improvements in light collection, due to improved optical fibers and reflectivity of the cell walls, may make this possible. Higher light collection enables reducing the number of photo-detectors by half by eliminating photodetectors from the bottom of the tubes. The bottom of the tubes can then be completely sealed, thus reducing if not eliminating the leaking problem. An added benefit is significant cost savings from fewer photo-detectors.

Costs can be reduced further by changing the containment material from metal to plastic. Several tubes can be combined into one multi-cell plastic extrusion. Furthermore, reflective materials can be added to plastic to increase light yield. Metal tubes would need to be painted to obtain achieve equivalent reflectivity. 
By far, the most expensive component of the liquid scintillator system is the photodetector. While this is an area that needs to be scrutinized, reducing the costs of individual photo-detectors is an independent issue not covered here. Reducing the costs of photo-detectors will be done in parallel with reducing the costs of the rest of the detector components, but only the latter will be addressed within the scope of this thesis.

\subsection{LITERATURE REVIEW OF PARTICLE DETECTORS}

The articles in this literature review cover two topics. First, all types of scintillator detectors are presented including current liquid scintillator detectors searching for neutrino oscillations. Also presented are detectors other than scintillator detectors searching for neutrino oscillations.

The discovery of the neutrino was made over forty years ago by Frederick Reines and Clyde Cowan (Reines, 1994). The neutrino was discovered with liquid scintillator detectors using nuclear reactors in Hanford, Washington and along the Savannah River in South Carolina as a likely source of neutrinos.

The detector consisted of containers of liquid scintillator with photomultiplier tubes (PMT) attached to the ends of the containers. The liquid scintillator's role was two-fold. First, the scintillator transformed the energy from a neutrino particle interaction into light photons. Then the scintillator acted as a medium to transport the light photons to the PMT attached to the containers. The PMT transformed the light photons into electrical signals.

The length of each container in Reines and Cowan's detector was short because the light from the particle interaction attenuated quickly as it traveled in the liquid scintillator. The attenuation was from the lack of clarity in the scintillator. In the detector described in this 
thesis, the light travels inside an optical fiber with greater clarity than liquid scintillator. In addition, the fiber shifts the wavelength of the light, further reducing attenuation.

In the 1960's, the Homestake Gold Mine detector (Davis, 1994) was built to detect electron neutrinos emanating from the sun. The first results showed less electron neutrinos than what was expected from calculations. The results have not varied much since. This experiment, in addition to others, fueled the notion that neutrinos may have mass and may oscillate from one type to another. Detectors like the one presented in this thesis are being designed to look for these neutrino oscillations. Additional sources for the Homestake experiment are Beier (1995) and Nakumura (1996).

The Large-Volume Detector (LVD) (Pless et al., 1993; Aglietta et al., 1995) is located at the Gran Sasso Laboratory in Italy. One mission is to search for atmospheric neutrino oscillations. The LVD is made of a combination of liquid scintillator and limited streamer tubes. The source of neutrinos for this experiment is from cosmic rays. By comparison, the source of neutrinos for the detector presented in this thesis is from an accelerator beam.

KARMEN (Zeitnitz, 1994; Drexlin, 1994) is another detector that searches for neutrino oscillations using liquid scintillator. KARMEN contains 56 tons of high resolution liquid scintillator. The containers of liquid scintillator are optically isolated from each other. Attached to the end of each container is two PMTs. Again, the liquid scintillator is used as the transport medium for the photons to reach the PMTs as in Reines and Cowan's detector. Additional sources for the KARMEN detector are Cardall and Fuller (1996) and on the World Wide Web (KARMEN collaboration , 1996).

The CHORUS and NOMAD detectors (Gomez-Cadenas and Gonzalez-Garcia, 1996) are located at the CERN Laboratory in France. Both detectors search for neutrino oscillations 
and other events but use different technologies not presented in this thesis. The CHORUS detector utilizes both an emulsion and a scintillating fiber section. The NOMAD detector is essentially an electronic bubble chamber. Both detectors use beams from accelerators.

Some think the future success of finding neutrino oscillations from these detectors is small. The potential success of the detector presented in this thesis could be larger because it will scan a larger range of oscillation-parameter space. Other sources for information on the NOMAD and CHORUS detectors are Patzak (1995), Laveder (1995), and on the World Wide Web (CHORUS collaboration, 1996; NOMAD collaboration, 1996).

The final currently operating detector presented in this section is the Liquid Scintillator Neutrino Detector (LSND) at Los Alamos National Laboratory (Gomez-Cadenas and Gonzalez-Garcia, 1996). The LSND is a liquid scintillator detector that searches for neutrino oscillations. At the present time, a handful of events at the LSND may possibly be the result of neutrino oscillations but none been confirmed (Shrock, 1996). The design of the LSND is essentially an 8 meter tank filled with liquid scintillator with photodetectors installed around the inside perimeter of the tank. The LSND operates from an accelerator beam like the design presented in this thesis. Additional sources for the LSND can be found on the World Wide Web (LSND collaboration, 1996).

At Fermilab, at least two new experiments (Gomez-Cadenas and Gonzalez-Garcia, 1996) are being designed to search for neutrino oscillations in addition to other events. The first is COSMOS, a short baseline experiment meaning the location of the detector is relatively close to the accelerator beam source. COSMOS is similar to the CHORUS detector. The second is the MINOS experiment.

MINOS is a long baseline experiment with a large detector placed far from the accelerator beam source. The unique design of MINOS, in addition to the far away detector, is a 
smaller detector placed close to the accelerator beam. The results from the larger, far detector will be compared to the results from the smaller, near detector. MINOS could have a better chance of detecting neutrino oscillations than either NOMAD or CHORUS.

The design of the detector presented in this thesis is one of several proposed for the MINOS far detector. The author was a part of the MINOS collaboration during the writing of this thesis. Additional information on MINOS and COSMOS can be found on the World Wide Web (MINOS collaboration, 1996; COSMOS collaboration, 1996).

Most of the references presented in this section present the topic with an audience of physicists in mind. Even though the information may be hard to understand for those who are not physicists, some useful information can be gleaned from the references for the purposes of this thesis. The World Wide Web sites are particularly helpful to nonphysicists.

\subsection{OVERVIEW}

This thesis presents a design for a liquid scintillator system that eliminates the disadvantages of past scintillator systems. Requirements for a workable liquid scintillator system are formally defined in Chapter 2.

Chapter 3 discusses the physical size and shape of a typical liquid scintillator detector fulfilling the requirements defined in Chapter 2. A block diagram of the detector's process for particle identification is presented. Details of all the component designs are developed. Most importantly, a containment vessel for a liquid scintillator plane that does not leak over the estimated $10-15$ year life of the detector is required.

The containment vessel is made from plastic extrusions that are attached to the absorber plane. The design and layout of the active detector plane optimizes the number of 
extrusions and the number of cells in each extrusion based on factors such as detector plane width and geometry and the number of channels per photo-detector. The extrusions on each plane are arranged to satisfy the 2-D coordinate system required to determine positional information of a particle passing through the detector.

Chapter 4 discusses the manufacturing process of extrusion modules. An extrusion module, or module for short, is defined as an assembled and completely sealed unit containing an extrusion, an endcap, a manifold, and fibers. The bottom of each extrusion is sealed using a newly developed thermo-forming method of melting an endcap to the extrusion end. A manifold used for filling liquid scintillator and routing fibers is attached to the top of each extrusion. Two center extrusion modules also require a bypass to circumvent a hole through the length of the detector.

Assembly techniques are discussed in chapter 5. Quality assurance testing is performed on the modules before they are assembled in the detector. The modules are pressure checked to determines if any leaks exist. The fibers in the modules are optically checked for continuity and clarity.

After the quality tests have been passed, the modules are attached to the steel plane and supported by a steel shelf. The modules support one another and are contained horizontally between the steel planes.

Chapter 6 presents life testing. Life testing approximates the performance of extrusions as they are subjected to internal hydrostatic pressures and external loads from other modules over a period of time. The parameters of the modules such as pressure and temperature are intentionally exaggerated to provide accelerated results. The testing determines what type of support is required for the modules. 


\section{REQUIREMENTS FOR A LIQUID SCINTILLATOR DETECTOR}

Any workable liquid scintillator detector capable of observing interactions between particles involving different types of neutrinos must satisfy many requirements. The detector will be a massive structure - on the order of 1000-10,000 metric tons or more. The containment vessel for the liquid scintillator is designed to be structurally sound to eliminate leaks. The containment vessel must be divided into many cells of less than $4 \mathrm{~cm}$ width per plane for proper position resolution. Each cell must have an optical fiber that runs the entire length of the cell. The cell walls must have reflectivity of greater than $95 \%$ to ensure enough light is collected by the fiber. The fibers will exit only from the top of each cell. All the fibers must terminate at a photo-detector which transforms the light signal into an electrical signal. All the components of the detector must be sized to be manageable and transportable and yet compatible with large absorber planes. The final challenge is to accomplish all these goals while minimizing the cost of the detector.

Table 1 lists the overall requirements for a liquid scintillator detector. Each requirement is summarized by means of a metric, units associated with that metric and limiting values. The remainder of this section describes selected metrics from this table in greater detail.

The structure of the containment vessel is dictated by the $8 \mathrm{~m}$ height and 10 year life requirements. The vessel will need to withstand the hydrostatic pressures developed in a large detector having a height of 8 meters. The material used for the containment vessel must not deteriorate from the effects of the liquid scintillator. The material chosen should be strong enough to support the load from the scintillator and cells above the lowest cell once installed between the absorber planes. 
The temperature requirement of less than $40^{\circ} \mathrm{C}$ results from the requirement to magnetize the detector. In some detectors using a steel absorber, the steel is magnetized by an electrical current in a copper conductor running through the center of the detector. Heat will be generated by the electrical current. The material in the containment vessel must be able to withstand the increase in temperature.

\begin{tabular}{|l|l|l|}
\hline \multicolumn{1}{|c|}{ METRIC } & \multicolumn{1}{|c|}{ UNIT } & \multicolumn{1}{c|}{ VALUE } \\
\hline Total Mass & $\mathrm{kg}$ & $>1,000,000$ \\
Cell width & $\mathrm{cm}$ & $<10,000,000$ \\
Reflectivity & $\%$ & $>95$ \\
Operating Life & years & $>10$ \\
Height & $\mathrm{m}$ & 8.0 \\
Type & reference & magnetized \\
Maximum Operating Temperature & ${ }^{\circ} \mathrm{C}$ & $<40$ \\
Module width & $\mathrm{m}$ & $<2$ \\
Number of extrusions & reference & even \\
Photo-detector Channels & number & max. available \\
Cost & $\$$ US & $<50 \mathrm{M}$ \\
\hline
\end{tabular}

Table 1. List of requirements for a typical liquid scintillator detector.

To keep costs low, a number of cells in the containment vessel can be joined together into one extrusion. The width of all extrusions should be the same. All extrusions should contain the same number of cells.

The absolute maximum width of an extrusion is determined by the size of the entrance to the detector location. Neutrino detectors are usually placed underground to shield them 
from most cosmic radiation. Abandoned mines provide deep underground space at comparatively low cost. Thus, all detector components must fit through the entrance shaft.

The maximum width restriction listed in Table 1 is based on the Soudan mine in northern Minnesota. The maximum dimension of the shaft is 2 meters. Other mines may have different width restriction. The maximum width of an extrusion may also be limited by the manufacturing limit of the extruder.

If the detector is magnetized, one plane of active detectors contains an even number of extrusions symmetrically placed around the center hole. In this case, an odd number of extrusions cannot be used because the center extrusion would accommodate the entire center hole. The center extrusion could never be replaced once the magnetic coil is installed. Furthermore, accommodating the center hole by means of two extrusions per plane with peripheral hemispherical indentations simplifies overall manufacture and installation of the extrusions.

The extrusion modules may be built off-site because of space limitations underground. If so, the modules will be transported from a factory to the underground site. The modules need to be completely self-contained for protection. The fragile optical fibers must not extend from the modules where they could be damaged. The material for the modules should withstand minor impacts.

Once the modules are inside the hall where the detector is being assembled, the active detector system is integrated with the absorber. The active detector planes alternate with the absorber planes. The absorber planes will be directly supported by a support structure. The detector planes can be attached to separate support structures or directly to the absorber planes. Attaching the active planes to the absorber planes reduces costs by eliminating additional support structures. Provision for attaching the photo-detectors, 
optical harness, and associated electronic equipment and wiring needed to complete the system are made through brackets attached to the absorber planes.

The cost of the photo-detectors is nearly proportional to the number of photo-detectors regardless of the number of channels in each. The cost is about the same for 64 channels as it is for 156 channels. Thus, the number of channels in a photo-detector should be maximized.

The cost of a detector should be minimized while still satisfying its basic physics requirements. In any case, the cost of the detector should not exceed $\$ 50$ million to make funding the experiment feasible. 


\section{DESIGN OF THE DETECTOR}

The design of a typical liquid scintillator detector is presented in Section 3.1 of this chapter. The design evolves from the requirements set forth in Chapter 2. Certain assumptions must be chosen regarding size parameters. Among these are the total mass, steel thickness, and active detector thickness.

The design of the rest of the detector is determined once the parameters are chosen. Section 3.1 presents different methods for the support of the detector. The layout of the detector is also discussed. The layout affects the cell size, the required number of photodetectors, and the connections for the fibers to the photo-detectors.

This chapter includes three additional sections. Section 3.2 elaborates on the method for determining the layout of the active detector plane. Section 3.3 details the choice for the containment vessel material. Finally, Section 3.4 discusses the method of detecting from those cells cut in half by the center hole of a magnetized detector.

\subsection{DESIGN OF A LIQUID SCINTILLATOR DETECTOR SYSTEM}

A large liquid scintillator detector, based on the MINOS detector shown in Fig. 4, will have a mass of 10,000 metric tons ( 22 million pounds). The steel absorber planes account for $90 \%$ of the detector's mass. The detector will be magnetized by an electrical coil passing through the center of the detector. The diameter of the center coil hole is $30 \mathrm{~cm}^{1}$.

The magnetic field generated by the current flowing through the coil will be circular in direction about the center hole axis. Based on magnetic field lines, the optimum cross-

\footnotetext{
${ }^{1}$ The size of the hole is a function of amount of electrical conductor needed for the magnet coil and whether the conductor will need to be water cooled.
} 
section for the detector is circular. However, an octagon shape is adopted to reduce $\operatorname{costs}^{2}$. The width and height of the octagon are chosen to be 8 meters, satisfying a requirement for the detector.

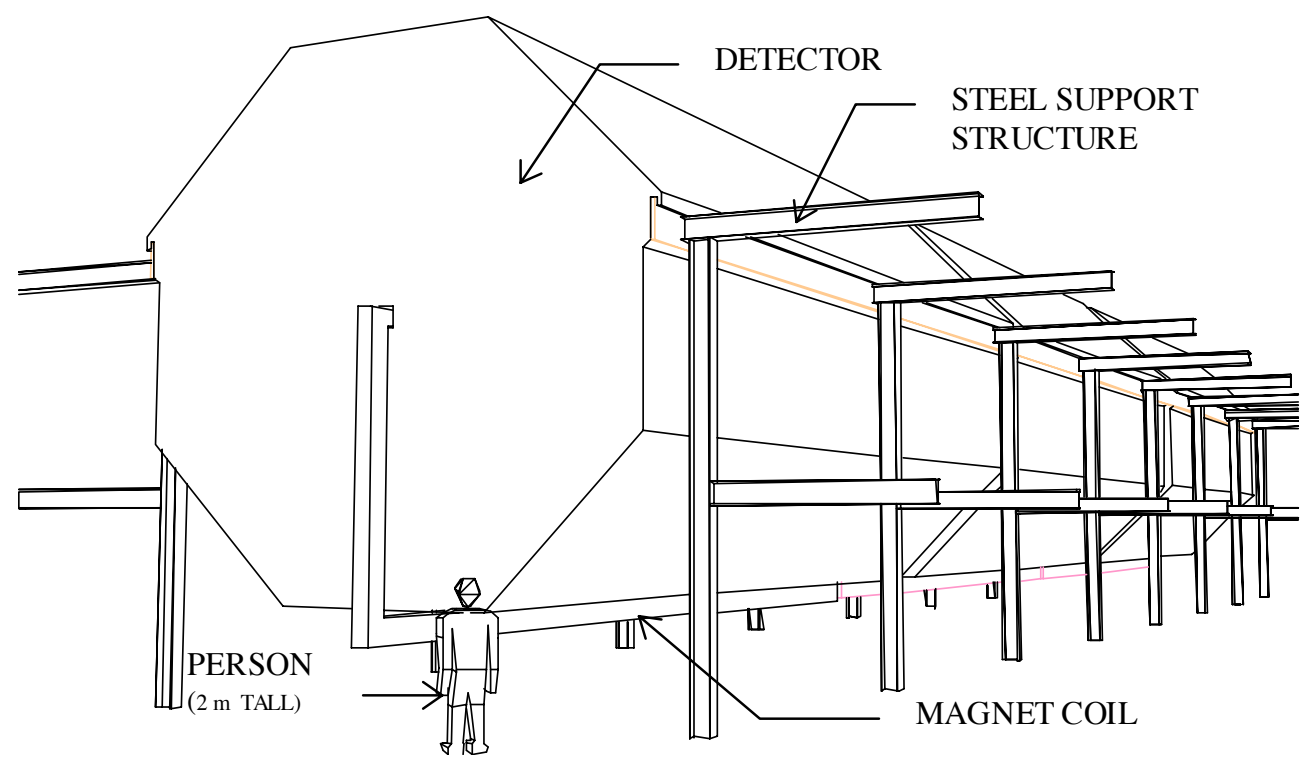

Figure 4. Perspective view of a 10,000 metric ton detector hanging on a steel support structure. Only about $15 \%$ of the steel support structure is shown.

The length of the detector is a factor of the thickness and number of the absorber planes and the active detector planes. Thinner steel planes and active planes allow a particle from a neutrino interaction to penetrate more layers for the same path length. The result is better resolution. Therefore, the thickness of the steel planes and the thickness of active detector planes are chosen to be nominally $2 \mathrm{~cm}$ each. Based on the density of the steel and the density of the scintillator and plastic, 1080 planes will be required.

Since it is impossible to make a steel plane completely flat, a space of $1.0 \mathrm{~cm}$ is added between the steel planes for the active detector modules. It is acceptable in the liquid scintillator design to allow a certain amount of crushing to the active plane to minimize the

\footnotetext{
${ }^{2}$ The octagonal shape is dictated by the steel design, which is not addressed here. However, the octagonal shape adapts well to mounting the liquid scintillator modules, as discussed in Chapter 5.
} 
free space between the steel planes. The resulting approximate detector length is 54 meters. The detector is divided into three sections for easier installation of the coil. The total required length is 60 meters allowing for 1 meter clearance between each section and 2 meters on each end.

The aspect ratio of each vertical plane is similar to the cardboard backing on a writing tablet. The planes may be susceptible to buckling but lateral support is given by bolting the successive planes of steel together. The planes support each other. Space for each active detector plane is maintained by the bolt spacing.

Additional support for the detector steel planes can be supplied in many ways. The simplest and easier way to support the detector is to mount the steel planes on two long plates of steel reminiscent of wide railroad rails. A second support system option is to hang the steel planes from the ceiling or a steel superstructure above the steel planes. Buckling, in this case, is no longer an issue.

A third method of support hangs the steel planes from two rails on each vertical side of the octagon. Thus, the steel hangs similar to hanging file folders. This scheme allows better access to the top of the detector, provides support for walkways on each side, and also eliminates any buckling. This method is used in Fig. 4. The hanging rail support method will be assumed for the remainder of this thesis.

Figure 5 shows the layout of one active detector plane. Each plane consists of 14 extrusion modules laid out at $\pm 45^{\circ}$ from vertical. Successive planes of active detectors are perpendicular to the previous plane satisfying the requirement for two dimensional position resolution. Section 3.2 details the layout of the active detector plane. 
The extrusion modules vary in length from $4.3 \mathrm{~m}$ to $8 \mathrm{~m}$ long. Each module is sealed completely at the bottom and filled with liquid scintillator. Every module contains 26 cells or tubes that are each $2.17 \mathrm{~cm}$ wide.

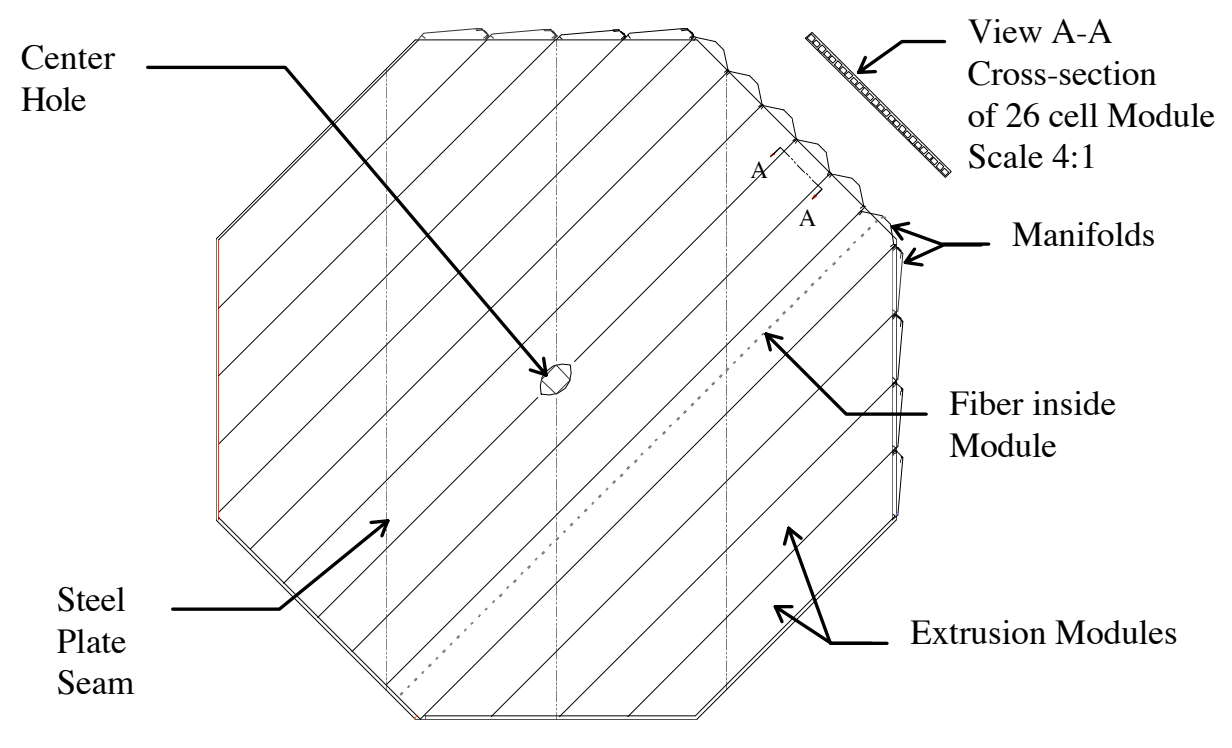

Figure 5. Layout of Extrusion Modules in Active Detector Plane

An optical fiber extends the entire length of each cell and terminates at the top of a manifold. The manifold funnels the fibers to a smaller termination area. The pathway of the fibers to the photo-detector is completed through an optical fiber harness or light guide which connects to the top of the manifold. The manifold also provides access holes for venting and filling the extrusions with liquid scintillator.

Optical fiber harness, light guide, and light guide harness are synonymous terms. A light guide is made of clear optical fibers. The light guide provides a transition from several modules to the photo-detector. The design of the light guide depends on the number of channels in a photo-detector and the number of cells in a module. 
Figure 6 illustrates how an optical light guide harness attaches to a photo-detector and six alternating active detector planes of an octagon detector. The first steel layer covering the active layer is cut-away to show the orientation of the active detector modules.

A photo-detector with 156 channels connects to one end of an optical harness ${ }^{3}$. The fibers in the optical harness are divided equally between six terminal blocks at the opposite end of the harness. Each of the terminal blocks attach to one of 6 extrusion modules, aligning the fibers in the light guide with the fibers in the manifold.

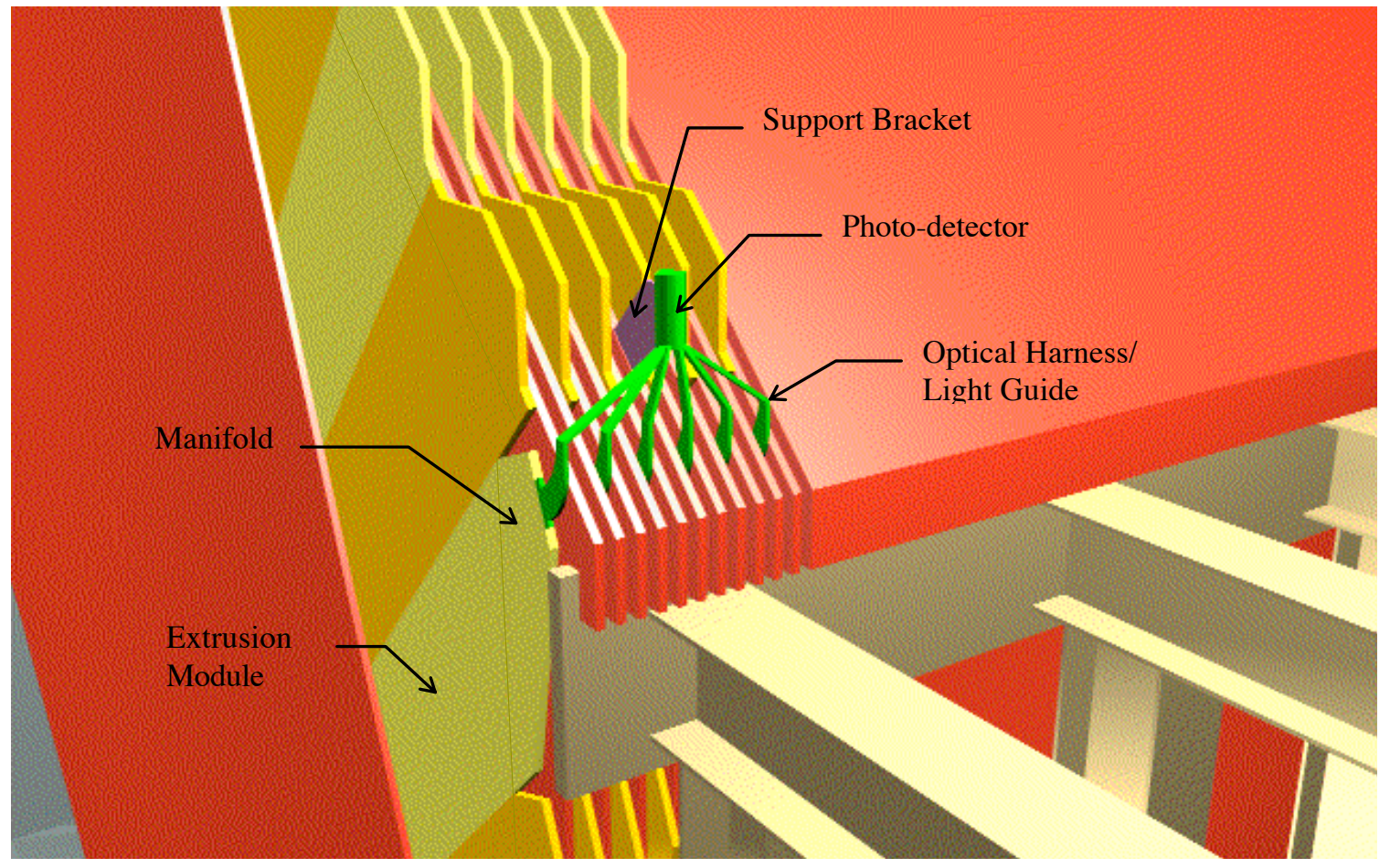

Figure 6. One corner of an octagon detector showing extrusion modules, a light guide harness attached to six alternating planes, and one photo-detector attached to a bracket on one steel plane.

To reduce the length of fibers in the harness, the light guide harness is attached to six extrusion modules from the same position in six different planes. The harness must be

\footnotetext{
${ }^{3}$ This satisfies the requirement for the maximum number of channels available in a photo-detector.
} 
attached to extrusions from alternating active planes with the same orientation. A $2 \mathrm{~cm}$ steel plane and a $2 \mathrm{~cm}$ active plane with a $1.0 \mathrm{~cm}$ gap results in $5.0 \mathrm{~cm}$ between successive active planes and $10.0 \mathrm{~cm}$ between alternating active planes. The total distance across 6 alternating active planes is $52.0 \mathrm{~cm}$.

Each channel in the photo-detector represents one specific cell in the detector. Every channel is wired to a central computer. The computer maps each cell position in the detector with software.

A wiring harness connects each photo-detector to the computer. The computer gathers electrical impulses from the photo-detectors for each neutrino interaction. Each impulse is one in a series of position inputs for each particle. All the impulses are analyzed to tell the computer the path of the particle. The particle is identified by the computer by matching the impulse data to known patterns.

Figure 7 shows a simplified block diagram of how a particle is identified by the detector using known particle paths. A moving particle energizes the scintillator in one cell creating light photons. The photons enter an optical fiber and are transported to a photodetector. The photo-detector transforms the photons to an electrical impulse. The computer gathers many impulses to construct the path of the particle. The path is compared to known particle paths and the particle is identified.

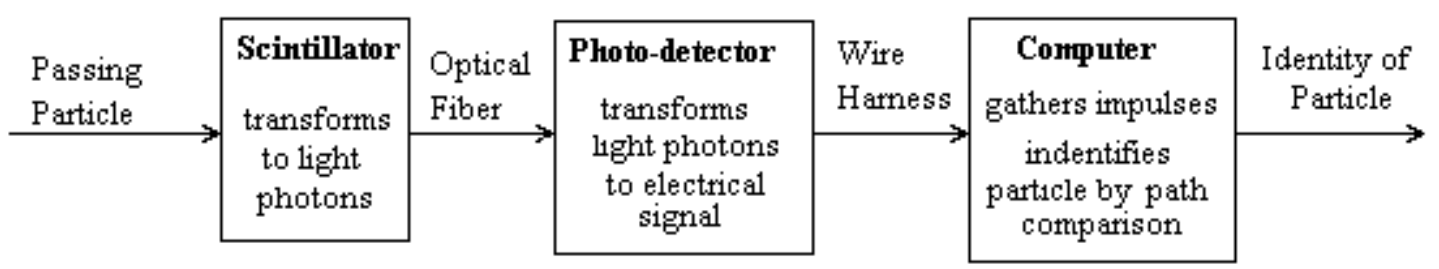

Figure 7. Simplified block diagram showing identification of particle in detector. 
A 10,000 metric ton detector can be difficult to imagine. One way to visualize the size of the detector is to express its size in terms that are familiar. The number of semi-trucks required to transport the major materials or the size of the required storage space are two such ways to express its size.

Transporting and storing the major components involves many trucks and a large amount of space. To deliver the steel for the detector would require 360 semi-trucks. This does not include the steel required for the support structure. The steel plates would cover a football field to a depth of 1 foot. The scintillator could be delivered by 32 tanker trucks like those used for gasoline delivery. Another method of transport would require 4330 barrels holding 55 gallons each. The scintillator would flood a football field to a depth of 9 inches. Thirty-four semi-trucks would be required to deliver the plastic extrusions, which would occupy about the same volume as the steel plates.

The optical fibers may not take up as much storage space as the major materials but the length of the fiber required is still impressive. The detector uses over 3 million meters of optical fiber. This translates to 1865 miles or roughly the distance from New York City to Salt Lake City, Utah.

\subsection{LAYOUT OF THE ACTIVE DETECTOR PLANE}

This section discusses how the containment vessel is divided into modules and cells. The requirements state that the cell width be less than $4 \mathrm{~cm}$ wide for positional resolution. In addition, the maximum width of a module is 2 meters. The width of the cells and modules is measured parallel to the surface of the plane. The thickness of the module is set at $2 \mathrm{~cm}$ and measured perpendicular to the plane's surface. 
Every active detector plane is made up of extrusion modules. The modules have a number of cells or tubes that are laid out in a single row all pointing in the same direction. The modules are installed parallel to one another across one plane approximating the octagonal shape of the steel absorber plane. The cells essentially divide the plane into several distinct areas. The nominal width of the cells is chosen to be approximately $2 \mathrm{~cm}$ wide.

The layout of the active detector plane is determined from a process based on the geometry of the detector, the number of channels in a photo-detector, and the nominal cell width to observe neutrino events. The smaller the cell width, the better the resolution for particle position detection. Countering this philosophy is the cost of the photodetectors. By making the cell width larger, the number of photo-detectors is reduced which significantly reduces the cost of the detector. To keep the cost of the detector at a minimum, the cell size should be as large as possible while maintaining acceptable resolution.

The design of the photo-detectors has been evolving in conjunction with the scintillator active detector. The number of channels available in a photo-detector has been consistently increasing to minimize its cost. An early design included 64 channels per photo-detector. An intermediate design increased to 96 channels. The most recent design includes 156 channels per photo-detector. As the number of channels increase, specifying the number of cells per module becomes more difficult. Therefore, a different approach is taken to determine the layout of the active detector plane.

The first step is to look at the geometry of the detector and determine the best number of extrusions. To keep handling costs down, the extrusions must be of a manageable sizenot too wide and not too narrow. The range of the number of extrusions per plane is set 
between 8 and 20. In addition, an even number of extrusion modules is required to place extrusions symmetrically around the center coil hole.

Manufacturing and assembly costs are reduced by incorporating modules that have flat ends on the bottom. The bottoms may be cut perpendicular to its length or at a $45^{\circ}$ angle depending on its location in the plane. If the bottom of one extrusion is cut to match the corner of the octagon, the base will include a discontinuity that will be difficult to seal. This type of extrusion bottom should be avoided. Figure 8 shows an example of each type of extrusion bottom in an octagon detector.

A final consideration is the support shelf required to support the extrusions on the

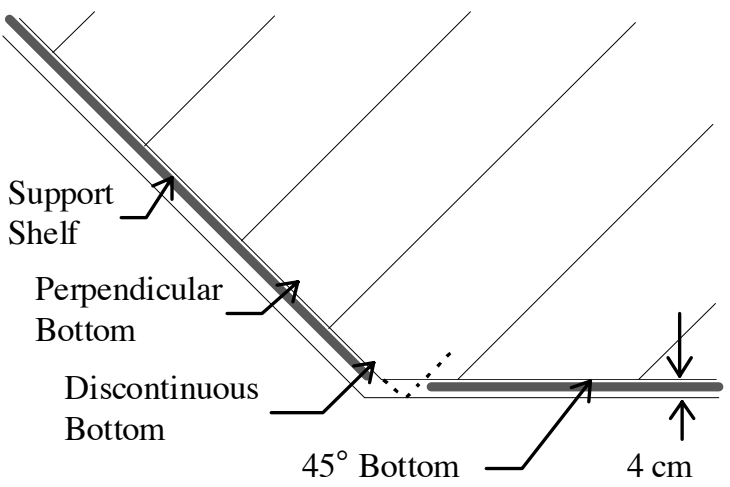
absorber plane. The support shelf is made from $2 \mathrm{~cm}$ by $2 \mathrm{~cm}$ strips of steel Figure 8. Detail of extrusion modules at corner of octagon. approximately $130 \mathrm{~cm}$ long. Eight strips are required to support an entire plane. The support shelf is positioned around the four lower edges of the detector. Figure 8 shows a portion of the support shelf at one corner of the octagon.

The support shelf reduces the overall width of the extrusions. The shelf is positioned with its inside edge $4 \mathrm{~cm}$ from the edge of the octagon. The shelf itself is $2 \mathrm{~cm}$ wide. This leaves another $2 \mathrm{~cm}$ on the outside edge for welding the shelf to the octagon. The width of the active detector plane is reduced by $4 \mathrm{~cm}$ on each side because of its symmetrical design. 
The number of extrusions should be such that no extrusion extends past the outer edge of the octagon. Using a graphical process of elimination, 14 extrusions becomes the optimal number for an octagon as shown in Fig. 9. In this configuration, only 5.7 centimeters of one extrusion overlaps a corner of the shelf. This is tolerable in this design. Comparing the dotted lines in Fig. 8 with the dimension in Fig. 9 should clarify how much the module can overlap the shelf corner. Even if the detector is set on the floor, no corner of any module will protrude beyond the edge of the absorber. Appendix A offers an analytical solution to choosing the number of extrusions per plane.

Figure 9 also shows the layout of one active detector plane using 14 extrusions compared with one using 12 extrusions. The layer with 12 extrusions includes 2 modules that extend past the edges of the octagon steel layer. This layout could be used only in a hanging method where the bottom of the detector is suspended above the floor. Only a discontinuous bottom shown in Fig. 8 would prevent the two extrusion modules from extending past the octagon edges if 12 extrusions are used.

Note that the center hole illustrated in Fig. 9 is square instead of circular. The center hole for the coil can be whatever shape is more economical if the hole is large enough for the coil installation. 


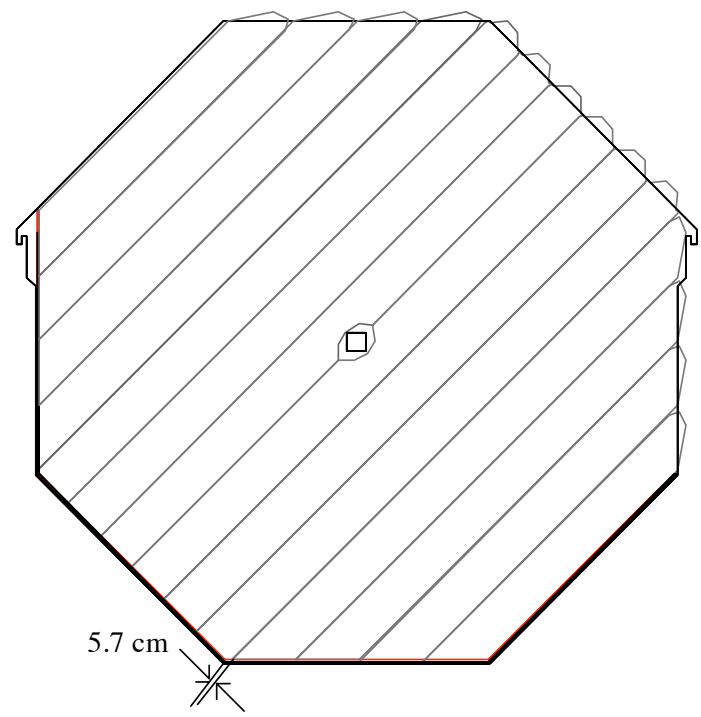

14 EXTRUSIONS

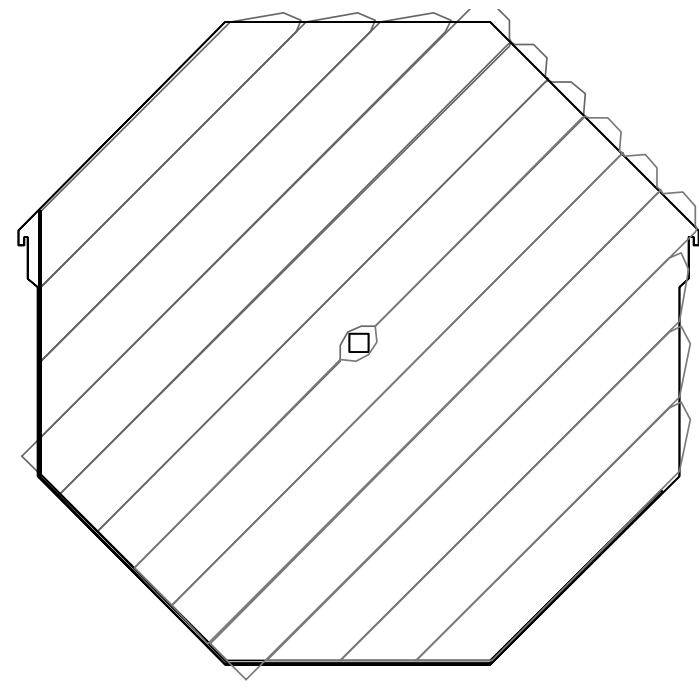

12 EXTRUSIONS

Figure 9. Layout of one active plane using 14 extrusions versus one using 12 extrusions. Note that two extrusions bypass the center hole.

The extrusion width is determined by the width of the detector divided by the number of extrusions. The inner edge of the support shelf width is $4 \mathrm{~cm}$ from the steel octagon edge. Since the detector is symmetrical, the overall width of the active detector is $8 \mathrm{~cm}$ less than the absorber width. Dividing $792 \mathrm{~cm}$ by 14 extrusions results in each extrusion being $56.57 \mathrm{~cm}$ wide. The extrusion width will be reduced by manufacturing tolerances yet to be determined.

The number of cells in each extrusion is based on the number of channels in a photodetector and the number of planes per photo-detector. The cell width most closely approaching the nominal cell width of $2 \mathrm{~cm}$ is obtained when the 156 channel photodetector is divided between six module layers. Each module will have 26 cells. The result is a cell width of $2.17 \mathrm{~cm}$ including a $0.1 \mathrm{~cm}$ wall thickness. 
The number of cells change if 5 or 7 layers are connected to one photo-detector. If 5 layers are used per photo-detector, each module will have 31 cells. The cell width decreases to only $1.83 \mathrm{~cm}$ and the number of photo-detectors increases by $20 \%$. Increasing to 7 layers per photo-detector means each extrusion will have 22 cells but the cell size increases to $2.57 \mathrm{~cm}$ wide. This cell size is about $29 \%$ wider than nominal. The resolution of the last scenario may not be adequate for this experiment. More study is required to see what happens to the resolution with larger cell widths. Six planes per photo-detector is optimal for the nominal $2 \mathrm{~cm}$ cell width.

Some may have concerns that 14 extrusions per plane is not as good a number, electronically speaking, as the number 12 . However, the detector can be divided up in three sections of 360 planes each, which is divisible by 12 . Since each photo-detector is connected across six alternating planes, every 12 planes can be considered a mini-section, completely independent from the rest of the detector. Twenty-eight photo-detectors are required for 12 planes. A total of 840 photo-detectors are required in a section. This is evenly divisible by 12. In fact, the number of cells in every three planes is divisible by 12 . Therefore, the layout of 14 modules per plane should not be discounted for interfacing with digital equipment.

Determining the orientation of the extrusions to create a 2-D coordinate system depends mostly on the requirements for containment of the liquid scintillator. Placing the extrusion modules in a vertical position is optimal for containing the scintillator, as no top is required in this configuration. Since alternating planes must have different orientations, usually $90^{\circ}$ to the first, a symmetrical coordinate system is obtained by alternating active detector planes at $\pm 45^{\circ}$ from vertical. The planes alternate uniformly, creating an identical coordinate system for every pair of active detector planes. 


\subsection{CONTAINMENT OF THE LIQUID SCINTILLATOR}

This section describes the material best suited for containing the liquid scintillator based on a number of factors. The material must be inexpensive. The physical properties of the material must meet the reflectivity requirements in Chapter 2. This section also determines the mass and volume for the active detector plane.

The extrusion modules make up the containment vessel that holds the liquid scintillator for each active detector plane. The extrusions can be made of a metal such as aluminum or some type of plastic. An aluminum extrusion can have a very thin wall and still be structurally sound. A plastic extrusion requires that the walls be thicker than aluminum. Even so, with careful selection of the type of plastic, a plastic extrusion should be less expensive than aluminum.

The best plastic material is one that is inexpensive yet satisfies all the requirements for the experiment. The amount of plastic in a 10,000 metric ton detector may be over 225,000 kilograms. A savings of 45 cents per kilogram is a savings of over $\$ 100,000$ for the detector.

The plastic material must not be reactive with the liquid scintillator. Some absorption of the liquid scintillator in the plastic may occur. This will not be a problem unless actual leakage occurs or it significantly changes the size, shape, or strength of the plastic.

The reflectivity of the walls must be sufficiently high to gather enough photons to activate the photo-detectors-even if the light travels the entire length of the extrusion. The cell walls must have a reflectivity of greater than $95 \%$. Adding titanium-dioxide to the plastic will increase the reflectivity. 
One inexpensive material that is generally easy to extrude and retains it shape very well after the extrusion process is PVC. PVC can be bonded through a gluing process using adhesive made up of resins and solvents. PVC can also be bonded by melting or welding two pieces together. However, PVC give off chlorine gas when heated which is toxic.

PVC glue joints are most durable if the mating parts are cylindrical. Then the pressure applied from one surface to the mating surface is nearly constant at any point where the two surfaces meet. The geometry of the extrusions, previously proposed in Section 3.2, is much wider than its thickness. In this case, some external pressure must be applied for the gluing process to work well.

Polypropylene is an inexpensive plastic material with attractive structural attributes. Polypropylene is very pliable. It can stretch up to twice its length before breaking and it resists cracking. Although polypropylene cannot be glued like PVC, it adapts well to heat bonding techniques like welding. Polypropylene does not react with liquid scintillator made from a mineral oil base. More testing is required to see if is reacts with newer types of scintillators.

None of the inexpensive plastics is ideal for liquid scintillator containment. Polypropylene may be difficult to extrude in the optimal width and cell size. High density polyethylene may be easier to extrude for the optimal shape but it may absorb the liquid scintillator more readily than polypropylene. For this reason, polypropylene is considered a better material despite the challenges of extruding it.

Plastics, by definition, tend to flow or creep. By containing the modules between the planes of steel, the amount of creep can be limited. Limiting the free space between the steel planes is one way to limit the expansion of the extrusions due to creep. 
Steel plates from the steel mill are not perfectly flat. The steel is manufactured to a waviness tolerance. This tolerance is used to design the required free space between the steel planes and the extrusions. If the extrusion modules are allowed to be locally compressed $1 \mathrm{~mm}$ or $2 \mathrm{~mm}$ by the waviness of the steel plane, the designed free space can be reduced to a minimum.

The governing factor for the plastic extrusion wall thickness is not the structural strength of the extrusion but the wall thickness needed during the critical extrusion phase. Plastics have a temperature range in which they can be molded, shaped, or worked in some manner. As a plastic is extruded, it becomes workable and has little internal strength to maintain its shape. The shape must be mechanically maintained using a vacuum system until the plastic material cools sufficiently to keep its shape on its own.

Each extrusion has both interior and exterior walls. The exterior wall thickness is chosen to be $1.5 \mathrm{~mm}$. The internal walls that divide the extrusion into several cells are chosen to be about $1 \mathrm{~mm}$ thick. Figure 10 shows a $2 \mathrm{~cm}$ thick extrusion that has 26 cells and a width of $56.57 \mathrm{~cm}$. The cross-sectional area of the extrusion is $21.731 \mathrm{~cm}^{2}$. The material volume of an 8 meter extrusion is $0.0174 \mathrm{~m}^{3}$. For polypropylene with a density 900 kilograms per cubic meter, the mass of an 8 meter extrusion is 15.65 kilograms $(34.42$ pounds). An 8 meter extrusion is manageable by two people if it is carried on edge.

Each plane contains 14 extrusions between 4.3 meters and 8 meters long. These dimensions do not include the top manifold. The top of each extrusion is cut parallel to the octagon edge similar to the bottom cuts described in Section 3.2. The cuts are made so that the extrusion end is slightly inside the octagon area. The resulting area of the extrusion plane nearly matches the area of the octagon. The mass of the entire plane 
without liquid scintillator is 180 kilograms (400 pounds). Refer to Section 9.2.2, Extrusion Area, Volume, and Mass in Appendix B for mass calculations.

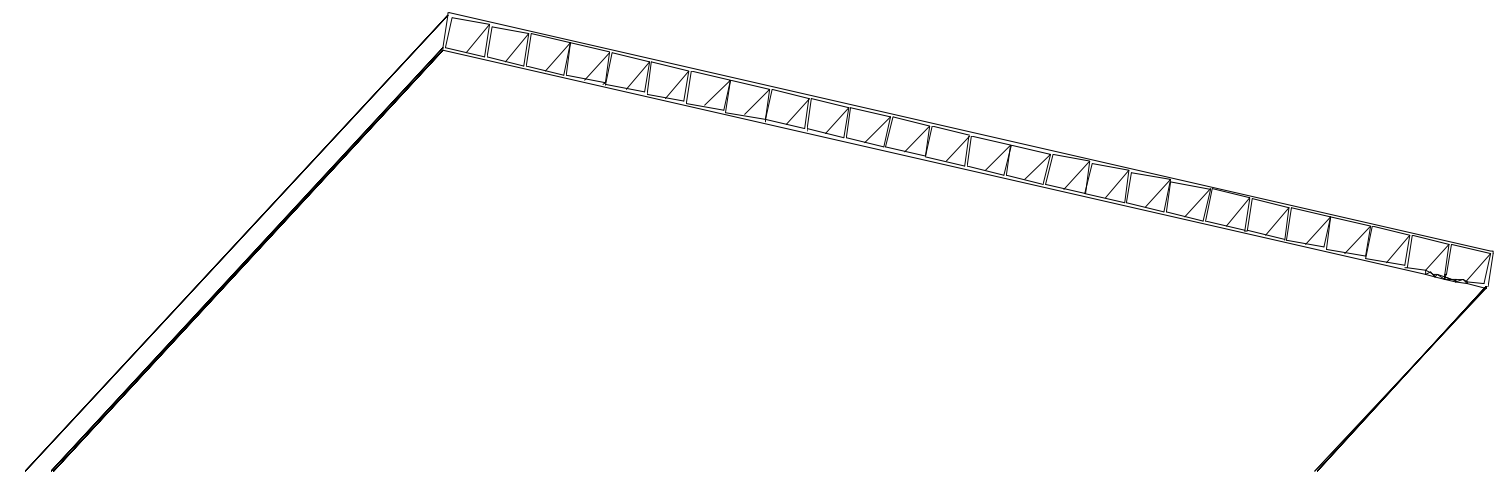

Fig 10. Cross section of one extrusion module with 26 cells.

The liquid scintillator nominally fills the channels within each extrusion. The density of the scintillator is about the same as the polypropylene. The mass of both the scintillator and the plastic extrusions can be calculated together using the area of the octagon, the thickness of the plane, and the density of both materials. The mass of each active detector plane is about $950 \mathrm{Kg}(2100 \mathrm{lbm})$. The mass of the scintillator alone is $770 \mathrm{~kg}$ or 1700 lbm. Eighty hundred forty liters (221.9 gallons) of liquid scintillator are required to fill each plane. About 907,500 liters (240,000 gallons) are required to fill the entire detector.

\subsection{BYPASS DESIGN}

The design of a magnetized detector requires a hole through the center of the detector for an electrical coil. Some of the cells in the extrusion modules adjoining the hole are cut in two by the hole. In an earlier design, the affected cells required fibers to exit from the bottom of the extrusions before attaching to a photo-detector. This created a potential for leakage that should be avoided if possible. In addition, the extra photo-detectors added cost to the detector. 
One way to eliminate the fibers from the bottom and the associated photo-detectors is to route the fibers around the central hole. Two of the 14 extrusions in each plane require this modification. This section details the modification process.

A bypass with an integral groove is designed for routing the fibers around the center hole. The fibers from the affected cells enter the groove. The fibers are routed around the central hole via the groove. On the lower side of the hole, the fibers exit the groove to their respective cells. The fibers continue their journey to the bottom of the cells.

Figure 11 shows the bypass positioned in the extrusion module with the fibers installed in the bypass groove. The interior of the extrusion is exposed to reveal how the fibers are routed from their cells into the groove and back out to their corresponding cells. The phantom circle indicates the position of the center hole in the detector.

Figure 12 shows a close-up of the bottom area of the bypass. The transition of the fibers from the bypass groove to the cells is clarified in this close-up.

To prepare for the bypass, an arc with a radius slightly larger than the center hole is cut in the extrusion. A jig is placed over the extrusion as a template for the proper contour. A blade follows the template to cut out the section of the extrusion required for the bypass.

The bypass is slightly larger than the section cut from the extrusion. This allows the extrusion to overlap the bypass and cover the groove. The internal cell walls are cut back about $2 \mathrm{~cm}$ from the edge of the arc cut to allow for installation of the bypass. The bypass can then be welded to the overlapping part of the extrusion. The bypass installation procedure is discussed in more detail in Section 4.3. 


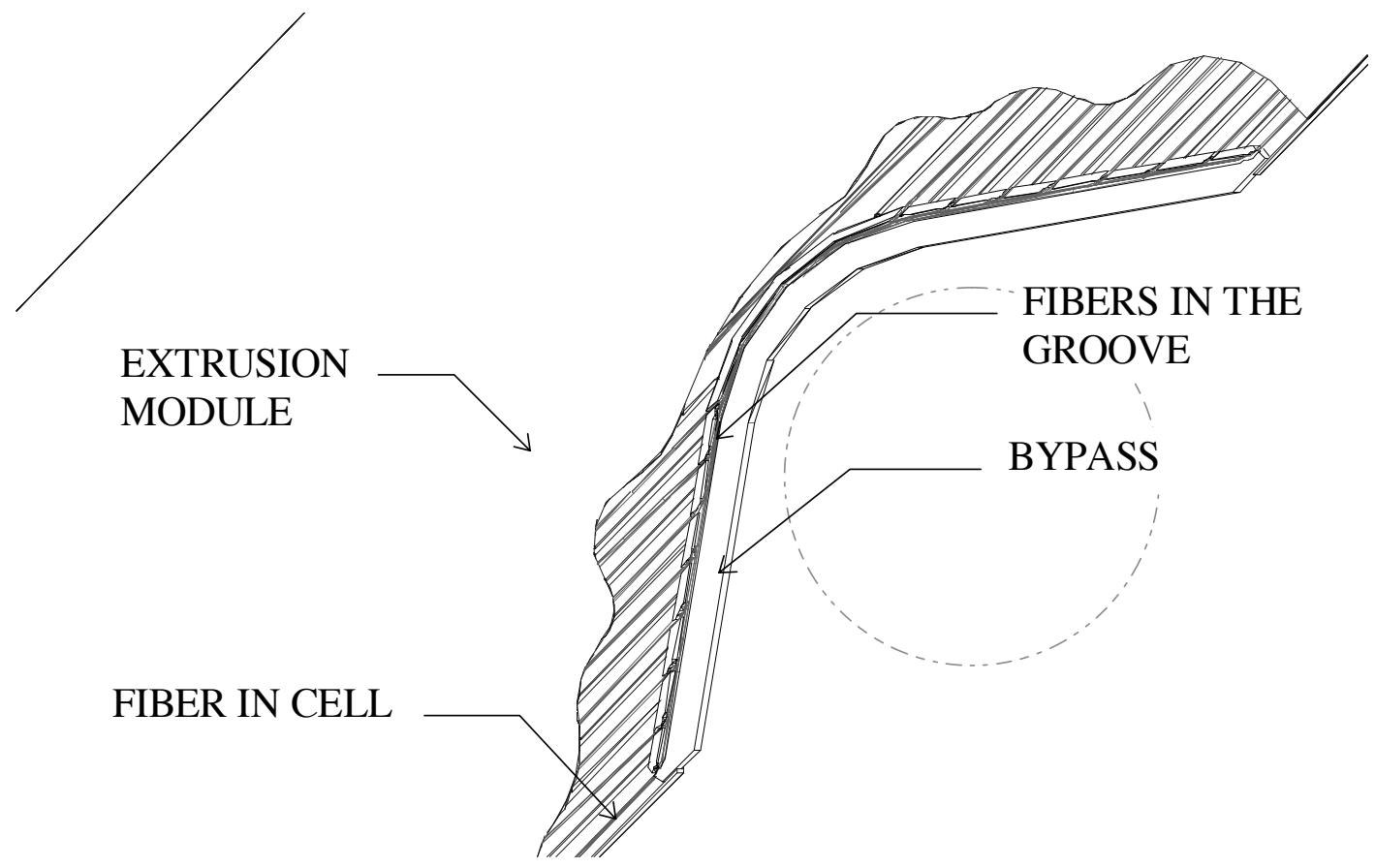

Figure 11. View of how the bypass fits into the extrusion and routes the fibers around the center hole.

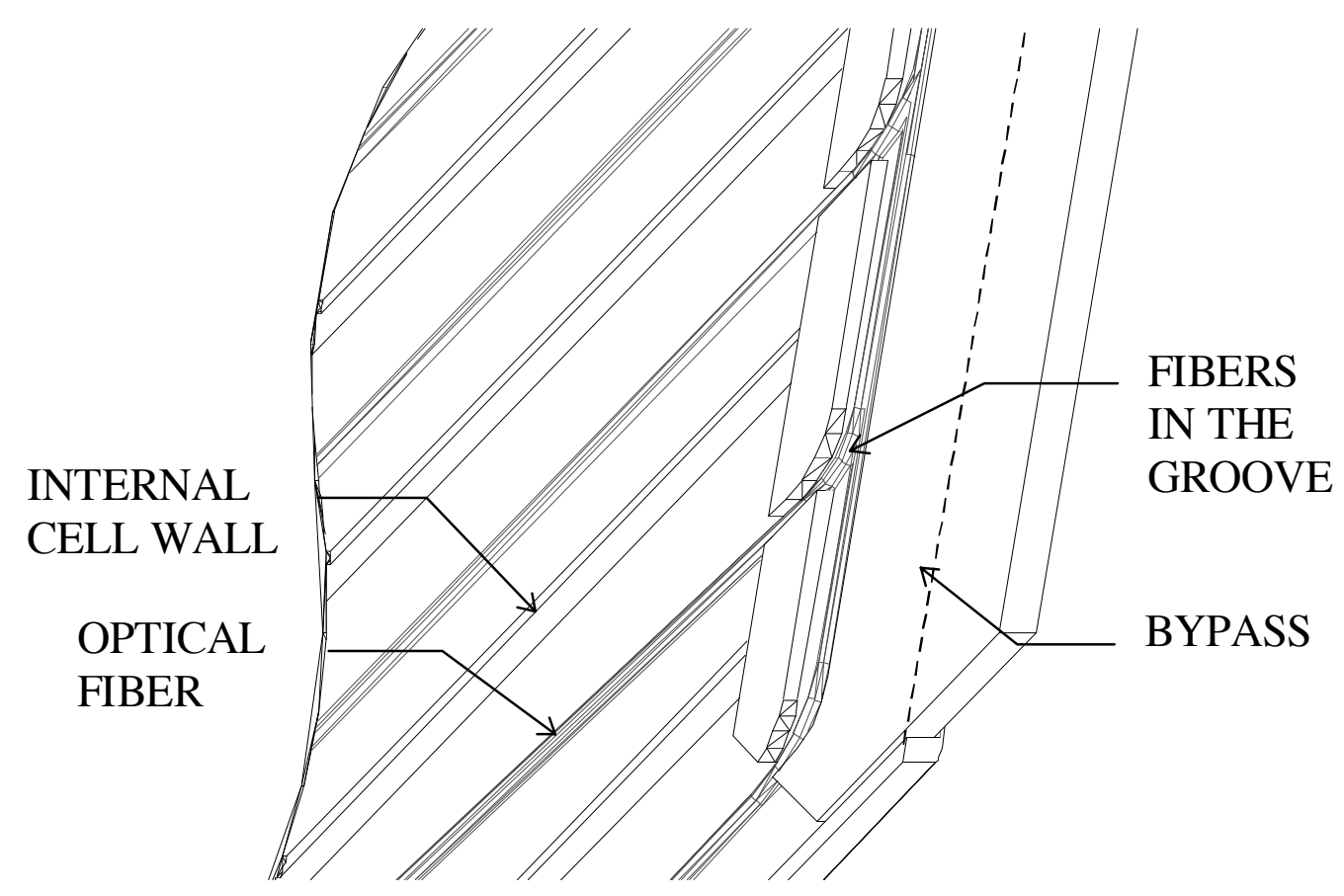

Figure 12. Close-up view of one area of the bypass showing how the fibers transition from the bypass groove to a cell. 


\section{MANUFACTURING CONSIDERATIONS}

This chapter discusses several issues concerning the manufacture of extrusion modules. The extrusion modules are defined as an assembled and completely sealed unit containing an extrusion, an endcap, a manifold, and optical fibers. The modules are assembled in a factory or manufacturing facility. The factory may be a building off-site or at the detector location if enough room is available.

This chapter describes the assembly process of the extrusion modules. Section 4.1 details the bottom endcap seal of the extrusion. Section 4.2 describes the fiber insertion process into the top of the extrusion and the manifold. The manifold is welded to the top of the extrusion. Section 4.3 discusses the installation of the fiber bypass which is required in some extrusions. Finally, Section 4.4 details the final preparations for the modules by machining the fibers flush with the top of manifold.

\subsection{SEALING THE BOTTOM OF THE EXTRUSIONS}

This section discusses how the bottom of the extrusion modules are sealed. The design of the endcap and the mold are discussed in Section 4.1.1. Section 4.1.2 explains the melting of endcap. The attachment of the endcap to the extrusion is discussed in Section 4.1.3. Alternate methods of sealing the bottom are presented in Section 4.1.4.

\subsubsection{ENDCAP AND MOLD DESIGN}

The endcaps used to seal the bottom of the extrusions were designed for maximum simplicity. The endcaps are made from the same material as the extrusions. They are designed to be made from stock sheet material to reduce machining costs and eliminate the 
cost of an injection mold. The profile of the endcap is slightly smaller than the crosssectional area of each extrusion. The thickness of the endcaps is $9.5 \mathrm{~mm}(3 / 8$ "). The only requirement is that the thickness be sufficient to make a good bond with the extrusion.

Two different profiles are used for endcaps. The first profile width is slightly shorter than the width of the extrusion. The endcap expands enough during heating to cover the end of the extrusion. This endcap is attached to the extrusions that are cut perpendicular to their width. The other endcap is cut longer to fit the extrusions cut at a $45^{\circ}$ angle.

The bottom of each extrusion is completely sealed using a thermal process. An endcap is placed in a mold which in turn is placed in an oven at a temperature higher than the melting temperature of the endcap. After a few minutes, the endcap melts. The mold containing the endcap is removed from the oven and attached to the end of the extrusion. The molten endcap contains enough heat to melt the mating surfaces of the extrusion. This creates a permanent bond nearly as strong as the original material.

Figure 13 shows the mold and endcap. The mold is made from a 2" x 1" aluminum bar stock. The length of the mold depends on the length of the endcap. A groove is milled down the center of the mold that is slightly wider and deeper than the endcap. The mold is designed to contain sufficient mass to retain the heat of the molten endcap long enough to create an integral bond between the endcap and the extrusion.

A layer of aluminum foil between the mold and the endcap acts as a release medium. The foil sticks to the endcap but not to the mold. Alternatively, the mold may have a permanent Teflon coating but aluminum foil releases the endcap from the mold easier. 


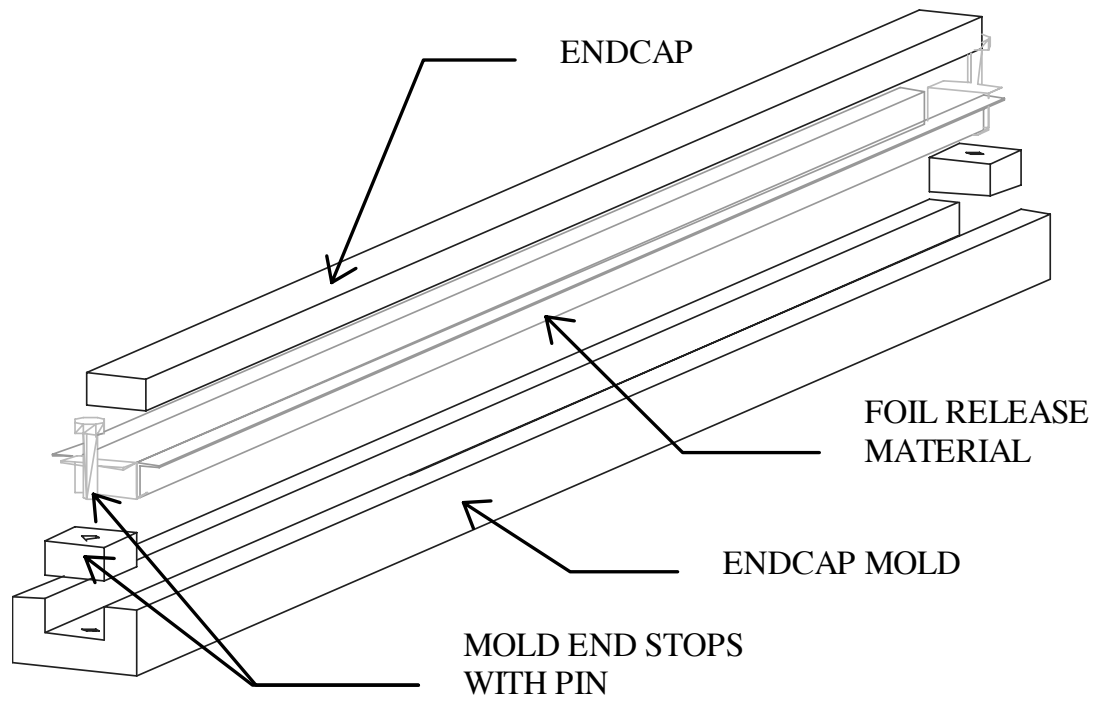

Figure 13. The endcap mold and endcap with the aluminum foil release material.

The application of aluminum foil in the mold is important to achieve a good release for the endcap from the mold. The foil is cut to the exact length of the channel and laid inside the channel with the edges folded over the outside of the mold. The plastic endcap is centered in the mold channel ${ }^{1}$. Small pieces of foil are then placed over the mold end stops to complete the release surface.

\subsubsection{PREPARING THE MOLTEN ENDCAP}

The endcap must be prepared correctly to ensure a good bond to the extrusion. The endcap is placed in an oven that has evenly distributed heat. Experimentation revealed that monitoring the texture of the endcap surface, the temperature, and the time the endcap is in the oven are important.

The oven used in the bonding tests was a standard kitchen oven with a convection fan for even heating. The oven specifically used for the experiment is a Kitchen-Aid Superba 27

\footnotetext{
${ }^{1}$ It may be easier to wrap the endcap with the foil before carefully placing it in the mold to reduce the chances of burning the fingers.
} 
thermal-convection oven. The best results were obtained using the Convection Roast setting at $245 \mathrm{C}\left(475^{\circ} \mathrm{F}\right)$.

The time required to heat the endcap to the desired temperature depends on the starting temperature of the mold. The first run of the day, starting with a cold mold, required 60 minutes to melt the endcap. The second run only required 30 minutes because the mold retained its heat from the first run.

The surface of the endcap was monitored about 25 minutes into the heating process. The endcap is ready to be applied when the surface appears somewhat shinier than its original state. The top surface of the endcap can be tested with the tip of an awl or similar metal object. If the plastic adheres to the awl, the endcap is ready. If the endcap is left in the oven too long, polypropylene begins to separate and a oily-yellowish film appears on the surface. The endcap will no longer make an good bond to the extrusion if the film appears.

It is important to note that the oily-yellowish film appears as a function of time and temperature. The film may appear if the temperature of polypropylene is only $400^{\circ} \mathrm{F}$ and that temperature is maintained for a long time. However, polypropylene can be heated to $500^{\circ} \mathrm{F}$ before the film appears if it reaches that temperature quickly. A minimum temperature of $450^{\circ} \mathrm{F}$ is required for melting the mating surfaces of the extrusion.

The temperature of the oven determines the rate at which the endcap heats up. If the oven is set too low, the film will start to appear on the surface before the endcap contains enough thermal energy to melt the extrusion mating surfaces. If the oven is set too high, the endcap will retain too much heat. In this case, the walls of the extrusion become thin and weak near the area where the endcap bonds to the extrusion walls. 


\subsubsection{APPLYING THE ENDCAP TO THE EXTRUSION}

The modules may be up to 8 meters long and unwieldy. A practical method for attaching the endcap is to lay the extrusion on a horizontal surface like a table. The end of the extrusion must be cut in a jig to ensure a straight clean surface to mate with the endcap. The mold containing the molten endcap is also affixed to a jig to ensure proper alignment of the endcap with the extrusion. The fixture, shown in Fig. 14, also provides the proper force to create a good bond between the endcap and the extrusion. The detailed attachment process is described below.

The extrusion is placed on the table and cut to length. A straight edge jig is placed over the extrusion and clamped in place. A utility knife is drawn across the extrusion using the straight edge jig as a guide. Only a few passes are required to cut through the extrusion without much force.

The extrusion is placed in the jig shown in Fig. 14. Two alignment stops provide a guide for placing the extrusion in the proper position for receiving the molten endcap. The extrusion is clamped to the table in position and the stops are rotated down out of the way. Once the extrusion is clamped in place, a tool is used to cut out the cell dividers to a depth of $2 \mathrm{~cm}$. The cell dividers are cut out to allow the liquid scintillator to flow freely from cell to cell at the bottom of the extrusion. This is most important during the filling stage to prevent trapping of air in the extrusions.

Once the divider walls are cut, the jig fixture in Fig. 14 is used for attaching the endcap. The mold is taken from the oven and placed in the fixture ${ }^{2}$. The mold is oriented so the molten endcap is facing up. The handle on the fixture now rotates the mold around a $90^{\circ}$

\footnotetext{
${ }^{2}$ An alternative to heating the mold in the oven is to design an integral heater built into the mold.
} 
arc until the molten endcap comes in contact with the extrusion. The heat from the endcap is enough to melt the end of the extrusion creating a permanent bond. The viscosity of the molten plastic is similar to very cold molasses, so holding the mold vertically causes little sagging in the endcap.

A mass of $4 \mathrm{~kg}$ on the end of each handle provides the proper force to attach the endcap to the extrusion. The length of the lever weight arms are 1.5 times longer than the arm of the mold. The force applied to the endcap is approximately $120 \mathrm{~N}(27 \mathrm{lbf})$. After 50 seconds, the handle is placed in the upright position and the mold returns to its home position. The endcap releases from the mold and stays attached to the extrusion.

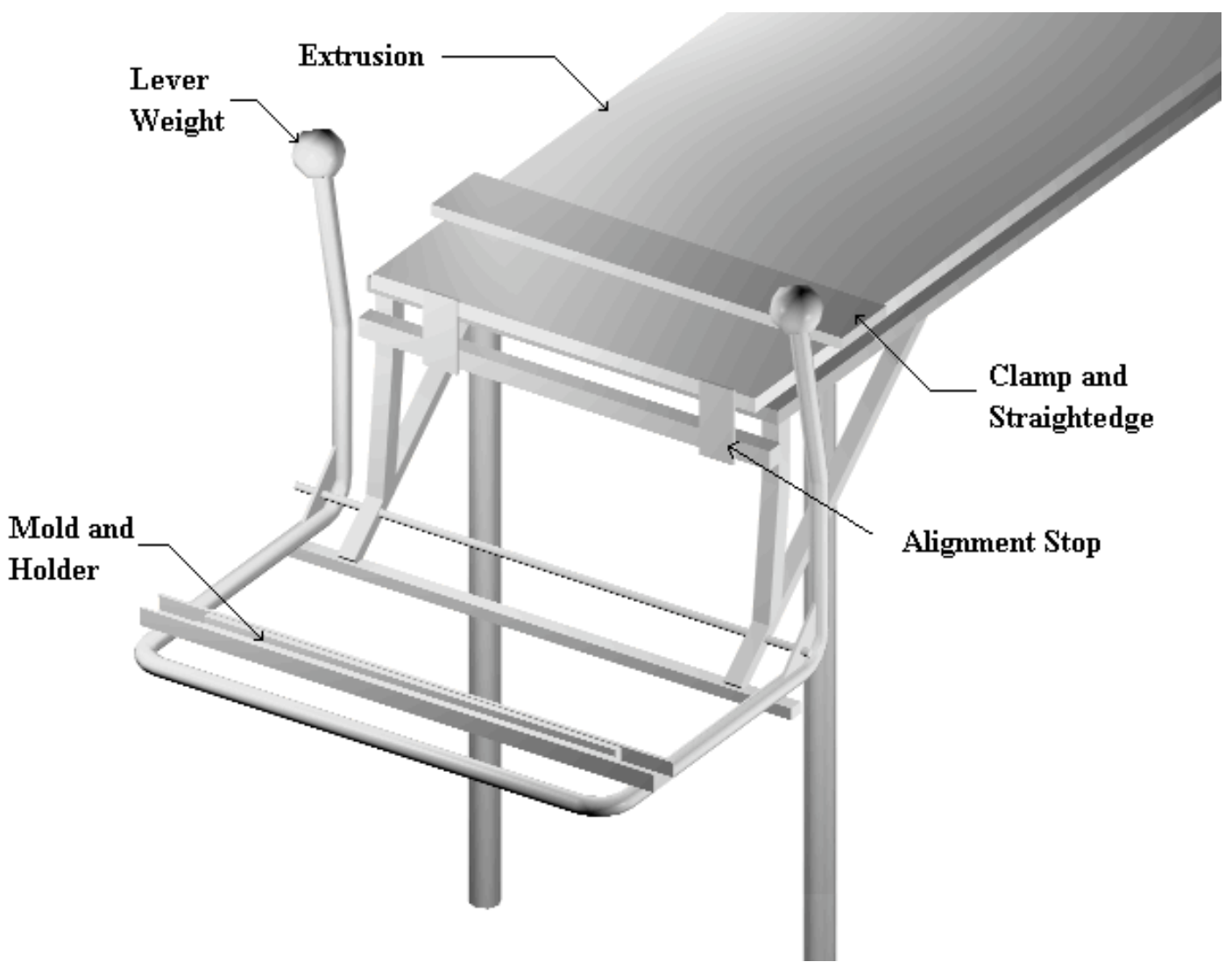

Figure 14. View of endcap applier jig and the extrusion on a table. 
The mold retains its heat for the next endcap. The temperature of the mold is now slightly lower than the melting temperature of the endcap. Another layer of foil is placed in the mold along with another endcap and the process is repeated.

After molding, the endcap is trimmed to the same width as the extrusion. The modules must fit tightly beside each other or the dimensional build up at the endcaps will force the last modules to be outside the octagon. The endcaps can be thicker than the extrusion modules because of the extra airspace that is designed between the steel plates and the modules.

The process for attaching endcaps is the same for both the perpendicular cut and the $45^{\circ}$ cut extrusions. The two types need different fixturing to accommodate the different endcap lengths and different geometry. The $45^{\circ}$ extrusion is less forgiving of the attachment process because of the angle. The normal force of attaching the endcap may push the melted mating surfaces of the extrusion beyond the acute angle of the extrusion. Care must be taken to keep the attachment fixture in proper alignment.

The endcap must be attached to the extrusion as the first step. If the endcap is attached after the opposite end is sealed, the internal pressure will increase from the heat of the endcap. The increase in air pressure could cause the molten walls to blow out during the bonding process.

\subsubsection{ALTERNATE ENDCAP SEAL DESIGNS}

This section provides two alternate seal design for the bottom end of the extrusions. The first design is an endcap similar to the molten endcap design. This endcap is slightly smaller in size to fit inside the extrusion after the cell walls have been cut. The endcap is welded in place using polypropylene welding rod. The second design is a flexible seal that 
attaches to the bottom of the extrusion. This design is used fibers exit the bottom of the modules.

One alternate method of sealing the bottom of the extrusion is to weld on an endcap. The endcap width and length are sized just small enough to fit inside the extrusion. The endcap thickness is $9.5 \mathrm{~mm}$ as in the molten endcap design. The cell divider walls are cut flush with the inside of the exterior walls to a depth of $2 \mathrm{~cm}$ to enable the endcap to fit inside. The endcap is positioned inside the extrusion, leaving half of the endcap protruding from the bottom. Then the endcap is welded with a fillet weld using a hot air welder and plastic welding rod. The extra space between the endcap and the internal divider walls allow for liquid scintillator to flow freely from cell to cell during filling.

In an earlier design, the extrusions had fibers coming through both the top and bottom of the extrusions. The above method of thermo-forming could not be used. Some other methods of sealing the fibers were explored.

Another thermo-forming method was proposed to allow fibers to exit the bottom end of the module. In this case, the end of the extrusion would be cut square or at $45^{\circ}$ as before. The dividers would also cut back. Instead of attaching an endcap, the end of the extrusion would be inserted in a heated mold. The end of the extrusion would be melted and shaped to hold a molded seal . The seal would be made from Viton, which is a flexible material known to resist the effects of mineral based liquid scintillator. The seal would be a single piece unit that fits into the remolded end of the extrusion module. The seal would be designed to fit tightly against the module. The fibers exit through holes in cone shaped protrusions facing towards the inside of the module. The hydrostatic pressure from the liquid scintillator exerts pressure on the surface of the cone shapes causing the seal to squeeze against the fiber. 
The single piece cone shaped seal design in Fig. 15 was never built. If a scintillator detector is built that includes photo-detectors on both ends of the extrusion modules, this design should be explored.

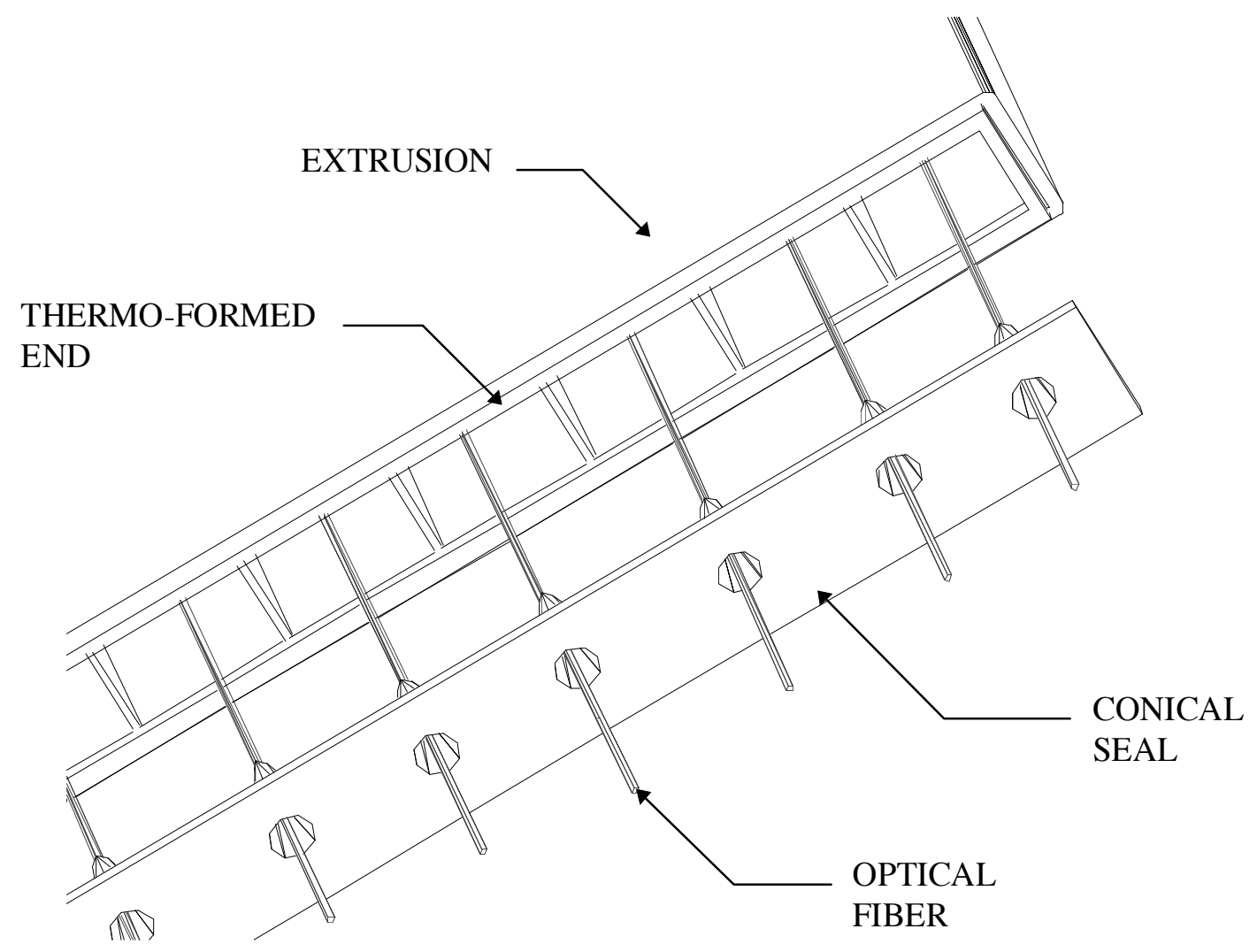

Figure 15. Conical seal on the bottom of the module to seal around fibers.

\subsection{THE TOP OF THE EXTRUSION MODULE}

This section describes the assembly of the top of the extrusion module. First, the fibers are inserted into the extrusion using a fiber inserter. Then, the fibers are inserted into the manifold. The manifold is welded to the top of the extrusion. 
The detector requires that every extrusion have an optical fiber running the entire length of each cell. The end of each fiber at the bottom of each cell will have one end polished and mirrored. The mirroring reflects light in the fiber which increases light yields to the photodetector $^{3}$. The coating is applied using an aluminum application process inside a vacuum. After the mirroring process, the end of the fiber is sealed with epoxy.

The fibers are inserted inside each cell mirror end first. Approximately 400,000 fibers are installed in a detector of this scale. Some fibers must be inserted 8 meters. A mechanized method of inserting fiber is important to reduce the assembly time. The motorized fiber inserter in Fig. 16 will insert all the fibers in one extrusion at the same time at a rate of about 1 foot per second. ${ }^{4}$

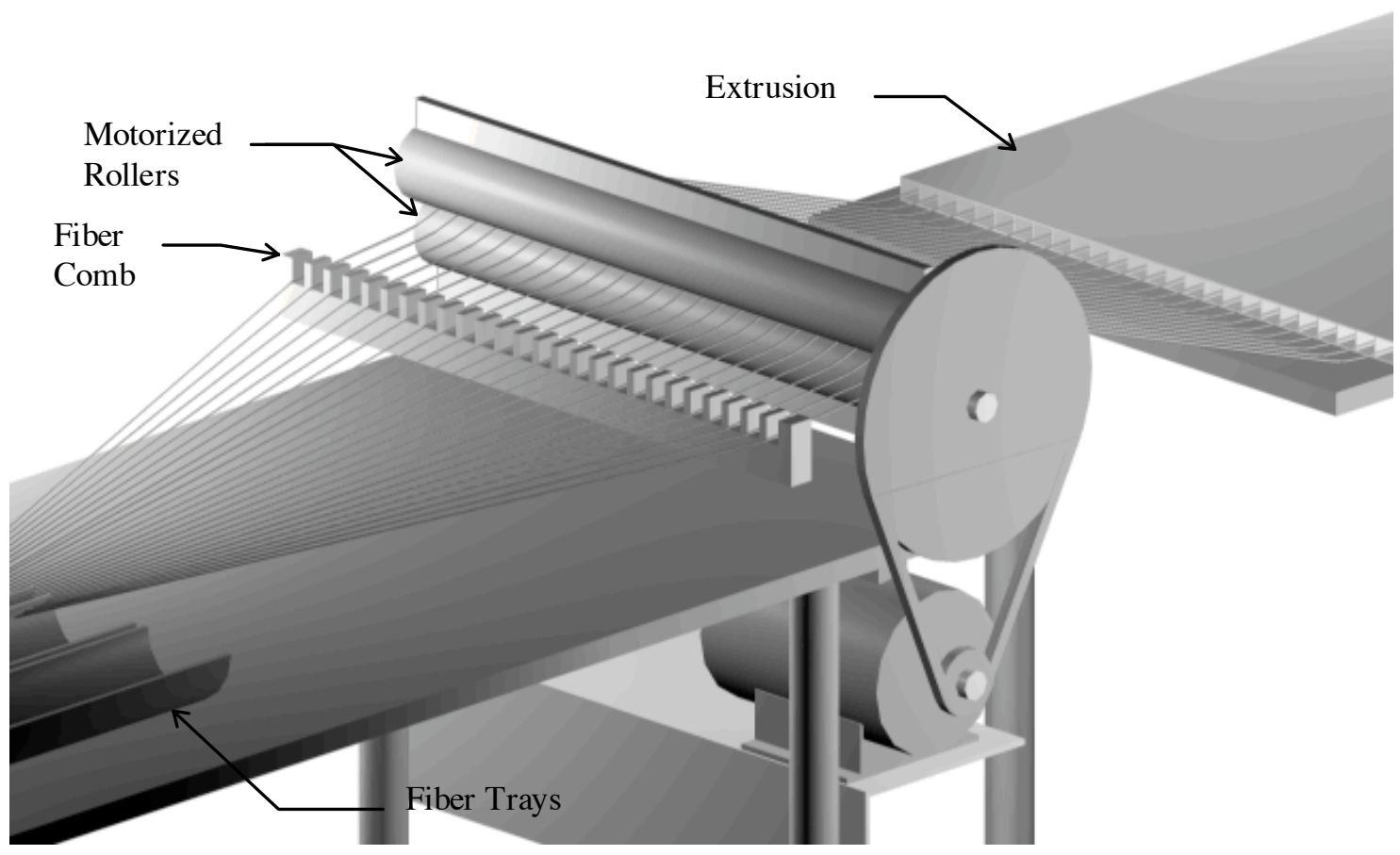

Figure 16. Motorized fiber inserter pushing fibers into an extrusion module.

\footnotetext{
${ }^{3}$ Increased light yield is defined as more light photons emanating from the optical fibers to the photo-detector.

${ }^{4}$ Modules with $45^{\circ}$ ends will require more care to ensure all the fibers are pushed to the bottom of each cell.
} 
In the factory, four different lengths of fibers are utilized, one for each extrusion length. All fibers of the same lengths are placed in a long tray on top of a long table which can be rolled around. A tray can be made from PVC pipe cut in half lengthwise. Four different trays on the table hold all the fiber lengths needed for any extrusion. The fiber inserter is also attached to this table. The table can then be placed end to end with the extrusion that requires the fibers.

The fibers are first inserted a few inches into each cell of the extrusion by hand. The top roller of the fiber inserter is removed or tipped up out of the way. The fibers are placed through a fiber comb fixture to keep the fibers from being tangled during the insertion process. The top roller is then placed over all the fibers. The top roller and a lower roller provide the motion for inserting the fibers similar to the old style washing machines with hand wringers.

A means of shutting off the fiber inserter must still be developed when the fibers reach the end of their travel. When the fibers reach the end, they could bow up and activate a shutoff for the inserter. The shutoff could be in the form of an electric eye beam. Another method could count the number of turns on the inserter. This method requires four different settings for the different fiber lengths.

Once the fibers have been fully inserted in each extrusion cell, each fiber is marked with a magic marker showing the point on the fiber that matches with the end of the extrusion. The mark is used to gauge how far the fiber is to be inserted in the manifold that covers the top of the extrusion.

Figure 17 shows how the fibers are placed in the manifold. The fibers must be manually threaded through the manifold into holes that correspond to each extrusion cell. The complexity of the insertion operation makes it difficult to automate this operation. One 
side of the manifold must be kept open during this process to allow access to each fiber. The fibers are threaded one by one through the lower holes in the manifold. Then the fibers are threaded through the corresponding upper hole using the access area on the top side.

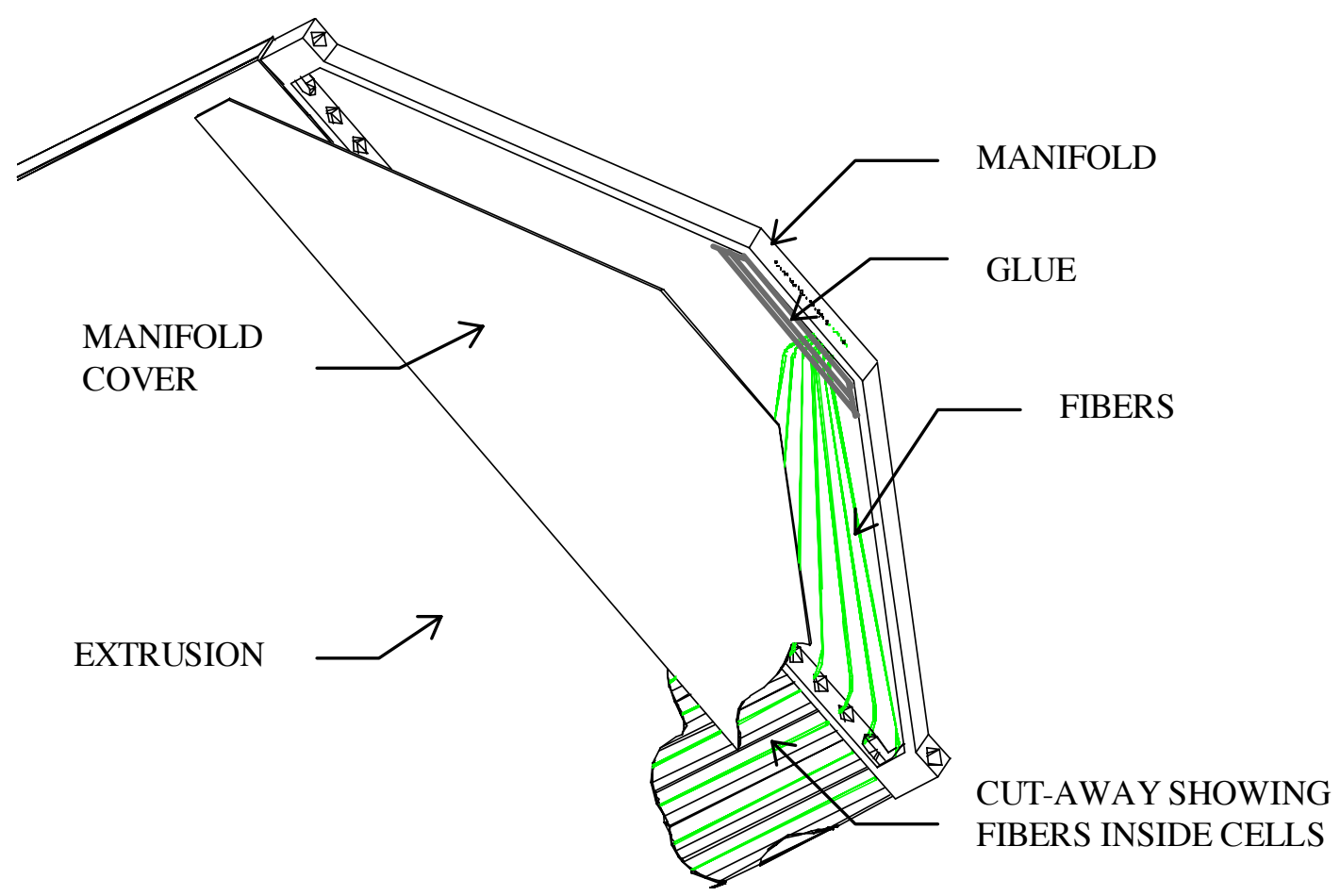

Figure 17. Manifold attached to the end of the extrusion showing the fibers and cover.

After the fibers have been threaded in their proper holes, the fibers are glued in place. The manifold with the fibers through the holes is placed in a fixture with the fiber ends pointing downward. Glue is poured into the manifold. The glue fills the volume where the fibers exit through the manifold. The glue keeps the fibers from moving and also seals the holes to prevent scintillator leakage. At the top of each module, the liquid scintillator has no hydrostatic pressure so the glue mainly serves to keep the fibers in place. Once the fibers are glued in place, a plastic cover seals the open side of the manifold. 
The manifold and its cover are attached to the extrusion by hot air welding using plastic welding rod. Another method of attachment under investigation is 'hot gluing' - using molten plastic as a glue similar to the hot glue guns currently on the market. In both methods, molten plastic causes a small area of contact on the manifold, cover, and the extrusion to fuse together by melting with the molten 'glue'.

\subsection{INSTALLING THE BYPASS}

The bypass is installed in the extrusion in conjunction with the fibers and manifold. Only 2 of the 14 extrusion modules per plane require the bypass. The bypass installation and installation of the fibers must be coordinated to ensure that the fibers are in their proper position of each cell.

Before the fibers are inserted in the center extrusion modules, the extrusions must be prepared for the bypass. An arc is cut out of each extrusion module as described in Section 3.4. This exposes the inside of each extrusion.

After the cut-out is prepared and the internal cell walls are cut back about $2 \mathrm{~cm}$, the fibers are inserted using the method described in Section 4.2. Care must be exercised when the fibers exit the bypass area to prevent broken fibers. The fibers that exit the bypass area must be inserted in their corresponding cells for the rest of the insertion process.

The exposed fibers are manually conformed to the arc shape cut in the extrusion before the top position of the fibers are marked. This ensures that the fibers will reach the bottom of all cells when the final assembly is complete. The fibers are then inserted in the manifold and the manifold is welded into place before the bypass is installed.

When the manifold is installed, the exposed fibers will tend to arc in the bypass area because they are too long to lie straight. The bypass is placed below the horizontal 
extrusion and the fibers are fitted into the bypass groove as shown in Fig. 18. Then, the bypass and fibers are manipulated, as a whole, into the opening of the extrusion. The bypass is welded to the extrusion around the perimeter to make a liquid tight seal.

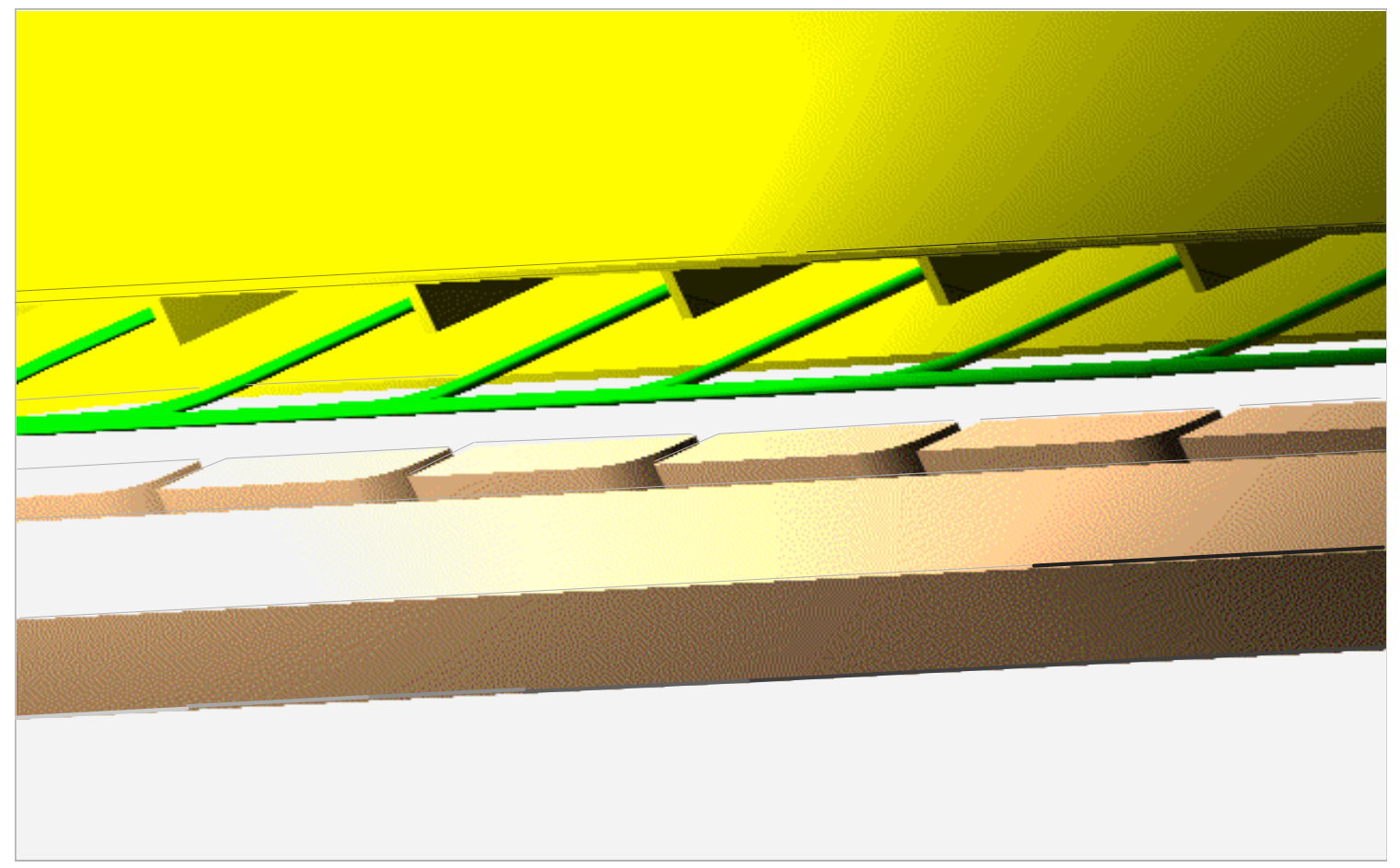

Figure 18. The bypass is shown below the extrusion module and fibers in preparation for the fibers to be inserted into the bypass. The bypass is then inserted into the extrusion and welded around the perimeter.

\subsection{FINAL PREPARATION AND QUALITY ASSURANCE TESTING}

This section describes the final machining on the modules required to optimize the light transfer from the fibers in the manifold to the light guide. In addition, two quality assurance tests are performed to certify the modules are ready for installation. The first test ensures the quality of the fibers installed in the modules. The second test is a pressure leak test to certify that the modules will not leak when filled with liquid scintillator. 
When the extrusion modules are complete and the glue holding the fibers has cured, the modules undergo final preparation before shipping. The end of the manifold where the fibers terminate is fly cut on a mill to a smooth surface and polished. The polish is necessary to provide good transfer of light to the light guide harness and consequently to the photo-detector. If the surface is not polished, light will be lost and the detector will not be sensitive enough to observe neutrino interactions.

Alternatively, the fibers and manifold may be flycut before it is welded to the extrusion. Then the manifold can be clamped in a vise that is in turn mounted in the mill. However, extreme care must be taken to ensure none of the exposed fibers are damaged.

The modules are tested to make sure they meet the specifications for the experiment. Each module is pressure tested to ensure it is leak-free before it is installed in the detector. The module is pressurized to 40 psi. A liquid leak detector solution is applied to all the welded and thermo-formed seams. Any bubbling of the solution indicates a leak that must be repaired before mounting the module to the absorber.

In addition to the pressure test, a light test is performed to check the integrity of all the fibers in the module. A device that contains two small fibers is placed over the end of each fiber in a module. One fiber in the device is used as a light source to flash photons into the fibers. The photons bounce off the mirrored surface at the other end of the fiber and return to be picked by the second fiber in the device. The device measures the light from the module fiber to determine its integrity. If the test fails, the module must be repaired or in some cases-replaced. 


\section{ASSEMBLY OF THE DETECTOR}

This chapter discusses the movements of the extrusion modules after the manufacturing process is complete. If the factory is off site, the modules will require transportation to the site. Section 5.1 describes how the modules are transported to the site for storage in containers until needed for installation. Scintillator will also be transported to the site for storage. Section 5.2 discusses how the modules are integrated with the steel planes and assembled in the detector.

\subsection{TRANSPORTING TO THE DETECTOR SITE AND STORAGE}

Once the extrusion modules pass the quality assurance tests described in Section 4.4, they are prepared for shipping to the detector site for assembly. The modules are placed in a containers for shipping. Each container is approximately $0.6 \mathrm{~m}$ wide by $0.6 \mathrm{~m}$ high and $8.5 \mathrm{~m}$ long. Each container is able to hold enough modules for 2 planes. The containers are designed to allow lifting by a hoist.

The containers of completed modules are shipped from the factory to the detector site. The containers are loaded on a truck for the journey to the site. When the truck gets to the site, the containers are hoisted from the truck and taken directly underground for storage.

Shortage of storage space at the detector site will require frequent trips from the factory. Only a few days supply will be able to be stored at the site. Two weeks supply at two planes per day requires a space of $1.2 \mathrm{~m}$ wide, $3 \mathrm{~m}$ high, and $8.5 \mathrm{~m}$ long (4'x 10'x 28').

Liquid scintillator must also be stored at the site in limited supply. Each plane requires $840 \mathrm{~L}$ (221 gal.) of scintillator (4 -55 gal. barrels). Forty barrels of scintillator is required 
for one weeks supply. The storage space for the scintillator is $1.2 \mathrm{~m}$ wide, $2.8 \mathrm{~m}$ high, and $6.1 \mathrm{~m}$ long (4’x 6’x 20’).

Storage space for the steel plates is next to the assembly area. The steel plates are brought in from the mine shaft on a monorail system. The steel plates are stored horizontally on the floor or on edge. Eight steel plates are required for each steel plane. A two week supply consists of 160 plates. The steel required for two weeks is $2 \mathrm{~m} \mathrm{x} 18 \mathrm{~m} \mathrm{x} 0.8 \mathrm{~m}$ ( $6.5^{\prime} \mathrm{x}$ $\left.60^{\prime} \mathrm{x} 2.63^{\prime}\right)$.

Figure 19 shows an illustration of how the assembly area of the underground cavern might appear including storage and 3 assembly stations. The steel planes are assembled on strongbacks ${ }^{5}$ which move on rails in the floor. The monorail system used to transport the steel into the cavern is not shown.

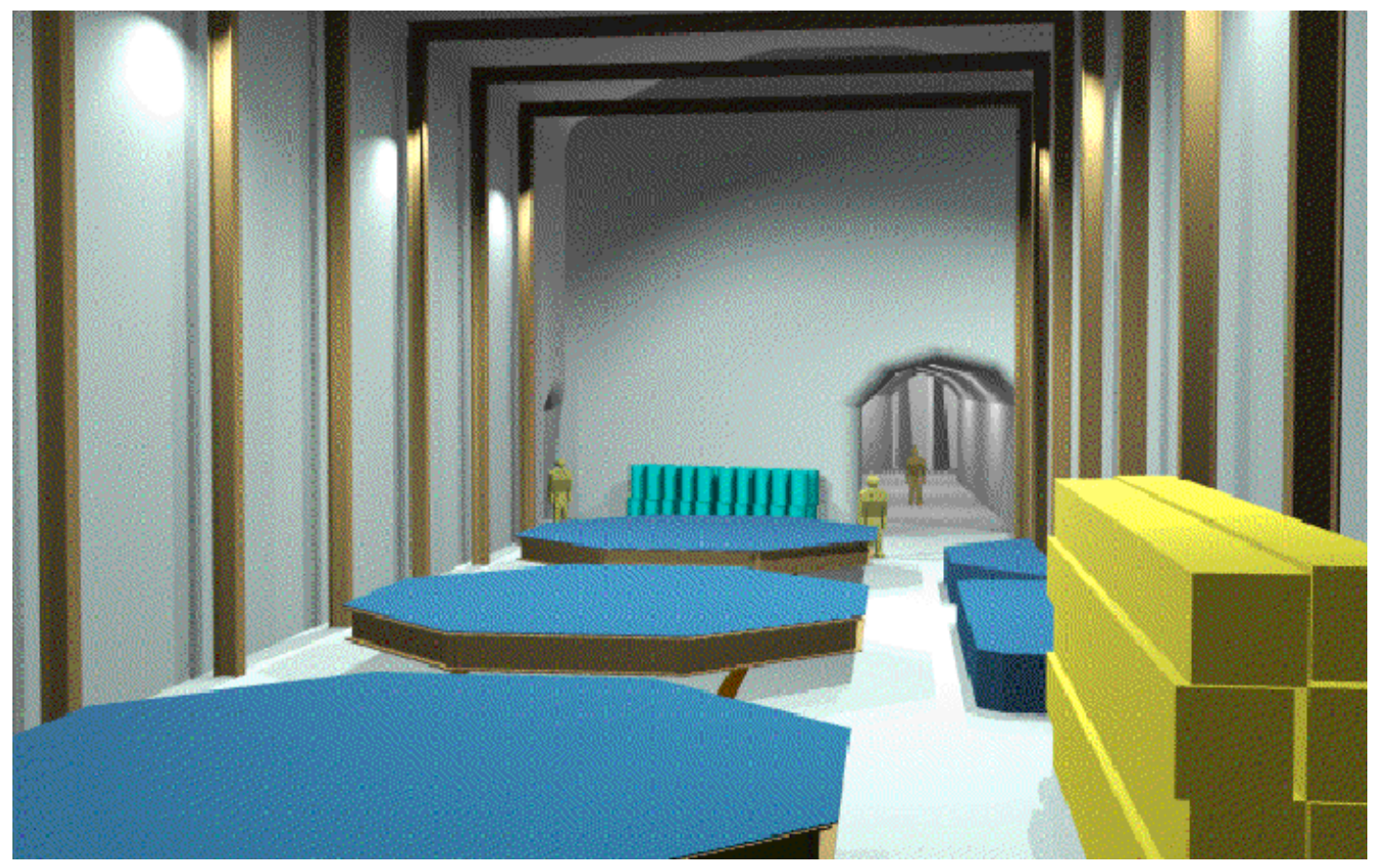

Figure 19. Floorplan of mine with 3 assembly stations. A two weeks supply of steel and modules are shown but only with a one week supply of scintillator in barrels. The steel octagon planes are assembled on steel I-beams called strongbacks.

\footnotetext{
${ }^{5}$ A strongback is made from heavy I-beams to provide support to the steel planes during assembly and lifting.
} 


\subsection{ASSEMBLY AND SUPPORT OF THE ACTIVE DETECTOR PLANE}

This section describes the assembly of the steel absorber plane and the liquid scintillator active detector plane. The assembly of the steel plane on the horizontal strongback is described first. Next, the active detector plane is attached to the surface of the steel plane. The combined steel and active planes are lifted to the vertical position using the strongback as support with an overhead crane. The planes are placed on the support rails of the detector. The final part of this section describes filling the active layer with scintillator after the active plane is installed in the detector.

The steel plane is composed of 8 plates of steel, each $2 \mathrm{~m}$ wide and $8 \mathrm{~m}$ long. The assembly starts by laying 4 plates of steel on a strongback constructed of I-beams. The strongback is required to keep the planes flat during the welding stages. Four more planes are placed over the first four planes at $90^{\circ}$ to the first layer, pressed flat, and welded.

The plates are welded together using a plugweld method. Holes in the top steel layer expose the bottom. By starting the welds on the bottom layer and filling the holes, all 8 plates of steel are joined together in a single octagon shape. The welded octagon will be theoretically flatter than the individual plates of steel.

The liquid scintillator detector only needs three assembly stations, shown previously in Fig. 19, because of its simple design. The first two stations are used for assembling the steel planes. The first station is for layout of the steel plates, pressing, and plug welding. The brackets and a support shelf, also described in Section 3.2, are welded to the steel plane at the second station. The last station is used to attach the liquid scintillator active detector modules to the steel plane. 
Figure 20 shows four edges of the steel plane covered by the steel strip shelf. The 8 strips are made from $2 \mathrm{~cm}$ square bar and are cut approximately $130 \mathrm{~cm}$ long. The strips are placed with their inner edges $4 \mathrm{~cm}$ from the outer edge of the steel. The shelf is needed along the outside edges of the active detector modules that require support.

The steel strips do not combine to make a continuous shelf. Along the bottom and vertical edges, the strips are positioned at least $6 \mathrm{~cm}$ from the shelf corners. This allows the two extrusions that extend past the shelf corners to rest against the shelf and their adjacent modules. If the space is not provided, the corner of one extrusion module would rest on the corner of the shelf and a gap would occur between this module and the adjacent module. The strips are welded in placed by $5 \mathrm{~cm}$ long- $5 \mathrm{~mm}$ fillet welds that are spaced $15 \mathrm{~cm}$ apart.

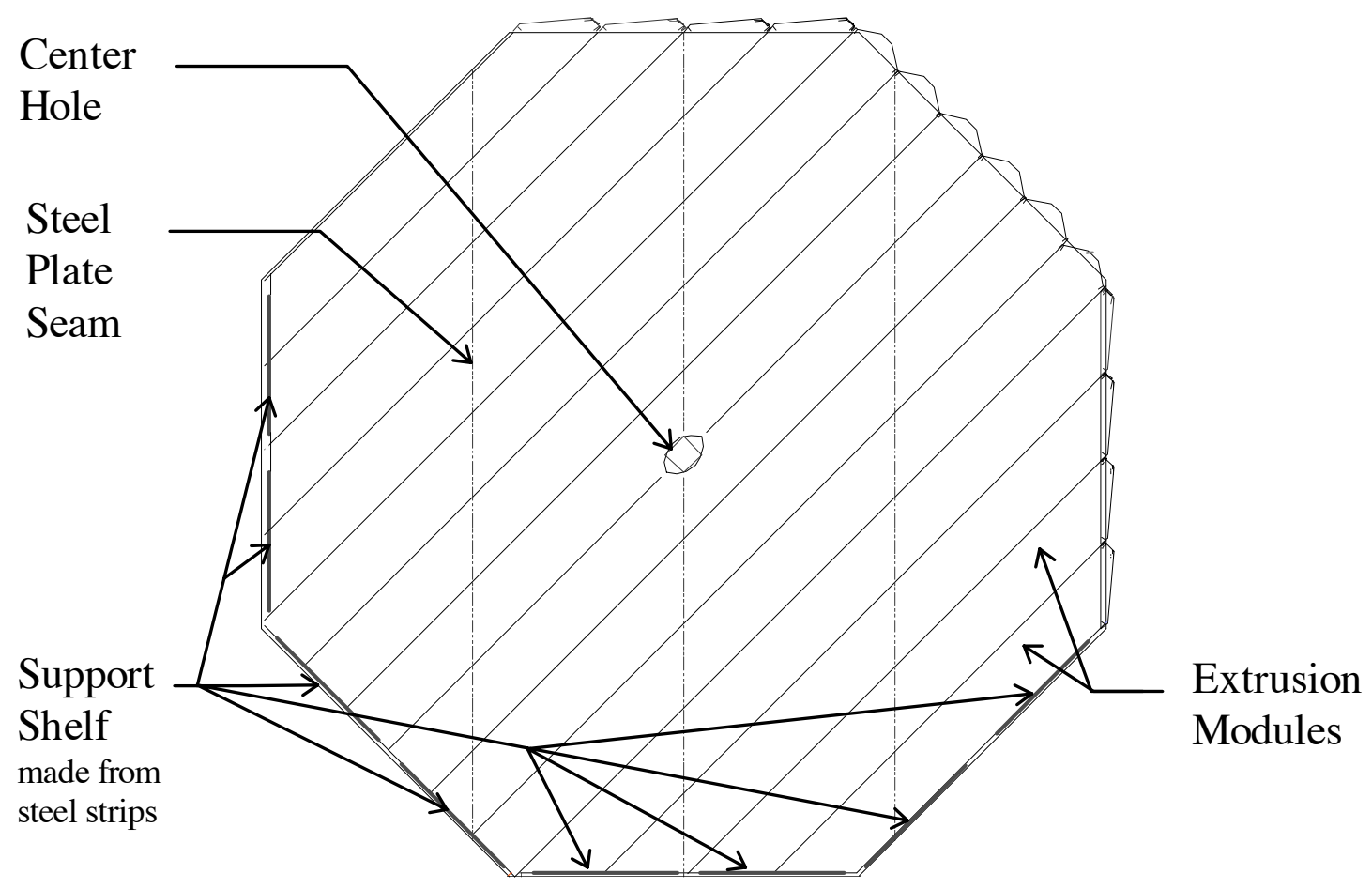

Figure 20. Top view of steel plane on strongback showing the support shelf and layout of extrusion modules. The shortest module is installed first against the shelf followed successively by the remainder. 
The active detector plane is laid out by placing the extrusion modules on top of the steel plane. Double sided tape is placed on the steel plane's surface to hold the modules in place. The shortest module is placed first on the steel plane against one edge of the support shelf as shown in Fig. 20. The bottom endcap is in contact with the steel strip on the bottom of the steel plane. The side of the extrusion is placed against the steel strip on the $45^{\circ}$ angle side of the plane. The manifold of the extrusion module is oriented towards the top. The next sized module is placed on the plane adjacent to the first module in the same orientation. The process continues placing the proper sized module in the proper place until the plane is filled with 14 modules. The two modules with the semi-circular indentations face each other in the middle of the plane.

Besides creating a solid surface for assembling the plane, the strongback provides the rigidity for lifting the completed assembly to the vertical position. The double-sided tape holds the modules on the absorber during the lifting stage. Once in place, the modules are contained by the steel planes and the support shelf.

The detector planes are placed on the steel support structure, creating a "hanging file", described in Section 3.1. The planes are held in place by axial bolts in four corners of the octagon. The bolts are required for spacing rather than structural support. A collar is positioned between planes and around the center hole to also create proper spacing.

The extrusion modules are not filled with scintillator until the planes have been installed in the detector. Each active plane is filled with liquid scintillator from a plumbing distribution system installed along the walls of the hall. The system consists of a central mixing tank and pump. A tube is plumbed on both sides of the detector cavern. A shutoff valve is installed in the tube every 5-10 meters. A moveable filling wand is attached to the valve closest to the active layer to be filled. 
The manifold in each module contains a fill port and a vent port. Each module in the plane is filled individually and each module remains an independent vessel for the scintillator. The fill and vent ports are plugged after filling. The pressure inside the detector is lower and leaks are not as likely to occur at the manifold because each module is independent. If the module is to be filled higher than the vent port, a plug is installed in the vent port before filling the remaining volume in the module.

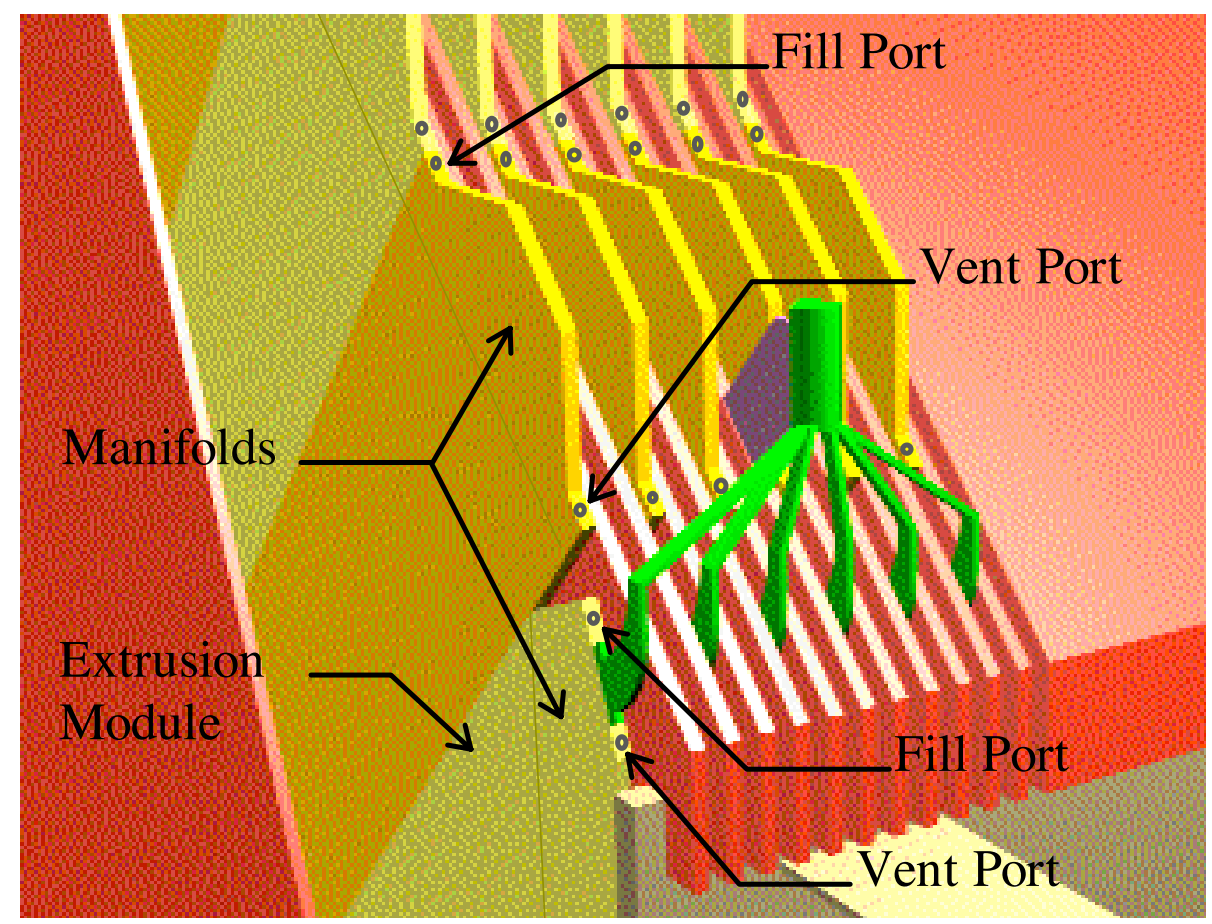

Figure 21. Close up view of fill ports and vent ports on two types of manifolds. 


\section{ANALYSIS AND TESTING OF ACTIVE DETECTOR MODULES}

The internal pressures in the extrusion modules vary with location in the detector. At the top of the detector, the internal pressure is $0.0 \mathrm{kPa}$ and the loading is $0.0 \mathrm{~N} / \mathrm{cm}$. The internal pressure at the bottom depends on which module is analyzed. Since each module is independently filled, the head pressure of each module is different. The internal pressure varies from $28.2 \mathrm{kPa}(4.1 \mathrm{psi})$ at the bottom of the shortest module to $52.4 \mathrm{kPa}(7.6 \mathrm{psi})$ at the bottom of the longest module. The internal pressure is applied on all internal surfaces including side walls. Refer to Section 9.3, Appendix C for calculations.

Internal normal forces created by the internal pressures acts along the internal surfaces of the modules. The internal surface of the shortest module parallel to its thickness is $1.7 \mathrm{~cm}$ multiplied by the length of the module ${ }^{6}$. The internal normal force at the bottom of the shortest module is $4.8 \mathrm{~N} / \mathrm{cm}(2.7 \mathrm{lbf} / \mathrm{in})$ along that surface.

The external loading on the modules also varies by location. The loading is dependent on the mass of the extrusions and scintillator above the location being analyzed. The downward pressure at the bottom of the detector is $70.6 \mathrm{kPa}$. The downward external load on the $2 \mathrm{~cm}$ thick active detector is $14.1 \mathrm{~N} /$ horizontal-cm. Since the modules are installed in the detector at $45^{\circ}$, the normal external load on the lowest module is $7.1 \mathrm{~N} / \mathrm{cm}$ (4.0 lbf/in) along the edge of the module.

The net normal load experienced by the module walls is the difference between the external normal load and the internal normal force. The internal normal force of the

\footnotetext{
${ }^{6}$ This is equal to the thickness of the module minus twice the external wall thickness.
} 
shortest module is $4.8 \mathrm{~N} / \mathrm{cm}(2.7 \mathrm{lbf} / \mathrm{in})$. The net normal load at the bottom of the shortest module is $2.3 \mathrm{~N} / \mathrm{cm}(1.3 \mathrm{lbf} / \mathrm{in})$.

The aging characteristics of the extrusion modules are unclear. No support structure is included between adjacent modules. One module lies against the adjacent lower module. Calculations show that the lowest module in the detector will be able to support those modules above when filled with liquid scintillator.

Plastics tend to creep. Preliminary accelerated tests were performed on an available extrusion to determine if the creep would be within limits for the run duration of the detector. A polypropylene extrusion, $1.5 \mathrm{~m}$ long by $0.5 \mathrm{~m}$ wide and $1.623 \mathrm{~cm}$ thick, was subjected to an internal pressure of $280 \mathrm{kPa}$ (40 psi) for a period of 7 weeks. The changes in the cell thickness are documented in Table 2. The first cell in the test extrusion is 2.5 $\mathrm{cm}$ wide so it expands more than an internal square-edged cell that is $1.6 \mathrm{~cm}$ wide. Figure 22 presents the data in a graphical format.

\begin{tabular}{|c|c|c|c|c|}
\hline Days & $\begin{array}{c}\text { Sq. Edge Cell Thickness } \\
(\mathrm{cm})\end{array}$ & First Cell Thickness $(\mathrm{cm})$ & Pressure (kPa) & Overall Width (cm) \\
\hline$<0$ & 1.623 & 1.623 & 0.000 & 37.795 \\
0.01 & 1.727 & 1.918 & 275.790 & 37.617 \\
0.1 & 1.765 & 1.956 & 275.790 & 37.617 \\
0.25 & 1.778 & 1.974 & 268.896 & 37.617 \\
0.5 & 1.786 & 1.986 & 268.896 & 37.617 \\
1 & 1.791 & 1.994 & 268.896 & 37.617 \\
1.5 & 1.798 & 1.999 & 275.790 & 37.617 \\
2 & 1.801 & 2.007 & 268.896 & 37.465 \\
3 & 1.808 & 2.017 & 275.790 & 37.465 \\
9 & 1.829 & 2.035 & 275.790 & 37.465 \\
13 & 1.842 & 2.042 & 279.238 & 37.465 \\
17 & 1.852 & 2.047 & 275.790 & 37.465 \\
22 & 1.857 & 2.050 & 282.685 & 37.465 \\
26 & 1.862 & 2.057 & 275.790 & 37.465 \\
33 & 1.869 & 2.060 & 275.790 & 37.465 \\
40 & 1.872 & 2.060 & 275.790 & 37.465 \\
46 & 1.875 & 2.062 & 275.790 & 37.465 \\
49 & 1.875 & 2.062 & 275.790 & 37.465 \\
\hline
\end{tabular}

Table 2. Results from internal pressure tests of a polypropylene extrusion from 40 psi pressure over a seven week period. 


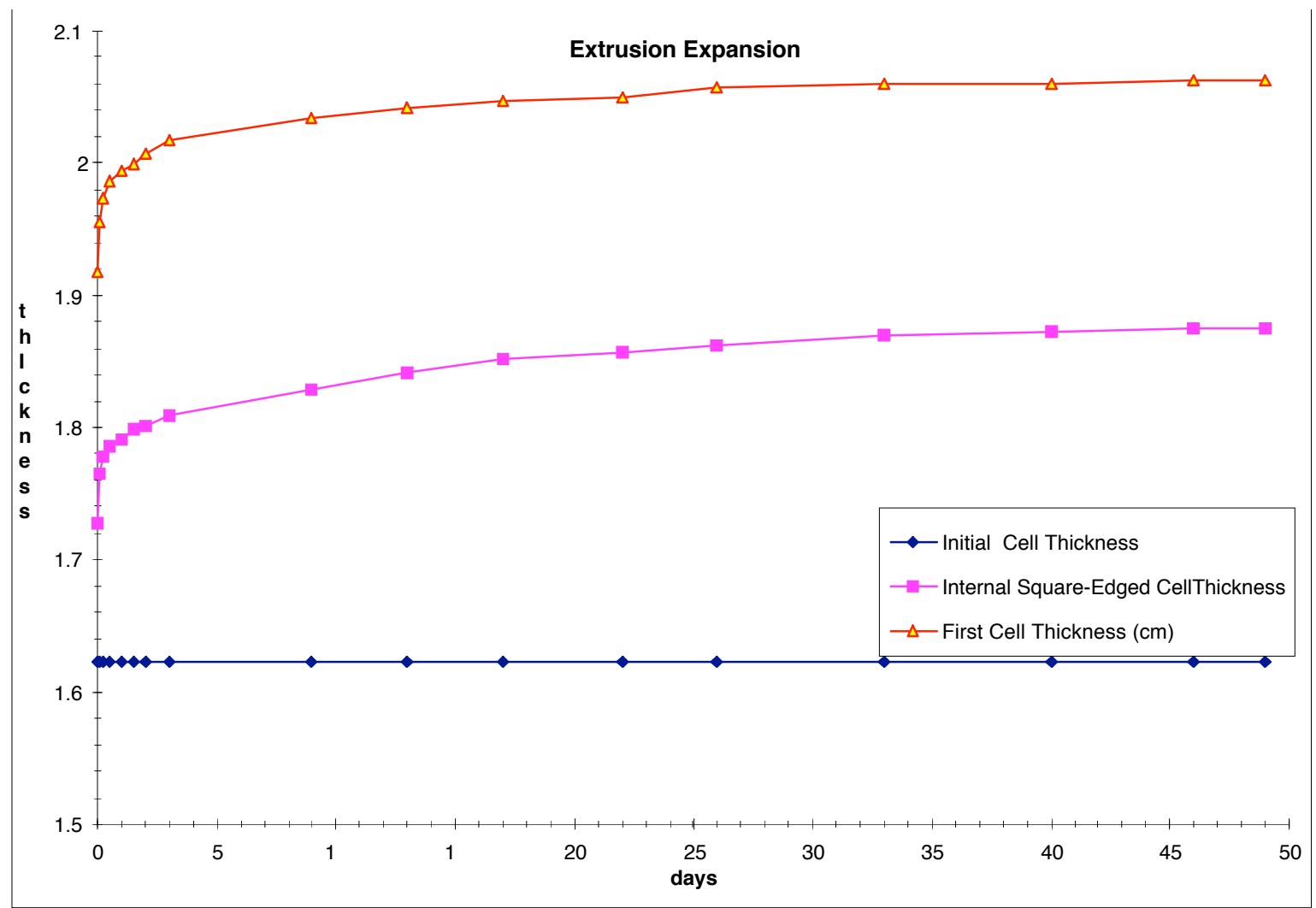

Figure 22. Graph of expansion of polypropylene extrusion from 40 psi internal pressure over a seven week period.

Another polypropylene extrusion was subjected to an external load test for 7 weeks. The extrusion was subjected to the internal pressures and external loads expected in the lowest extrusion. The internal pressure was simulated by filling the extrusion with water and adding a $6.4 \mathrm{~mm}(1 / 4$ ") diameter polyethylene tube filled with water to a height of $2.9 \mathrm{~m}$ (9.5 ft). The external pressure was simulated by adding 2 layers of lead bricks along the $45^{\circ}$ edge of the extrusion. After the 7 week test, there was no apparent change in the width of the extrusion. The thickness of the extrusion also had no apparent change. The only noticeable change in the experiment was a drop of $0.25 \mathrm{~m}$ (10 in) in the polyethylene tube water level. 


\section{CONCLUSION}

\subsection{REVIEW}

This thesis has presented a design for a liquid scintillator particle detector that is superior to previous liquid scintillator detectors. Chapter 2 defined the requirements for a workable liquid scintillator detector including total mass, cell width, and cost. Chapter 3 presented the design of the detector based on these requirements. The size and shape of the components such as extrusion modules were optimized by a mix of detector geometry, cost, and other available components like photo-detectors. The extrusion modules include an endcap, top manifold, optical fibers, and in some cases a bypass.

Chapter 4 described the manufacturing process of the extrusion modules. The modules are completely sealed units. An endcap is applied to the bottom. A manifold is attached to the top of the module. The manifold allows for filling the modules with liquid scintillator and is the termination point for the fibers inside the modules.

The assembly of the modules in the detector was discussed in Chapter 5. The modules are attached to the steel absorber planes. The planes are assembled into the detector. An optical light guide harness is attached to the manifold of each module, which in turn attaches to a photo-detector.

Chapter 6 discussed the life testing of the modules. The modules are subjected to both internal hydrostatic pressure and external forces from the modules above. 


\subsection{CONCLUSIONS}

The focus of this thesis is the design for a liquid scintillator particle detector. The objective of a superior design is to take what is currently available and add new techniques to improve on past designs. This design eliminates scintillator leakage by designing the fibers to exit only from the top of the modules. The bottom of the modules are completely sealed.

Contributions to the liquid scintillator detector design includes the layout of the active detector plane, the endcap design, the design of the manifold, and the layout of the optical light guide harness. In addition, some work was done on accelerated pressure testing and external loading of the plastic extrusions over a period of time.

The layout of the active detector plane was optimized to the number of channels in a photo-detector and the octagonal shape of the detector. The number of channels in a photo-detector was used to determine the best number of cells in a module. The geometry of the detector was used to determine the best number of modules in a plane.

A method of sealing the extrusion modules was developed using a molten endcap. The procedure was partially optimized for the rate of heating the endcap and the application temperature. The endcap must also be applied for the proper length of time before the mold is removed.

The manifold design funnels the fibers to a manageable area for attachment to the light guide harness. The manifold creates a sealed self-contained module by sealing the fibers and the top of the module. The manifold also allows for filling the modules with liquid scintillator. 
The optical harness layout is divided between 6 extrusion modules. One end of the harness is attached to the photo-detector. The other end of the light guide is divided between 6 terminal ends that mate with the optical fibers in 6 module manifolds.

Another area of ongoing research is the testing of plastic extrusions under various loads. Extrusions are subjected to both internal pressures and external loads to determine the effects on the extrusions over a long period. The internal pressure used in the pressure tests is 4 times the maximum anticipated pressure for the detector. The results of the tests will determine if additional support is required for the extrusion modules.

\subsection{RECOMMENDATION FOR FUTURE WORK}

Several areas pertaining to the design of a liquid scintillator detector need further research. These include continued accelerated testing of the extrusion modules, refining the manifold and bypass designs, possible changes in the plane layout due to external design changes, and refinement of the light guide harness.

The plastic extrusions require continued accelerated pressure testing to ensure that they have a life span that is longer than the life span of the detector ${ }^{7}$. This testing will examine the endcap attachment, the manifold attachment, and the bypass attachment.

The manifold design can be refined to eliminate the attaching side cover after the fibers are installed. Thus, the manifold would have only one open end at the end that attaches to the extrusion. The change embodies adding a separate guide to align the fibers to the orientation of the manifold holes. The guide and the fibers as a whole can be inserted inside the manifold. The fibers would automatically be threaded through the manifold

\footnotetext{
${ }^{7}$ Accelerated tests are not capable of exactly simulating actual aging.
} 
holes without access through one side. This design eliminates later welding of the side cover.

A prototype bypass needs to be fabricated and attached to the extrusion. The bypass design can benefit from additional refinements. Better methods of installing the fibers into the bypass and the bypass into the extrusion can probably be realized.

The layout of the extrusion modules is based on the octagonal shape of the detector and the current maximum extrusion width. New methods of extruding may allow wider extrusions. Changes in recommended cell widths and the number of channels in a photodetector may also affect the layout. Hanging the detector planes allows greater flexibility to the layout of the active detector plane.

The layout of light guide harness is related to the channels in a photo-detector. As the number of channels increases, the light guide harness will also change to accommodate more active detector planes. Changes in cell width will also affect the number of modules connected to one photo-detector.

Even though improvements can be made to the design of the liquid scintillator detector presented in this thesis, the design that exists is viable. With the exception of the continued testing of the extrusion modules, most of the future recommendations are unessential refinements. Several cost savings techniques have been developed; for example, the application procedure of the endcap. If the detector were built today, it is likely that it would function as intended. 


\section{REFERENCES}

Aglietta, M. et al., 1995, "The LVD in the Gran Sasso (Abstract)," (Inspec database), Nuovo Cimento C, vol. 18 C, ser. 1, no. 6, pp. 629-45.

Barnett, R. M., et al, 1996, "Review of Particle Physics," Physical Review D (Particles and Fields), vol. 54, no. 1, pt. 1, pp. 85-89.

Beier, E. W., 1995, “The Problems with Solar Neutrinos,” AIP Conference Proceedings, no. 334, pp. 290-304.

Cardall, C. Y. and Fuller, G. M., 1996, "Can a 'Natural' Three-Generation Neutrino Mixing Scheme Satisfy Everything," Physical Review D (Particles, Fields, Gravitation, and Cosmology), vol. 53, no. 8, pp. 4421-9.

CHORUS Collaboration, 1996, World Wide Web, http://choruswww.cern.ch

COSMOS Collaboration, 1996, World Wide Web, http://pooh.physics.lsa.umich.edu/ www/e803/e803.html

Davis, R., 1994, "A Review of the Homestake Solar Neutrino Experiment," Progress in Particle and Nuclear Physics, vol. 32, pp. 13-31.

Drexlin, G., 1994, "KARMEN: Precision Test of the Standard Model with Neutrinos from Muon and Pion Decay," Progress in Particle and Nuclear Physics, vol. 32, pp. 374-379.

Fermilab, 1996, "The Search for Neutrino Mass," (Brochure), Fermilab, A Department of Energy National Laboratory, Batavia, IL.

Gallagher, H.M., 1996, "Neutrino Oscillation Searches with the Soudan 2 Detector," Ph. D. Thesis, University of Minnesota, Minneapolis, MN. 
Gomez-Cadenas, J. J. and Gonzalez-Garcia, M .C., 1996, "Future nu/sub tau Oscillation Experiments and Present Data," Zeitschrift fur Physik C (Particle and Fields), vol. 71, no. 3, pp. 443-54.

KARMEN collaboration, 1996, "The KARMEN Detector" and "The Search for Neutrino Oscillation,” World Wide Web http://www-ik1.fzk.de/www/karmen/karmen_e.html

Laveder, M., 1995, “The NOMAD Experiment: Status Report," Nuclear Physics B Proceeding Supplements, vol. 48, pp. 188-92.

LSND Collaboration, 1996, World Wide Web, http://darkmatter.linfield.edu/ research/neutrino

MINOS Collaboration, 1996, World Wide Web, http://www.hep.umn.edu/minos/

Nakumura, K., "Solar Neutrinos," "Review of Particle Physics," Physical Review D (Particles and Fields), vol. 54, no. 1, pt. 1, pp. 275-79.

NOMAD Collaboration, 1996, World Wide Web, http://nomadinfo.cern.ch

Patzak, T., 1995, "Design and Performance of the CHORUS Detector," Proceedings of the 27th International Conference on High Energy Physics, pt. 2, vol. XIX+1455, pp. 1367-9, vol. 2.

Perkins, D. H., 1987, "Introduction to High Energy Physics," 3rd edition, Mento Park, CA., Addison-Wesley Publishing Co.

Pless, I. A. et al., 1993, "Status of the LVD Experiment at Gran Sasso Laboratory," AIP Conference Proceedings, no. 272, pt. 2, pp. 1242-5.

Pontecorvo, B., 1982, “The Infancy and Youth of Neutrino Physics," Journal de Physique Colloque, vol. 43, no. C-8, pp. C8/ 221-36. 
Reines, F., 1994, "Forty Years of Neutrino Physics," Progress in Particle and Nuclear Physics, vol. 32, pp. 1-12.

Shrock, R. E., 1996, "Neutrinos," "Review of Particle Physics," Physical Review D (Particles and Fields), vol. 54, no. 1, pt. 1, pp. 275-79.

Zeitnitz, B., 1994, "KARMEN: Neutrino Physics at ISIS," Progress in Particle and Nuclear Physics, vol. 32, pp. 351-73. 


\section{APPENDICES}

\subsection{APPENDIX A}

\section{CALCULATIONS FOR COMPONENTS AND LAYOUT}

Each extrusion module is self-contained and consists of the following parts:
1) Extrusion
2) Endcap
3) Manifold
4) Manifold cover
5) Optical Fibers

Requirements for extrusion module layout of the detector with center hole

1) Symmetrical module layout across octagon.

2) Even number of extrusions

Extrusions must fill the octagon plane and fit within the shape of the octagon shown in Fig. 23 (at the right). The extrusions are supported by a shelf along four of the lower edges of the octagon. The shelf is made from 8 steel strips -2 on each edge.

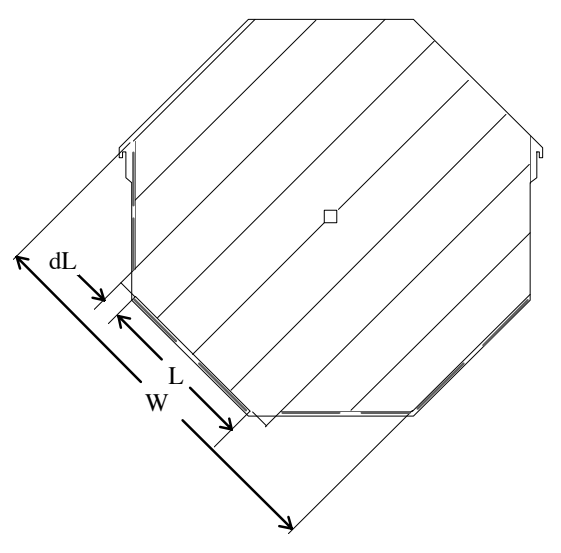

Figure 23. Layout of extrusion

The available width for all extrusions is the overall width minus twice the width required for the shelf installation

where: $\quad \mathrm{W}=800 \mathrm{~cm}-2 *$ shelf installation width and: $\quad 2 \mathrm{~cm}<$ shelf installation width $<6 \mathrm{~cm}$ All edges ' $L$ ' are equal in length and the corners of the octagon meet at $45^{\circ}$. 
For an octagon, the ratio

$$
\frac{L}{W}=\frac{1}{2 * \cos 45^{\circ}+1}
$$

Assume required width for installation of the shelf is $4 \mathrm{~cm}$. $(2 \mathrm{~cm}$ shelf width $+2 \mathrm{~cm}$ in from the edge of the octagon) $=>$

$$
\mathrm{W}=792 \mathrm{~cm} . \quad \mathrm{L}=328 \mathrm{~cm} .
$$

Table 3 shows the effects of varying the number of extrusion modules. The first column lists the total number of modules (\#W) per plane. The first five elements in the first row lists the number of extrusions (\#L) that may possibly fit across ' $L$ '. Refer also to Fig. 23.

Definition of variables in Table 3:

$\begin{array}{ll}\text { '\#L' } & \text { nearest number of extrusions across edge ' } \mathrm{L} \text { ' } \\ \text { '\#W' } & \text { number of extrusions across width ' } \mathrm{W} \text { ' } \\ \text { 'delta' } & \text { difference between optimum } \mathrm{L} / \mathrm{W} \text { ratio and the ratio } \# \mathrm{~L} / \# \mathrm{~W} \\ \text { 'EW' } & \text { width of each extrusion } \\ \text { 'EWL' } & \text { width of \#L extrusions } \\ \text { 'dL' } & .5 * \mid \mathrm{EWL}-\mathrm{Ll} \text { (distance from shelf corner to nearest extrusion seam) }\end{array}$

\begin{tabular}{|c|c|c|c|c|c|c|c|c|c|c|}
\hline \#W & $\# \mathrm{~L}$ & 2 & 4 & 6 & 8 & 10 & delta & $\begin{array}{c}E W \\
(\mathrm{~cm})\end{array}$ & $\begin{array}{c}E W L \\
(\mathrm{~cm})\end{array}$ & $\begin{array}{r}d L \\
(\mathrm{~cm})\end{array}$ \\
\hline 8 & & .25 & .50 & & & & .0858 & 99.0 & & \\
\hline 10 & & & .40 & .60 & & & .0142 & 79.2 & 316.8 & 5.6 \\
\hline 12 & & & .333 & .50 & & & .0812 & 66.0 & & \\
\hline 14 & & & .286 & .429 & & & .0145 & 56.57 & 339.4 & 5.7 \\
\hline 16 & & & & .375 & .50 & & .0392 & 49.5 & & \\
\hline 18 & & & & .333 & .444 & & .0298 & 44.0 & & \\
\hline 20 & & & & & .40 & .50 & .0142 & 39.6 & 316.8 & 5.6 \\
\hline
\end{tabular}

The number of extrusions that best fit the octagon shape has the smallest delta.

$\left|0.4142-\frac{\# L}{\# W}\right|=$ delta $\quad$-the smallest delta is based on the ratio \#L/\#W in bold type.

Table 3. Ratios of the number of extrusions across ' $L$ ' and total number of extrusions across ' $W$ '. The best choices have the smallest deltas and ' $d L s$ '.

Three ratios show approximately the same ' $\mathrm{dL}$ '. The three choices compare as follows: 

1) 10 modules per plane
$79.2 \mathrm{~cm}$ wide
may be too wide to extrude
2) 14 modules per plane
$56.6 \mathrm{~cm}$ wide
$\mathrm{OK}$
3) 20 modules per plane
$39.6 \mathrm{~cm}$ wide
too many pieces

Fourteen extrusion modules appears to be the best choice.

To complete the number of modules derivation, a check is performed to see if any extrusion extends past the bottom. The portion of the extrusion that extends below the shelf in Fig. 24 is equal to $\mathrm{dL} * \cos 45^{\circ}$. The inside of the shelf is $4 \mathrm{~cm}$ from the edge.

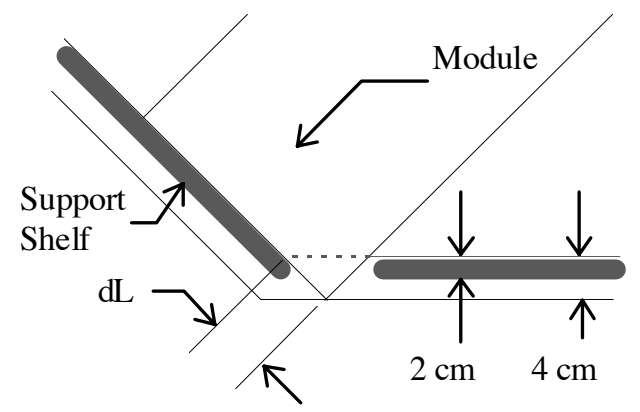

Figure 24. Corner of octagon with module and support shelf.

Only a small amount extends past the bottom edge. The endcap of the module has enough material to allow trimming the corner this small amount.

\subsubsection{Calculate number of cells}

The number of cells in one extrusion depend on the number of channels in a photodetector and the number of extrusion modules.

Cells $=$ channels/extrusions

Each photo-detector will have 156 channels.
a) 5 extrusions
$156 / 5=31.2$ cell
$\Rightarrow \quad 31$ cells
b) 6 extrusions
$156 / 6=26.0$ cell
$\Rightarrow \quad 26$ cells
c) 7 extrusions
$156 / 7=22.3$ cell
$\Rightarrow \quad 22$ cells 


\subsubsection{Calculate cell width}

The extrusion width is $56.57 \mathrm{~cm}$.
a) 5 extrusions
31 cells
$56.57 / 31=1.82 \mathrm{~cm} /$ cell
b) 6 extrusions
26 cells
$56.57 / 26=2.17 \mathrm{~cm} / \mathrm{cell}$
c) 7 extrusions
22 cells
$56.57 / 22=2.57 \mathrm{~cm} /$ cell

\subsubsection{Alternate method}

Specify cell width to be $2 \mathrm{~cm}$. Find the number of channels per photo-detector.

Calculate number of cells.

$56.57 / 2=28.28=>28$ cells per modules

Calculate number of channels per photo-detector.
a) 5 extrusions
28 cells
$28 * 5=140$ channels/photo-detector
b) 6 extrusions
28 cells
$28 * 6=168$ channels/photo-detector

The maximum number of channels is 156 channels. Only 5 extrusions can be used for each photo-detector using this method. 


\subsection{APPENDIX B}

\section{CALCULATIONS -- DIMENSIONS, AREAS, VOLUMES, AND MASS}

Parameters for the detector
a) ' $\mathrm{SW}$ ' 8 meter wide octagon
b) ' $\mathrm{D}$ ' $\quad 30 \mathrm{~cm}$ diameter hole in the center
c) 'TM' Total mass - 10,000 metric tons $(22,000,000 \mathrm{lbm})$
d) ' $\mathrm{ST}$ ' $2 \mathrm{~cm}$ thick steel planes
e) 'SD' steel density $7865 \mathrm{~kg} / \mathrm{m}^{3}$
f) 'ET' $2 \mathrm{~cm}$ thick plastic extrusions
g) 'ED' plastic density $900 \mathrm{~kg} / \mathrm{m}^{3}$
h) 'SCD' Scintillator density $850 \mathrm{~kg} / \mathrm{m}^{3}$
j) 'AS' $1 \mathrm{~cm}$ extra airspace between steel planes
k) 'NE' 14 extrusions per plane
l) 'EW' $56.57 \mathrm{~cm}$ extrusion width
m) 'NC' 26 cells per extrusion
n) ' $\mathrm{CW}$ ' $2.17 \mathrm{~cm}$ cell width
o) 'OW' extrusion outside wall thickness $\quad-1.5 \mathrm{~mm}$ thick
p) 'IW' extrusion inside wall thickness $\quad-1.0 \mathrm{~mm}$ thick
q) 'W' $792 \mathrm{~cm}$ octagon width excluding allowance for shelves
r) ' $L$ ' $328 \mathrm{~cm}$ wide on one side of octagon
s) 'NEL' 6 extrusions across L
t) 'SL' 1.30 meter steel shelf strips (8 per plane- $2 \mathrm{~cm} \times 2 \mathrm{~cm}$ cross-section)
u) 'FDI' $1 \mathrm{~mm}$ dia. optical fiber per cell
v) 'FD' fiber density $950 \mathrm{~kg} / \mathrm{m}^{3}$

This appendix is divided into five sections:

1) Calculation of area, volume, and mass for the steel in the plane and shelf.

2) Calculation of area, volume, and mass for the extrusions.

3) Calculation of volume and mass for the fibers.

4) Calculation of volume and mass for the scintillator.

5) Calculation of the total number of planes and the length of the detector. 


\subsubsection{STEEL OCTAGON AREA, VOLUME, AND MASS}

Refer to diagram of octagon in Fig. 25 and parameters listed in Section 9.2.

Length of each side of octagon 'OL'

$$
\begin{aligned}
& \mathrm{OL}+2^{*} \mathrm{OL} * \cos 45^{\circ}=\mathrm{SW} \\
& =>\mathrm{SW} /(1+2 * \cos 45) \quad=\mathrm{OL} \\
& 8 /(1+\cos 45) \quad=\text { OL } \quad \text { Length of steel side }=3.3137 \mathrm{~m}
\end{aligned}
$$

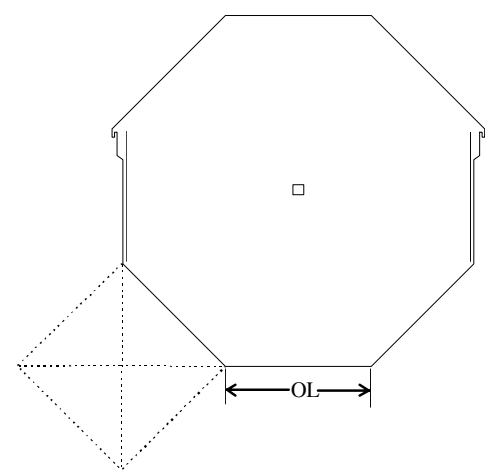

Figure 25. Octagon Layout

Length of steel side $=3.3137 \mathrm{~m}$

Area of Steel Plane with $30 \mathrm{~cm}$ dia hole 'OA'

$$
\begin{aligned}
& \mathrm{SW}^{2}-\mathrm{OL}^{2}-\pi * \mathrm{D}^{2} / 4=\mathrm{OA} \\
& 8^{2}-3.3137^{2}-\pi * .30^{2} / 4=\mathrm{OA}
\end{aligned}
$$

Octagon Area

$$
=52.95 \mathrm{~m}^{2}
$$

Volume of Steel Octagon Plane

'OV'

$$
\begin{array}{ll}
\mathrm{OA} * \mathrm{ST} & =\mathrm{OV} \\
52.95 * .02 & =\mathrm{OV}
\end{array}
$$

Octagon Volume $=1.059 \mathrm{~m}^{3}$

Volume of Steel Shelf 'SV'

$$
\begin{array}{ll}
8 * \mathrm{SL}^{*} .02^{2} & =\mathrm{SV} \\
8 * 1.3 * .02^{2} & =\mathrm{SV}
\end{array}
$$

Shelf Volume $=0.0042 \mathrm{~m}^{3}$

Mass of Steel Plane and Steel Shelf 'MS'

$$
\begin{array}{ll}
(\mathrm{OV}+\mathrm{SV}) * \mathrm{SD} & =\mathrm{MS} \\
(1.059+0.0042) * 7865 & =\mathrm{MS}
\end{array}
$$

Steel mass $=8362.0 \mathrm{~kg}$ 


\subsubsection{EXTRUSION AREA, VOLUME, AND MASS}

Cross-Sectional Area Of Extrusion Profile 'EA'

$$
\begin{array}{ll}
2 *(\mathrm{EW}+(\mathrm{ET}-2 * \mathrm{OW})) * \mathrm{OW}+(\mathrm{NC}-1) *(\mathrm{ET}-2 * \mathrm{OW}) * \mathrm{IW} & =\mathrm{EA} \\
2 *(56.57+1.7) * .15+25 * 1.7 * .10 & =\mathrm{EA}
\end{array}
$$

Extrusion Cross-section Area $\quad=21.731 \mathrm{~cm}^{2}$

Average Length of Extrusions (approx.) 'AL'

$$
\begin{aligned}
& \frac{N E L}{N E} * W+\frac{N E-N E L}{N E} * \frac{(W+L)}{2}=A L \\
& \frac{6}{14} * 7.92+\frac{8}{14} * \frac{(7.92+3.28)}{2}=A L \quad \text { Average Extrusion Length } \quad=6.595 \mathrm{~m}
\end{aligned}
$$

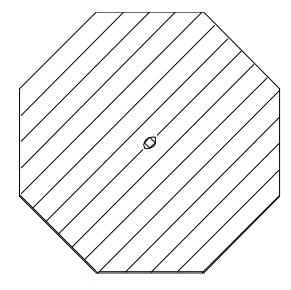

Volume of Extrusions Per Plane 'VE'

$$
\begin{aligned}
& \mathrm{NE} * \mathrm{EA} * \mathrm{AL} \quad=\mathrm{VE} \\
& 14 * 21.73 * 10^{-4} * 6.595=\mathrm{VE} \quad \text { Extrusion Volume }=0.2006 \mathrm{~m}^{3}
\end{aligned}
$$

Mass of Extrusions Per Plane 'ME'

$$
\begin{array}{lll}
\mathrm{VE} * \mathrm{ED} & =\mathrm{ME} & \\
0.2006 * 900 & =\mathrm{ME} & \text { Extrusion Mass }
\end{array}
$$

\subsubsection{OPTICAL FIBER--VOLUME AND MASS (within octagon area)}

The diameter of optical fiber is $1 \mathrm{~mm}$.

Volume of Fibers per Plane

$$
\begin{array}{ll}
\mathrm{NE} * \mathrm{NC} * \mathrm{AL}^{*} \pi *\left(\mathrm{FDI}^{2}\right) / 4 & =\mathrm{FV} \\
14 * 26 * 6.595 * \pi *\left(.001^{2}\right) / 4 & =\mathrm{FV}
\end{array}
$$

Mass of Fibers per Plane

'FM'
'FV'

Fiber Volume

$=0.0019 \mathrm{~m}^{3}$
Fiber Mass

$=1.80 \mathrm{~kg}$ 


\subsubsection{SCINTILLATOR VOLUME AND MASS}

Gross Volume of Scintillator per plane

$$
\begin{array}{ll}
\text { ross Volume of Scintillator per plane } & \mathrm{SCV} \\
\mathrm{W} * \mathrm{AL} * \mathrm{ET}-\mathrm{VE} & =\mathrm{SCV} \\
7.92 * 6.595 * .02-.2006=\mathrm{SCV} & =0.8440 \mathrm{~m}^{3}
\end{array}
$$

Remove the volume of scintillator vacated by the extrusion bypasses of the center hole (estimated at 1.5 times the area of the center hole times the extrusion thickness) 'SCVC'

$$
\begin{array}{ll}
1.5 * \pi * \mathrm{D}^{2} / 4 * \mathrm{ET} & =\mathrm{SCVC} \\
1.5 * \pi * .30^{2} / 4 * .02 & =\mathrm{SCVC}
\end{array}=0.0021 \mathrm{~m}^{3}
$$

Adjusted Volume of Scintillator per Plane 'ASCV' adjusted for center hole and fibers

$$
\begin{array}{ll}
\text { SCV-SCVC- FV } & =\text { ASCV } \\
.8440-.0021-.0019 & =\mathrm{ASCV}
\end{array}
$$

Convert to gallons

$$
.8400 * 39.37^{3}\left(\mathrm{in}^{3} / \mathrm{m}^{3}\right) / 231\left(\mathrm{in}^{3} / \mathrm{gal}\right)=
$$

'MSC'

Mass of Scintillator Per Plane

$$
\begin{array}{ll}
\mathrm{ASCV} * \mathrm{SCD} & =\mathrm{MSC} \\
.8400 * 850 & =\mathrm{MSC}
\end{array}
$$

$$
\begin{aligned}
& \text { Scintillator Volume } \quad \mathbf{= 0 . 8 4 0 0} \mathbf{m}^{\mathbf{3}} \\
& =840 \text { liters }
\end{aligned}
$$

$$
=221.9 \text { gals }
$$

\section{ENDCAP VOLUME AND MASS}

Calculations of the extrusion volume did not include the volume of the endcap. The density of the endcap and the density of the scintillator are almost the same. Addition of the mass and volume of the endcap reduces the mass and volume of the scintillator calculated above. Any change in total volume and mass will be negligible. 


\subsubsection{CALCULATIONS FOR TOTAL DETECTOR}

The mass of the detector is 10,000 metric tons $(22,000,000 \mathrm{lbm})$ ' $\mathrm{TM}$ '

The Mass of the Steel Plane

The Mass of the Extrusions

The Mass of the Fibers

The Mass of the Scintillator

$$
\begin{aligned}
\text { MS } & =8362.0 \mathrm{~kg} \\
\mathrm{ME} & =180.54 \mathrm{~kg} \\
\mathrm{FM} & =1.80 \mathrm{~kg} \\
\mathrm{MSC} & =714.0 \mathrm{~kg}
\end{aligned}
$$

\section{TOTAL NUMBER OF PLANES}

Mass of each Plane 'PM'

$$
\begin{array}{lll}
\mathrm{MS}+\mathrm{ME}+\mathrm{FM}+\mathrm{MSC} & =\mathrm{PM} \\
8362+180.54+1.8+714.0 & =\mathrm{PM}
\end{array} \quad \text { Mass of a Plane } \quad=9258.4 \mathrm{~kg}
$$

Number of Planes 'NP'

$$
\begin{array}{ll}
\mathrm{TM} / \mathrm{PM} & =\mathrm{NP} \\
10,000,000 / 9258.4 & =\mathrm{NP}
\end{array}
$$$$
\text { Number of Planes = } 1080 \text { Planes }
$$

\section{LENGTH OF DETECTOR 'L'}

The detector is divided into three sections with a 1 meter space between the sections and a 2 meter space on both ends.

Total Length ' $L$ '

$$
\begin{aligned}
& \mathrm{NP} *(\mathrm{ST}+\mathrm{ET}+\mathrm{AS})+2 *(2+1)=\mathrm{L} \\
& 1080 *(.02+.02+.01)+2 *(1+2)=\mathrm{L}
\end{aligned}
$$




\subsection{APPENDIX C}

\section{CALCULATIONS OF LOADS AND PRESSURES}

All load calculations are based on a density for plastic and scintillator of $900 \mathrm{~kg} / \mathrm{m}^{3}$

Acceleration due to gravity is $9.81 \mathrm{~m} / \mathrm{s}^{2}$. Assume there is no slip between extrusions.

Refer to Fig. 26 for calculations of loads and pressures at five places.

1) Bottom of lowest extrusion.

2) Top of lowest extrusion.

3) Fifth extrusion, 4 meters from bottom.

4) Bottom of fourth extrusion.

5) Bottom of tenth extrusion.

Conversion factor to psi -

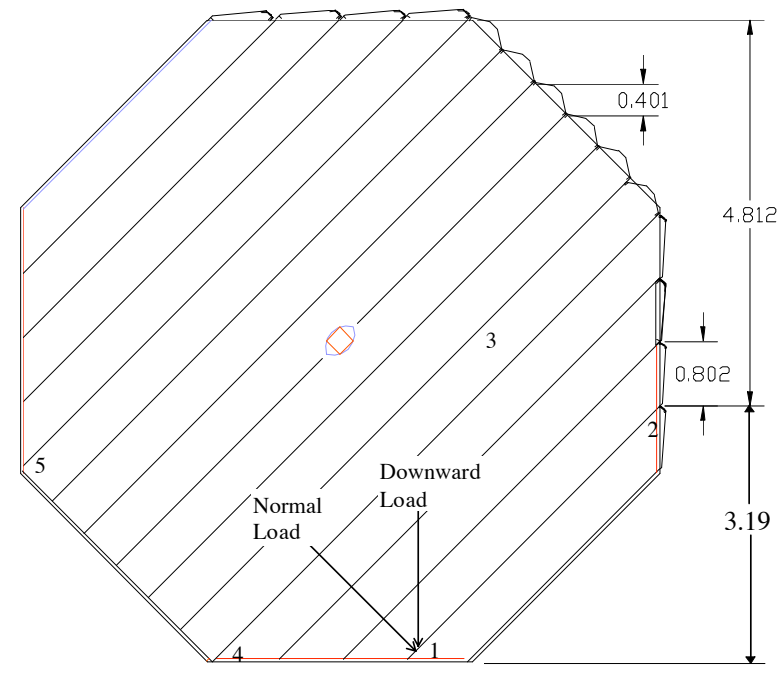

$$
\text { psi }=0.1450 * \mathrm{kPa}
$$

Figure 26. Layout of Extrusions

Press. $(\mathrm{kPa})=$ Density Scintillator $* \mathrm{Ht} * \mathrm{~g} / 10^{3}$

Internal Force/length $(\mathrm{N} / \mathrm{cm})=$ Press. $(\mathrm{kPa}) * 10^{3}\left(\mathrm{~N} / \mathrm{m}^{2}-\mathrm{kPa}\right) / 10^{4}\left(\mathrm{~cm}^{2} / \mathrm{m}^{2}\right) * 1.7(\mathrm{~cm}$ internal width)

Normal Force/length from internal pressure opposing Normal load $=\mathrm{kPa} * .170$ Linear Load $(\mathrm{N} / \mathrm{cm})=$ Press. $(\mathrm{kPa}) * 10^{3}\left(\mathrm{~N} / \mathrm{m}^{2}-\mathrm{kPa}\right) / 10^{4}\left(\mathrm{~cm}^{2} / \mathrm{m}^{2}\right) * 2(\mathrm{~cm}$ thick $)$

2 types - Downward load $/ \mathrm{cm}$ horizontal $=\mathrm{kPa} * .200$

Normal load $/ \mathrm{cm} 45^{\circ}$ axis $=\left(\right.$ Downward load $\left.* \cos 45^{\circ}\right) /\left(\mathrm{cm}\right.$ hor. $\left./ \cos 45^{\circ}\right)$

\section{Position 1}

Internal Pressure $=900 * 3.19 * 9.81 / 10^{3}$

Internal Force/unit length $=28.2 * 0.17$

Downward Press. $=900 * 8.00 * 9.81 / 10^{3}$

Downward Load $=70.6 * 0.20$

Normal Load $=14.1 *(\cos 45)^{2}$
Pressure \#1 $=28.2 \mathrm{kPa}(4.1 \mathrm{psi})$

Internal Force $=4.8 \mathrm{~N} / \mathrm{cm}(2.7 \mathrm{lb} / \mathrm{in})$

Down Press. $=70.6 \mathrm{kPa}(10.2 \mathrm{psi})$

Down Load $\quad=14.1 \mathrm{~N} / \mathrm{cm}(8.1 \mathrm{lb} / \mathrm{in})$

Normal load $=7.1 \mathrm{~N} / \mathrm{cm}(4.0 \mathrm{lb} / \mathrm{in})$ 


\section{Position 2}

Internal Pressure $=900 * 0.0 * 9.81 / 10^{3}$

Internal Force/unit length $=0.0 * 0.17$

Downward Press. $=900 * 2.5 * 9.81 / 10^{3}$

Downward Load $=22.1 * 0.20$

Normal Load $=4.4 *(\cos 45)^{2}$
Pressure \#2 $=0.0 \mathrm{kPa}(0.0 \mathrm{psi})$

Internal Force $=0.0 \mathrm{~N} / \mathrm{cm}(0.0 \mathrm{lb} / \mathrm{in})$

Down Press. $=22.1 \mathrm{kPa}(3.2 \mathrm{psi})$

Down Load $=4.4 \mathrm{~N} / \mathrm{cm}(2.5 \mathrm{lb} / \mathrm{in})$

Normal load $=2.2 \mathrm{~N} / \mathrm{cm}(1.3 \mathrm{lb} / \mathrm{in})$

\section{Position 3}

Internal Pressure $=900 * 2.0 * 9.81 / 10^{3}$

Internal Force/unit length $=17.6 * 0.17$

Downward Press. $=900 * 4.0 * 9.81 / 10^{3}$

Downward Load $=35.3 * 0.20$

Normal Load $=7.1 *(\cos 45)^{2}$

\section{Position 4}

Internal Pressure $=900 * 5.6 * 9.81 / 10^{3}$

Internal Force/unit length $=49.4 * 0.17$

Downward Press. $=900 * 8.0 * 9.81 / 10^{3}$

Downward Load $=70.6 * 0.2$

Normal Load $=14.1 *(\cos 45)^{2}$
Pressure \#3 = $17.6 \mathrm{kPa}(2.6 \mathrm{psi})$

Internal Force $=3.0 \mathrm{~N} / \mathrm{cm}(1.7 \mathrm{lb} / \mathrm{in})$

Down Press. $=35.3 \mathrm{kPa}(5.1 \mathrm{psi})$

Down Load $=7.1 \mathrm{~N} / \mathrm{cm}(4.0 \mathrm{lbf} / \mathrm{in}$

Normal load $=3.5 \mathrm{~N} / \mathrm{cm}(2.0 \mathrm{lb} / \mathrm{in})$
Pressure \#4 = $49.4 \mathrm{kPa}(7.2 \mathrm{psi})$

Internal Force $=8.4 \mathrm{~N} / \mathrm{cm}(4.8 \mathrm{lb} / \mathrm{in})$

Down Press. $=70.6 \mathrm{kPa}(10.2 \mathrm{psi})$

Down Load $=14.1 \mathrm{~N} / \mathrm{cm}(8.1 \mathrm{lb} / \mathrm{in})$

Normal load $=7.1 \mathrm{~N} / \mathrm{cm}(4.0 \mathrm{lb} / \mathrm{in})$

\section{Position 5}

Internal Pressure $=900 * 5.6 * 9.81 / 10^{3}$

Internal Force/unit length $=49.4 * 0.17$

Downward Press. $=900 * 3.2 * 9.81 / 10^{3}$

Downward Load $=28.2 * 0.2$

Normal Load $=5.6 *(\cos 45)^{2}$
Pressure \#5 = 49.4 $\mathrm{kPa}(7.2 \mathrm{psi})$

Internal Force $=8.4 \mathrm{~N} / \mathrm{cm}(4.8 \mathrm{lb} / \mathrm{in})$

Down Press. $=28.2 \mathrm{kPa}(4.08 \mathrm{psi})$

Down Load $\quad=5.6 \mathrm{~N} / \mathrm{cm}(3.2 \mathrm{lb} / \mathrm{in})$

Normal load $=2.8 \mathrm{~N} / \mathrm{cm}(1.6 \mathrm{lb} / \mathrm{in})$ 
Maximum Internal Pressure

Maximum Internal Force

Maximum Downward Load

Maximum Normal Load
$49.4 \mathrm{kPa}(7.2 \mathrm{psi})$

$8.4 \mathrm{~N} / \mathrm{cm}(4.8 \mathrm{lbf} / \mathrm{in})$

$14.1 \mathrm{~N} / \mathrm{cm}(8.05 \mathrm{lbf} / \mathrm{in})$

$7.1 \mathrm{~N} / \mathrm{cm}$ (4.02 lbf/in)

It is important to note that the Internal Normal Force is the result of the internal head pressure of the liquid. The internal pressure always acts normal to the internal surface. This directly opposes the External Normal Load.

Differences between the Internal Normal Force and the External Normal Load.

Position $1 \quad 7.1-4.8 \quad$ Difference $=2.3 \mathrm{~N} / \mathrm{cm}(1.30 \mathrm{lb} / \mathrm{in})$ External load is higher than internal force

Position 2 2.2 $-0.0 \quad$ Difference $=2.2 \mathrm{~N} / \mathrm{cm}(1.26 \mathrm{lb} / \mathrm{in})$ External load is higher than internal force

Position $3 \quad 3.5-3.0 \quad$ Difference $=0.5 \mathrm{~N} / \mathrm{cm}(0.3 \mathrm{lb} / \mathrm{in})$ External load is higher than internal force

Position 4 7.1-8.4 Difference $=-1.3 \mathrm{~N} / \mathrm{cm}(-0.8 \mathrm{lb} / \mathrm{in})$ Internal force is higher than external load

Position 5 2.8-8.4 Difference $=-5.6 \mathrm{~N} / \mathrm{cm}(-3.2 \mathrm{lb} / \mathrm{in})$ Internal force is higher than external load

These load calculations involve only internal forces created by the head pressure of liquid scintillator. The walls of the extrusions provide support of the loads in addition to the internal forces. The maximum difference between the internal forces and the external loads is only $2.3 \mathrm{~N} / \mathrm{cm}(1.3 \mathrm{lbs} / \mathrm{in})$. The walls are assumed to be able to support this difference. However, the results of experimental tests to confirm this assumption are not complete at the time of this thesis. It is possible that the that the walls could buckle due to the vertical loading of the extrusions. 


\subsection{APPENDIX D}

\section{CALCULATION OF STORAGE SPACE}

Refer to Figs. 27-30.

Storage Space for Major Components

1) Steel plates

2) Extrusions

3) Scintillator

assume $\mathbf{1 2 0}$ planes at a time

( 3 months supply at 2 planes per day)

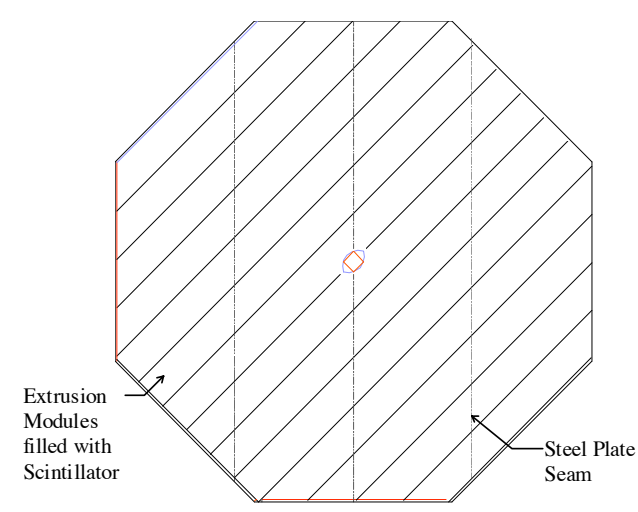

Figure 27. Reference for component storage space

Steel Plate Storage (120 planes- refer to Fig. 28)

8 steel plates required for each plane

a) 8 meters long

b) 2 meters wide

c) $1 \mathrm{~cm}$ thick

$$
\begin{aligned}
& 120 * 8=960 \text { plates } \\
& .01 * 960=9.6 \mathrm{~m}=>2 \text { stacks }
\end{aligned}
$$

Steel Storage Space $=8 \mathrm{~m} \mathrm{x} 4 \mathrm{~m} \times 4.8 \mathrm{~m}$

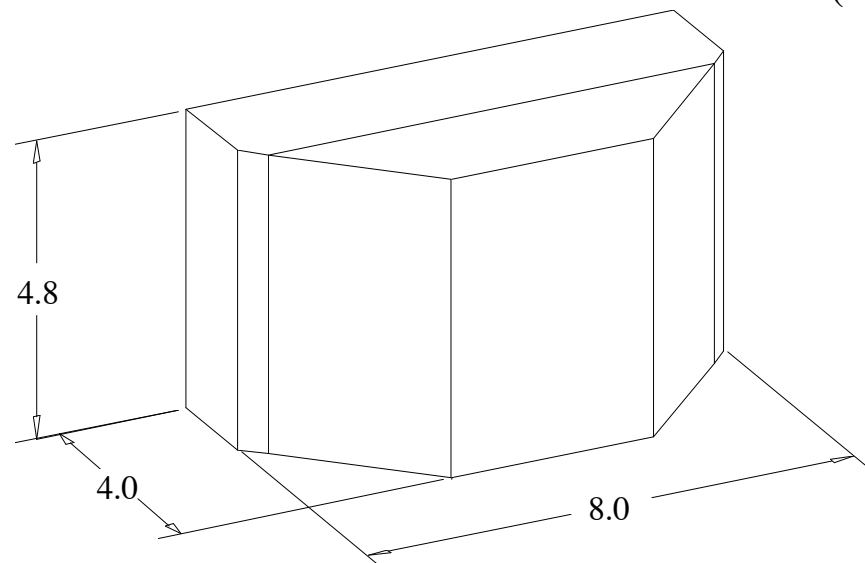

$\left(26.4^{\prime} \times 13.2^{\prime} \times 16^{\prime}\right)$

Figure 28. Steel storage 3 months supply 
Extrusion Storage (120 planes- refer to Fig. 29)

14 extrusions required per plane $-2 \mathrm{~cm}$ thick x $56.6 \mathrm{~cm}$ wide $120 * .02=2.4 \mathrm{~m}$ high
a) 8 stacks - 8.0 meters long
b) 2 stacks - 6.9 meters long
c) 2 stacks - 5.7 meters long
d) 2 stacks - 4.5 meters long
e) Total space required

Extrusion Storage $\quad 4$ ea. $\quad 8.0 \mathrm{~m} \times 1.2 \mathrm{~m}$ x $2.4 \mathrm{~m}$

1 ea. $\quad 6.9 \mathrm{~m} \times 1.2 \mathrm{~m} \times 2.4 \mathrm{~m}$

1 ea. $\quad 5.7 \mathrm{~m} \times 1.2 \mathrm{~m} \times 2.4 \mathrm{~m}$

1 ea. $\quad 4.5 \mathrm{~m} \times 1.2 \mathrm{~m} \mathrm{x} 2.4 \mathrm{~m}$

Total $=27.4 \mathrm{~m} \times 1.2 \mathrm{~m} \times 5.0 \mathrm{~m}$

$\left(90^{\prime} \times 4^{\prime} \times 16.5^{\prime}\right)$

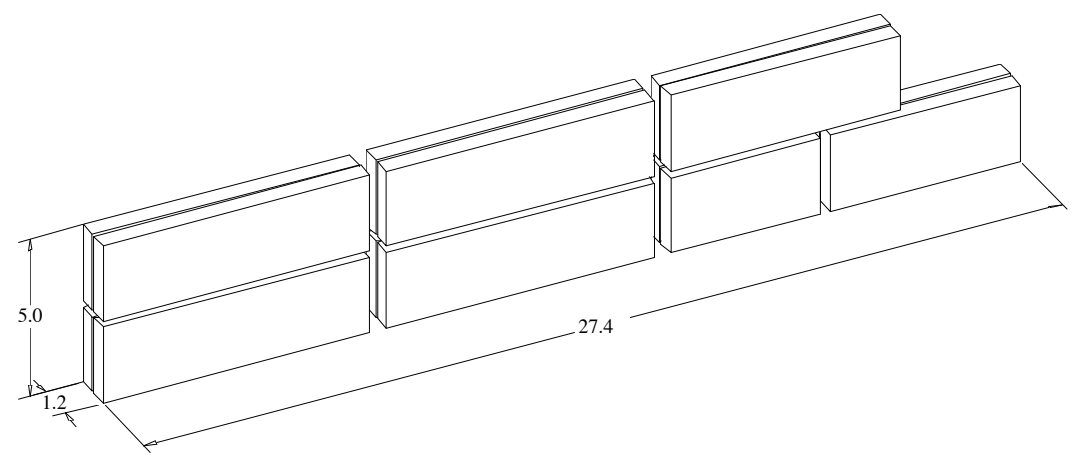

Figure 29. Extrusion Storage Space- 3 month supply

\section{Scintillator Storage}

222 gals required per plane - stored in 55 gal barrels (refer to Fig. 30)
a) $.56 \mathrm{~m}$ dia. per barrel
b) $.92 \mathrm{~m}$ high $+.14 \mathrm{~m}$ for pallet
c) 4.036 - 55 gal barrels required per plane $(.56 \mathrm{~m} \times .92 \mathrm{~m})$
d) $120 * 4.033=484$ barrels

Stack barrels 4 high and 4 wide

$484 / 4^{2}=30.25$ barrels long

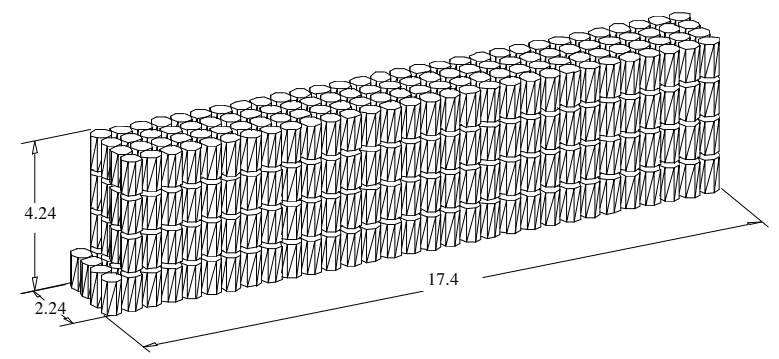

$$
\begin{aligned}
& 31 * .56=17.36 \mathrm{~m} \text { long } \\
& 4 * .56=2.24 \mathrm{~m} \text { wide } \\
& 4 *(.92+.14)=4.24 \mathrm{~m} \text { high }
\end{aligned}
$$

Figure 30. Barrels of Scintillator-3 months supply

Scintillator Storage $=17.4 \mathrm{~m} \times 2.24 \mathrm{~m} \times 4.24 \mathrm{~m}$ $\left(57^{\prime} \times 7.4^{\prime} \times 14^{\prime}\right)$ 


\subsection{APPENDIX E}

\section{COSTING OF LIQUID SCINTILLATOR DETECTOR}

Approximate costing summary of a liquid scintillator detector is shown in Table 4. The costs are obtained from a detailed costing spreadsheet for each item in the second column. The largest cost item is the active detector layer. The major cost items in the active detector are $\$ 4.5 \mathrm{M}$ for photo-detectors, $\$ 1.5 \mathrm{M}$ for plastic extrusions, $\$ 2.0 \mathrm{M}$ for optical fibers, and $\$ 2.0 \mathrm{M}$ for liquid scintillator. Just these four items account for $65 \%$ of the total active detector costs.

\begin{tabular}{|l|l|rr|}
\hline & \multicolumn{2}{|c|}{ MINOS Cost Summary } \\
\hline WBS & \multicolumn{3}{|c|}{ Total Cost: Base Year Dollars } \\
& & & 1996 \\
& & $\$$ & $4,691,477$ \\
1.1 & Excavation \& Hall Prep & $\$$ & $6,742,626$ \\
1.2 & Far Detector Systems & $\$$ & $2,169,463$ \\
1.3 & Near Detector Systems & $\$$ & $8,982,416$ \\
1.4 & Far Detector Structures & $\$$ & $1,815,301$ \\
1.5 & Near Detector Structures & $\$$ & $15,097,980$ \\
1.6 & Active Detector Elements & $\$$ & $5,567,943$ \\
1.7 & Electronics & $\$$ & $1,303,714$ \\
1.8 & Detector R\&D & $\$$ & $1,083,693$ \\
1.9 & Soudan 2 & $\$$ & $47,454,613$ \\
& & $\$$
\end{tabular}

Table 4. Costing summary of a liquid scintillator detector.

Table 5 on the next page shows a cost summary of another type of detector based on Iarocci tubes or RPCs. The cost for this type of detector is less than a liquid scintillator detector, but the performance is not as broad as the liquid scintillator detector. Most of the reasons are related to detector response during different types of physics experiments. 
Some of the items in Table 5 have the same cost as the liquid scintillator detector. The largest cost difference is the active detector layer. The active detector layer in Table 5 is only $\$ 8.66 \mathrm{M}$ versus $\$ 15.1 \mathrm{M}$ for the liquid scintillator detector.

\begin{tabular}{|l|l|rr|}
\hline & \multicolumn{2}{|c|}{ MINOS Cost Summary } \\
\hline WBS & & Total Cost: Base Year Dollars \\
& & & 1996 \\
& & $\$$ & $4,691,477$ \\
1.1 & Excavation \& Hall Prep & $\$$ & $8,258,983$ \\
1.2 & Far Detector Systems & $\$$ & $2,169,463$ \\
1.3 & Near Detector Systems & $\$$ & $8,912,666$ \\
1.4 & Far Detector Structures & $\$$ & $1,815,301$ \\
1.5 & Near Detector Structures & $\$$ & $8,657,378$ \\
1.6 & Active Detector Elements & $\$$ & $6,316,329$ \\
1.7 & Electronics & $\$$ & $1,303,714$ \\
1.8 & Detector R\&D & $\$$ & $1,083,693$ \\
1.9 & Soudan 2 & $\$$ & $43,209,005$ \\
& & $\$$
\end{tabular}

Table 5. Costing summary of a detector based on Iarocci tubes or RPCs. 